\title{
STORE AND FORWARD ROUTING FOR SPARSE PICO-SATELLITE SENSOR NETWORKS WITH DATA-MULES
}

\author{
A Thesis \\ presented to \\ the Faculty of California Polytechnic State University, \\ San Luis Obispo
}

\author{
In Partial Fulfillment \\ of the Requirement for the Degree \\ Master of Science in Computer Science \\ by
}

Trevor Joseph Koritza

June 2009 
(c) 2009

Trevor Joseph Koritza

ALL RIGHTS RESERVED 
COMMITTEE MEMBERSHIP

TITLE:

STORE AND FORWARD ROUTING FOR SPARSE

PICO-SATELLITE SENSOR NETWORKS

WITH DATA-MULES

AUTHOR:

Trevor Joseph Koritza

DATE SUBMITTED: June 2009

COMMITTEE CHAIR: John Bellardo, Ph.D.

COMMITTEE MEMBER: Clark Turner, Ph.D.

COMMITTEE MEMBER: Michael Haungs, Ph.D. 


\section{Abstract \\ STORE AND FORWARD ROUTING FOR SPARSE PICO-SATELLITE SENSOR NETWORKS WITH DATA-MULES}

Trevor Joseph Koritza

Satellites are playing an increasingly important role in collecting scientific information, providing communication services, and revolutionizing navigation. Until recently satellites were large and very expensive, creating a high barrier to entry that only large corporations and government agencies could overcome. In the past few years the CubeSat project at California Polytechnic University in San Luis Obispo (Cal Poly) has worked to refine the design and launching of small, lightweight, and less expensive satellites called pico-satellites, opening space up to a wider audience. Now that Cal Poly has the launch logistics and hardware under control, a new problem has arisen. These pico-satellites are within communication range of a ground station only 40 minutes a day. This, combined with their 1200 bps communication speed, limits the usefulness of the satellite missions to those only transmitting small amounts of data back to Earth. This thesis proposes a novel protocol that allows a sparse network of pico-satellites to communicate among one another and to larger satellites called data mules, which relay the information back to the ground station at much higher speeds. The data mules are able to provide higher speeds because they are larger satellites with less power constraints. This protocol makes it possible for a pico-satellite to send more data over a given amount of time with less end-to-end delay. When every satellite has large amounts of data almost three times as much aggregate data can be sent through the network, and almost five times more data can be sent if only a single satellite has large amounts of data to send. The end-to-end delay is cut almost in half when sending $1 \mathrm{MB}$ of data per day per satellite and is decreased by a factor of at least three when sending large amounts of data from only one satellite. 


\section{ACKNOWLEDGMENTS}

I would like to first thank Dr. Bellardo for getting me involved in this project, helping me think over ideas related to the protocol, and especially for writing the simulator that was used to test the protocol. Without his help I wouldn't have been able to have so much success in completing the project on-time and with such good results. I would also like to thank Dr. Turner and Dr. Haungs for their input on the initial iterations of the protocol and the research that went into creating the protocol. Finally I would like to thank my parents for helping me pay for college, so I was able to focus more on my school work allowing me to complete my thesis, and for supporting me throughout my life. 


\section{Contents}

List of Tables $\quad$ ix

List of Figures $\quad$ x

1 Introduction 1

1.1 Satellites and Orbits . . . . . . . . . . . . . . . . . . . 1

1.1.1 Geosynchronous Orbits . . . . . . . . . . . . . . . . 1

1.1.2 Low Earth Orbit (LEO) . . . . . . . . . . . . . . . . . 2

1.1.3 Pico-satellites .................... 3

2 Related Work 5

2.1 Store and Forward . . . . . . . . . . . . . . . . 5

2.1.1 Store and Forward: The early years . . . . . . . . . . . . 5

2.1.2 Store and Forward: Today . . . . . . . . . . . . . . 6

2.2 Satellite Routing . . . . . . . . . . . . . . . . . . 7

2.2 .1 ATM ....................... 8

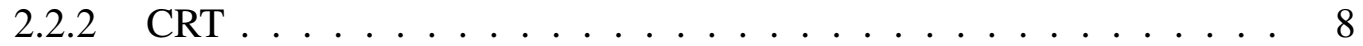

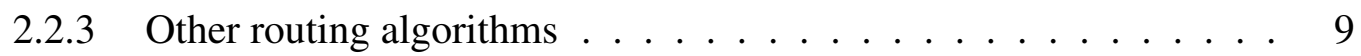

2.3 Hardware . . . . . . . . . . . . . . . . . . . 10

2.3 .1 IEEE $802.11 \ldots \ldots \ldots$

3 Motivating Application $\quad 11$

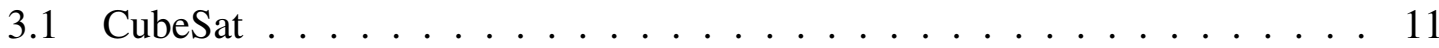

3.1 .1 PolySat . . . . . . . . . . . . . . 11

3.1 .2 GENSO Project . . . . . . . . . . . . . . . . 13

3.2 Sensor Networks . . . . . . . . . . . . . . . . . 13

4 Protocol Implementation 15

4.1 Data-link / Mac Layer . . . . . . . . . . . . . . . . . . . . 15

4.1.1 Types of Nodes . . . . . . . . . . . . . . . . . . . 15

4.1.2 Link State Tables . . . . . . . . . . . . . . . . 16

4.1.3 Satellite Connection States . . . . . . . . . . . . . . . . . 17

4.1.4 Duration Calculations . . . . . . . . . . . . . . . . . 19

4.1.5 Scheduling Connection Attempts . . . . . . . . . . . . . 19

4.1 .6 Mac Protocol . . . . . . . . . . . . . . . . . 21

4.2 Network / Transport Layers . . . . . . . . . . . . . . . . . . . . 23

4.2.1 Data Transfer . . . . . . . . . . . . . . . . 23

4.2.2 Command and Control Data . . . . . . . . . . . . . 24

4.2 .3 Shortest Path . . . . . . . . . . . . . . . . . 24

4.2 .4 Ford Fulkerson Algorithm [9] . . . . . . . . . . . . . . . . . 26

4.2.5 Implemented Shortest Path . . . . . . . . . . . . . . . . . . 28

4.2 .6 Forwarding Packets . . . . . . . . . . . . . . 32 
5 Protocol Evaluation 34

$5.1 \quad$ Simulator Implementation Details . . . . . . . . . . . . . . . . . . . 34

5.2 Comparison Protocol - Current PolySat . . . . . . . . . . . . 35

5.3 Evaluation Variables $\ldots \ldots \ldots \ldots \ldots$

6 Results 37

6.1 Goodput / Maximum Throughput . . . . . . . . . . . . . . . 37

6.1.1 Maximum data transfer with one satellite as the origin of all the data 38

6.1.2 Maximum data transfer when limited to a single hop . . . . . . . . 41

6.1.3 Maximum data transfer with multiple hops . . . . . . . . . . 43

6.2 End-to-end Delay . . . . . . . . . . . . . . . . . . . . 46

6.2.1 Sending 125MB file from CP4 to CP3 (data mule) . . . . . . . 46

6.2.2 Sending 250MB from MAST to CP3 (data mule) . . . . . . . 49

6.2 .3 Sending $1 \mathrm{MB}$ per day per satellite . . . . . . . . . . 52

6.3 Verification of Assumptions _ . . . . . . . . . . . . . . 55

6.3 .1 Data Mule Capacities . . . . . . . . . . . . . . . 56

6.3 .2 Predictability of Orbits . . . . . . . . . . . . 56

6.4 Correctness . . . . . . . . . . . . . . . . . . 58

6.4.1 Routing table being populated correctly . . . . . . . . . 58

6.4.2 Routing tables being used correctly to initiate connections . . . . . 59

6.4.3 Durations of connections are being calculated correctly . . . . . . . 59

6.4.4 Shortest Path is computed correctly . . . . . . . . . . . . . . 59

6.4.5 Data is transferred correctly . . . . . . . . . . . . . 60

6.5 Theoretical Results Using Ideal Connections . . . . . . . . . . . . . . 60

6.5 .1 Average Throughput . . . . . . . . . . . . . . 60

7 Future Work 63

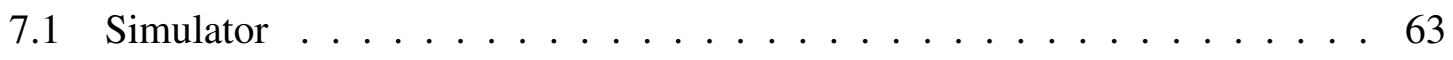

7.2 Protocol . . . . . . . . . . . . . . . . 63

8 Conclusion $\quad 65$

$\begin{array}{ll}\text { References } & 66\end{array}$

$\begin{array}{lr}\text { Appendices } & 69\end{array}$

A Packet Structures $\quad 69$

A.0.1 MAC Layer Header Packet . . . . . . . . . . . . . . . . . . . 69

A.0.2 ACK/NACK Packet. . . . . . . . . . . . . . . . . 71

A.0.3 Network Layer Header . . . . . . . . . . . . . . . . . . 72

A.0.4 Transport Layer Data Packet . . . . . . . . . . . . . . . . . 73

A.0.5 Transport Layer Instruction Packet . . . . . . . . . . . . . 73

B Example Simulation Output $\quad 74$

C Example Simulation Definition File $\quad 74$ 
D Header Files $\quad \mathbf{7 6}$

D.1 Satellite .......................... 76

D.2 Mac Layer . . . . . . . . . . . . . . . . . . . . . 80

D.3 Network Layer . . . . . . . . . . . . . . . . 82

$\begin{array}{llr}\text { E } & \text { Select Source Code } & 84\end{array}$

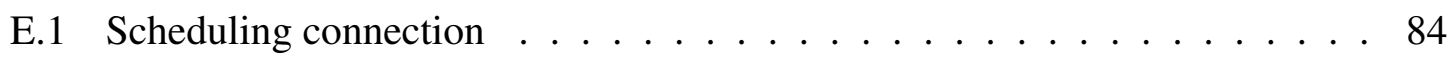

E.2 Shortest Path . . . . . . . . . . . . . . . . . 84 


\section{List of Tables}

1 Example of what a link state table looks like (SNSR is a Sensor) . . . . . . 29

2 Table depicting the pattern of sending a $1 \mathrm{MB}$ file per day directly to the ground station using a 1.2kbps link and assuming $40 \mathrm{~min} /$ day contact . . . . 54

3 MULE data rates required to not be a bottleneck, using 40 minutes per day exposure time ...................... 56

4 Actual Durations vs. Estimated Durations (SAUDI is short for SAUDI-

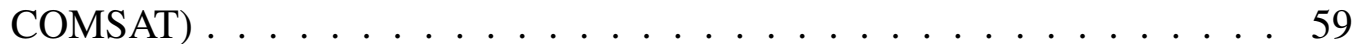




\section{List of Figures}

1 Satellite Orbit Types [21] . . . . . . . . . . . . . . . . . 2

2 CubeSat Satellite . . . . . . . . . . . . . . . . . . . . 12

$3 \quad$ P-POD . . . . . . . . . . . . . . . . . . 12

4 State Diagram for Satellite Connections . . . . . . . . . . . . 18

5 Example of using multiple passes as multiple nodes of a graph. . . . . . . 26

6 Example graph with capacities. . . . . . . . . . . . . . . . . . . 27

7 Initial capacity and flow. . . . . . . . . . . . . . . . 30

8 The flow after scheduling the sending of a 7 byte file. . . . . . . . 31

9 The flow after the first 3 bytes of an 18 byte file are scheduled. . . . . . . . 32

10 Final flow after scheduling both a 7 byte file and finishing scheduling an 18 byte file. . . . . . . . . . . . . . . . . . . 33

11 Maximum amount of data through network with only one satellite as the origin of the data. . . . . . . . . . . . . . . . . . . . . . . . 39

12 Collisions while sending the maximum amount of data through network from a single source. . . . . . . . . . . . . . . . . . 40

13 Maximum MBs transfered after a 365day simulation when sending data from every satellite to CP3 limited to a single hop . . . . . . . . . . . 42

14 Collisions after a 365day simulation when sending maximum data from every satellite to CP3 limited to a single hop . . . . . . . . . . . . . . 43

15 Distribution of data Sent after a 365day simulation when sending data from every satellite to CP3 limited to a single hop . . . . . . . . . . . . . 44

16 Maximum MBs transfered after a 365day simulation when sending data from every satellite to CP3 allowing multiple hops . . . . . . . . . . . 45

17 Collisions after a 365day simulation when sending maximum data from every satellite to CP3 allowing multiple hops _ . . . . . . . . . . . 46

18 Distribution of sent files after a 365day simulation when sending maximum data from every satellite to CP3 allowing multiple hops. . . . . . . . . . 47

19 End-to-end Delay of 125MB file from CP4 to CP3. (CP4 is the only satellite sending data) $\ldots \ldots \ldots \ldots \ldots \ldots \ldots$

20 Collisions while sending a $125 \mathrm{MB}$ file from $\mathrm{CP} 4$ to $\mathrm{CP} 3$. (CP4 is the only satellite sending data) . . . . . . . . . . . . . . . . . . 49

21 Number of sent and received packets while sending a 125MB file from CP4 to CP3. (CP4 is the only satellite sending data) . . . . . . . . . 50

22 End-to-end Delay of 250MB file from MAST to CP3. (MAST is the only satellite sending data) . . . . . . . . . . . . . . 51

23 Collisions while sending a 250MB file from MAST to CP3. (MAST is the only satellite sending data $\ldots \ldots \ldots \ldots$. . . . . . . . . . 52

24 Number of sent and received packets while sending a 250MB file from MAST to CP3. (MAST is the only satellite sending data) . . . . . . . . 53

25 Average Delays Sending $1 \mathrm{MB}$ per day from every satellite to CP3 . . . . 54

26 Collisions while sending $1 \mathrm{MB}$ per day from every satellite to CP3 . . . . 55

27 Time between accesses over 5 years CP3 to MAST (CubeSat Satellites) . . 57 
28 Time between accesses over 5 years CP4 to SAUDICOMSAT_6 (CubeSat Satellites) . . . . . . . . . . . . . . . . . 58

29 Average throughput over one year with one MULE vs max range of satellites for different bit-rates . . . . . . . . . . . . . . . . 61

30 Average throughput over one year vs number of mules at $150 \mathrm{~km}$ and $1 \mathrm{Mbps}$ bit-rate . . . . . . . . . . . . . . . . . . 6 62

31 Mac Header Packet . . . . . . . . . . . . . . . . . . . . . 69

32 Neighbor Entry . . . . . . . . . . . . . . . . . . . . . . 70

33 Acknowledgment Packet . . . . . . . . . . . . . . . 71

34 Network Layer Header . . . . . . . . . . . . . . . . . . . . . . 72

35 Data Packet . . . . . . . . . . . . . . . . . . . . 73

36 Instruction Packet . . . . . . . . . . . . . . . . . . . 73 


\section{Introduction}

Satellites are becoming more common for more and more applications including: connecting the most remote regions of the world to digital communications networks, positioning systems, military applications and scientific research. For the most part the satellites used for these applications are very large and expensive, not only to build, but also to launch. Projects such as CubeSat [20] have begun to explore cheaper solutions to putting satellites into space at very low orbits. These satellites called pico-satellites are small and lightweight with limited power and transmission capabilities, which is why very low orbits are used. These low orbits allow the satellites to need shorter range radios, but pose a few additional problems. There is a very limited exposure time when communicating with the ground station. Each pico-satellite is only in range of the ground station for approximately 40 minutes a day and only uses a 1200 bps connection. Existing techniques for connecting satellites do not work for this application due to the extremely low orbits, the fact that the network is not fully connected and the extreme degree of sparseness. My thesis will handle these problems by defining a novel protocol for communicating among a very sparse network of very low orbit pico-satellites. This is accomplished through using the satellites predictable orbits to compute shortest paths, store and forward techniques to transfer information between satellites, and with the use of sensor network ideas such as data mules.

\subsection{Satellites and Orbits}

\subsubsection{Geosynchronous Orbits}

Early satellite systems were designed for a low number of users and high bandwidth. In order to accomplish this Geosynchronous orbits (GEO) were used. GEO satellites orbit at an altitude of approximately $36,000 \mathrm{~km}$ over the equator and, from the perspective of an individual on the Earth, they do not move [8]. These satellites provide a constant service

area, making these early systems stationary repeaters as they forwarded a message from 




Figure 1: Satellite Orbit Types [21]

one ground station to another that were not in direct communication range. These satellites provide high bandwidth connections, however the latencies are very large, around $0.25 \mathrm{~s}$, as a result of the long distances the signals need to travel as well as the interference during those long distances. These large latencies can cause problems in many protocols and realtime streaming of voice and video [8]. Because the maximum data rate of the satellite is inversely related to the altitude of the satellite, putting satellites in lower orbits than GEO would cause not only lower latencies, but also higher data rates.

\subsubsection{Low Earth Orbit (LEO)}

LEO satellites orbit the earth at an elevation of $700 \mathrm{~km}$ to $1600 \mathrm{~km}$. LEO are generally polar orbits, an orbit that goes near both poles of the Earth. If an orbits altitude is less than $700 \mathrm{~km}$ (sub-LEO) rapid deterioration of the orbit occurs, the satellite loses altitude until it burns up during reentry. Along with a deteriorating orbit "maintain[ing] adequate satellite visibility" is also less of a problem with LEO orbits over sub-LEO orbits [11]. When it is in such a low orbit it will go under the horizon in relation to another satellite or a ground station sooner, losing the line of sight required to communicate. Polar orbits are beneficial because, by going over the north and south poles as the earth rotates, the satellite covers a lot of the Earth's surface. This includes remote areas where there may be a need for digital 
communication, but not enough to warrant putting up a GEO satellite.

The low orbit is the biggest advantage of LEO satellites. The satellite is not required to transmit information as far as GEO, allowing the satellite to use smaller antennas, less power, smaller batteries, and less surface area for solar panels. Decreasing these attributes greatly decreases the cost of the satellites hardware, building the satellite, and launching the satellite. The launching cost of a satellite is directly related to the weight and size of the satellite. The lower orbits also decrease the amount of radiation interference, especially due to the Van Allen Belt at an altitude of 1500km [11]. The most beneficial aspect of having a low orbit is the large decrease in latency when communicating with ground stations. LEO satellites have a latency of $0.125 \mathrm{~s}$, compared to the $0.25 \mathrm{~s}$ latency of a typical GEO satellite. With this in mind current researchers have focused on using LEO satellites to provide high speed Internet connections in mobile devices and digital communications in remote areas [8].

\subsubsection{Pico-satellites}

Most of what has been discussed so far is generic properties of either GEO or LEO satellites. This work, however, will focus on satellites with lower orbits than even LEOs. These satellites are pico-satellites most commonly related to the CubeSat program, described in section 3.1. CubeSats orbit the earth between $500 \mathrm{~km}$ and $700 \mathrm{~km}$ causing faster orbital deterioration than LEO satellites. The fast deterioration rate caused many studies to disregard these orbits as useful [4]. There are complex problems inherent in these orbits such as the difficulty of maintaining visibility with a ground stations and when communicating directly between satellites. These challenges include going in and out of range of each other frequently, for short periods of time, during what would otherwise be seen as uninterrupted communication. These short periods of time are called grey periods. These larger periods of inter-satellite connectivity, termed "connections", occur predictably. The grey periods are less predictable making it is necessary for the protocol be tolerant of short losses in 
connection. Pico-satellites have more stringent power constraints because of their small form factor. This results in less surface area for solar panels and less space for batteries further constraining their capabilities, therefore an energy efficient protocol is needed. 


\section{Related Work}

\subsection{Store and Forward}

Store and Forward is the act of buffering a message in the middle of a network for an unknown period of time until delivery to the final destination is possible. it is commonly used in networks with transient connectivity and reasonable expectation of eventual delivery. For example, when a link is temporarily unavailable due to it being out of range, in the case of a wireless network. However there are also other benefits gained from storing a message before forwarding it. One such example is time-shifting, storing a non-critical message until off peak communication rates are in effect.

\subsubsection{Store and Forward: The early years}

Store and Forward was first created in the early 1970's in order to handle the low bandwidth network connections and unsophisticated receive buffers. In these systems it was common for the receive queue to be full before all of the information was received from the sender. Because of the periods of time that the receiving machine could take to process the incoming information the sender would often times need to hold onto the information for some period of time before being able to forward information on. This problem was further complicated by the fact that the network connections often failed for substantial periods of time for various reasons. Computer scientists therefore came up with Store and Forward techniques to send information between nodes where intermediate nodes may need to hold onto the information for an unknown period of time before forwarding the information on [5].

With the introduction of current networking hardware and protocols store and forward techniques were no longer needed. Current networks are more sophisticated and reliable making store and forward techniques no longer needed. It wasn't until the mid 1980's

and the early 1990's that Store and Forward was once again needed due to the increased 
popularity of the microprocessor and micro-satellites [26, 4]. Micro-satellites were used to give developing countries digital communication channels. This was accomplished by relaying messages through Low Earth Orbit satellites (LEO), instead of through a terrestrial medium. A ground station is a stationary terrestrial based receiver whose purpose is to receive and send messages to satellites and keep track of the satellites. Ground stations can be anything from a single person with a radio receiver that is aimed at a satellite to a government run agency that keeps track of hundreds or thousands of satellites.

\subsubsection{Store and Forward: Today}

Store and Forward is still being used to connect ground stations using LEOs [12], but currently research is going into exploring Quality of Service (QoS), delay guarantees, and wireless Security.

Quality of Service (QoS) and Delay Guarantee - Quality of Service is an important aspect of any communication channel, especially one that can be used for future Internet and voice traffic. End-to-end delay is the QoS parameter that is focused on in both [14] and [13] because they claim it is one of the most important QoS parameters. End-to-end delay is the total time it takes between when the data is ready to be sent at the source to receiving the final byte of data at its destination. End-to-end delay guarantee research is looking into routing algorithms that take into account the available bandwidth of a link and how to fairly distribute that bandwidth among all of the satellites. This can either be a dynamic distribution of the bandwidth or can be a static distribution giving each connection equal amounts. No matter how the bandwidth is distributed the routing algorithm must be able to know when a link is congested and find the next best route to guarantee that the data will still be received within a specific delay bound [13, 14].

Security [3] - Due to the broadcast nature of satellite communications they are very vulnerable to attacks much like terrestrial wireless networks. Satellites however need be 
able to secure themselves without needing large amounts of time, computational power, or extra transmissions, in order to minimize power usage. Current research is going into efficient ways of securing satellite communication channels. Security is addressed by encrypting the information being sent, however, there are several different ways of encrypting that data. The most versatile approach is to give each satellite its own key and encrypting each packet for each individual receiver of that packet. This may be the most versatile approach, but it is extremely inefficient especially when packets are multicast. Therefore another approach is to use group keys. The difficulty with group keys is that the keys need to be updated every time a user joins or leaves the network. The approaches researched are similar to terrestrial based wireless networks, but the focus is on implementing them while staying within the power and computational constraints of the satellite. Security however is not a concern for my current work. Due to the short range nature of the communications that will be taking place a listener would have to be in space within range of the satellites transmitting, which is highly unlikely. Plus if the satellites in the network are amateur satellites, then they aren't allowed to encrypt their data.

Research is going into these areas so that LEO satellites can provide part of the infrastructure for the Internet and some voice applications. Both of these applications will be improved by obtaining better QoS [12]. The security aspect is not required for Internet use, but is desired for many other applications including government and commercial.

\subsection{Satellite Routing}

The most common protocol being used in inter-satellite communications is adapted from asynchronous transfer mode (ATM), however it is not perfect and research such as [28] describe better ways of implementing or replacing it. Many other routing algorithms have been studied and created focusing on different metrics and available information. 


\subsubsection{ATM}

ATM networks in satellite constellations has many advantages over terrestrial based ATM networks, such as no terrestrial disasters, able to cover a large amount of ground, and can be accessed with very small terminals [15]. The ATM satellite network described in [15] is based on one Geosynchronous satellite connecting several ground stations, but also discusses other more specific applications such as mobile communications channel using cellular technology. Their implementation shows that even though the MAC layer is not usually incorporated in ATM designs it is necessary in satellite communications due to the fact that satellite communication uses a shared medium. Several other ATM algorithms have been proposed for satellite communication, but most of them are not robust enough [28]. This is due to ATM generally using offline computation of shortest paths. When a packet needs to be sent the shortest path that is computed for that time period is broken or no longer exists then it takes time to recompute the shortest paths. By the time the newest path is found it may have already passed. Some solutions have several backup paths stored, but for networks that are highly dynamic these approaches do not work. [28] explores creating a robust on-line ATM-based routing protocol for use with a dense network of LEO satellites. The robustness of their system is tested compared to other ATM protocols using the sum of the amount of time that any particular satellite is unreachable.

\subsubsection{CRT}

Other routing algorithms have been proposed to create satellite specific protocols that take advantage of the specifics of satellite networks. CRT is an adaptive routing protocol that aims at providing routing to large constellations of LEO satellites. The main focus of this algorithm is to provide congestion control and load balancing over the constellation. However the protocol was designed to work with fairly complex hardware, requiring four transmitters and four receivers so each satellite can transmit and receive from all of its neighbors simultaneously. The interesting part of this protocol was its congestion control 
because weights are used to find out what the "best" route is from the source to destination. These weights are based on how much traffic is currently being transmitted by any particular satellite and is used during the computation of the shortest path. The weights are adjusted depending on how much data was sent during the last connection. [12]

\subsubsection{Other routing algorithms}

Enhanced Priority-based Routing (ePAR), is a priority based protocol that focuses on fully utilizing satellite links and not splitting flow between two paths when unnecessary. When splitting flows ePAR prioritizes the smaller flows to be moved to another link. The theory is that by moving the smaller flows less flows will need to be split between links and there will be less fluctuation in the flows of the routes. However, this routing algorithm was designed for a perfect dense mesh-like satellite constellation and the authors admitted that tailoring ePAR to a real satellite constellation would be very complicated. [17]

One final routing algorithm that is described in current research is using precomputed routes in satellite constellations. This routing protocol is not used for inter-satellite links (ISLs), but only for communicating from ground stations through satellites to other ground stations. Specifically this algorithm was designed to find the shortest end-to-end delay using different depth of hops. What was found in this study was that the more hops that were used the longer the computations took to find the shortest path, but also the end-toend delay decreased as well as the memory usage of individual satellites. This is because the packets are constantly moving from satellite to ground station back to satellite getting closer at each hop to the destination and staying in a satellites memory for a very short period of time between each hop. This study was an introductory study, so it doesn't have any insight on congestion control or real time adaptive routing, but contains good information on metrics for computing shortest paths for satellite routing. [10]

All of these projects deal with either inter-satellite communications focused on fully connected large meshes of satellites or deals with only communicating directly to ground 
stations. My work will focus on satellites that are very sparse, very rarely will these satellites be in range of one another and almost never will there be more than two in range at once. Because of the sparseness and discontinuity of the network, store and forward techniques need to be used not only in communicating with the ground stations as used in [10], but also in communicating between satellites like in [12] and [17]. Even though most of the related works focus on different types of satellites or ignore inter-satellite links, there are a lot of good techniques that can be learned from and used for smaller satellites in lower orbits.

\subsection{Hardware}

\subsubsection{IEEE 802.11}

[24] adapts the parameters of the IEEE 802.11 specification in order to provide intersatellite links using off the shelf IEEE 802.11 hardware. Starting at the MAC layer, the inter-frame timing needed to be redefined for inter-satellite links (ISL). IEEE 802.11 was designed for use in terrestrial applications where the maximum range is generally about 300 meters opposed to LEO satellites which have ISL ranges on the order of $5000 \mathrm{~km}$. Therefore the MAC timing must be increased from 10us to $34 \mathrm{~ms}$. This study also adapted other parameters such as changing the congestion window values from static to dynamic, so the protocol can adapt to changing conditions. The final aspect that was looked into in this study was using high-gain smart antennas to help with finding and tracking-over-time of all in range neighbor satellites and compared the range to the different types of antennas that could be used. With these adaptations this paper showed that IEEE 802.11 can be used for ISLs with minimal degradation of throughput. This information is valuable to show that hardware is available to use ISLs. 


\section{Motivating Application}

The motivation for using orbits lower than LEO satellites and using pico-satellites is provided through an ongoing project called CubeSat and its ground station counterpart PolySat. This section will discuss these projects and what problems this protocol will fix.

\subsection{CubeSat}

The CubeSat project is an "international collaboration of over 40 universities, high schools, and private firms" who's goal is to build, launch, and operate pico-satellites [20]. All of the information provided in this section, as well as figures were taken from the cubesat website provided by California Polytechnic University in San Luis Obispo, CA (CalPoly)[20]. The payloads of these satellites vary from private commercial payloads, scientific payloads and government payloads. The CubeSat, shown in figure 2 , is a $10 \mathrm{~cm}$ cube with a mass of no greater than $1 \mathrm{~kg}$. The focus of this project is collaboration between developers all over the world.

In order to launch the CubeSat satellites, CalPoly has designed a standard deployment system, the Poly Picosatellite Orbital Deployer (P-POD) (Figure 3. The P-POD is a tubular, spring loaded mechanism that takes up very little room in the launch vehicle, used to expel the satellites from the rocket once at the required altitude and protect the CubeSats from other payloads in the launch vehicle and visa-versa. Currently CubeSats are launched by decommissioned Russian rockets through companies such as Eurokot and Kosmotras, costing approximately $\$ 40,000$ per cube per launch for the developer.

\subsubsection{PolySat}

The CubeSat project takes care of the hardware of the P-POD and the specifications of the pico-satellite cube, but PolySat and projects like it around the world are in charge

of the internal payloads and the ground stations to speak with these satellites. PolySat 


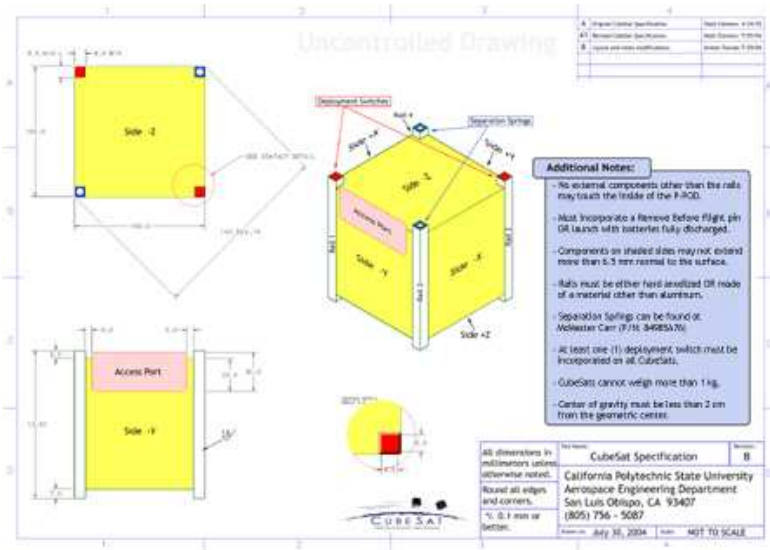

Figure 2: CubeSat Satellite

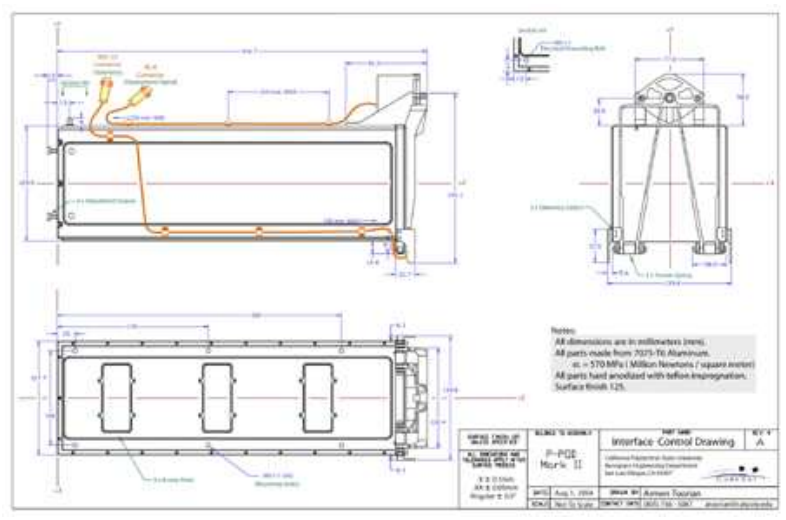

Figure 3: P-POD

is based at California Polytechnic University in San Luis Obispo, CA where a group of interdisciplinary students work to design, build, and operate CubeSats. The goal of this project is to build small satellites to perform various scientific research and exploration of space with a low cost satellite [7]. The problem PolySat currently has is they are only able to communicate with the satellites in orbit twice a day for about 20 minutes each time. On top of that the current transfer speed between the satellites and the ground station is approximately 1200 bits per second. Therefore transmitting a single high resolution image down to the ground station could take up to several weeks to completely finish. That is why this project is so important, it will help increase this throughput back to the ground station greatly. 


\subsubsection{GENSO Project}

The GENSO Project [22] is attempting to make an educational network of ground stations that all participate in the CubeSat project. This would connect all of the ground stations together so any CubeSat that followed the standard and had its ground station as part of the network would be able to connect to any of the other ground stations in the network. As said in the previous section there is very little time during a day when communication can be made with a satellite, but if the satellites are able to be reached from any ground station it could allow nearly constant contact with any of the CubeSat satellites. The GENSO project would compliment this work allowing more connection time for data mules and sensors to ground stations. Using my protocol on the space end of GENSO could use the same data mules for multiple schools giving more reason for multiple data mules to be used, which could then talk to any of the ground stations in the network increasing the throughput even more.

\subsection{Sensor Networks}

The size and cost of the pico-satellites described in the CubeSat and PolySat programs make them ideal for creating a sensor network in space. A sensor network can be used in several applications such as environmental monitoring, wildlife monitoring, or any other kind of scientific measurement. The purpose of these nodes is to gather data from their sensors then transmit the data back to a sink. The sink is simply the location where the data is collected together for future analysis, which would be the ground station in a satellite network. The sensor nodes can be stationary nodes, such as a sensor attached to a street light or tree, or can be mobile, such as a sensor attached to an animal or a bus [16].

There are different ways of getting this data from the nodes back to the sink. A multihop route can be computed to send the data from sensor to sensor until it gets to the sink, the sensor can wait for the data to manually be retrieved, or a data mule can be used. A data mule is a larger node that has fewer power constraints, can usually store more information, 
and can transmit longer distances. The data mules are usually mobile nodes that don't collect any information themselves, but are there only to relay the information from other nodes back to the sink [23]. In [27] a submarine robot was used as a data mule to retrieve data from mobile sensors on the ocean floor that were collecting information on marine microorganisms. The power constraints on the sensor nodes make it difficult to use them to transmit the data back to the sink because there will generally be only a few nodes that will need to relay a large portion of the traffic (the nodes closest to the sink). This is why data mules are so useful. They don't have the power constraints that the sensor nodes do, plus they generally can store more information so there is less risk of overloading the mule.

Some interesting uses of sensor networks have involved wildlife tracking, such as in [16]. This paper described ZebraNet a wildlife tracking project where electronic collars are carried by animals in a large wildlife area. The collars act as a peer to peer network to deliver logged data back to researchers. The data collected includes position, speed, temperature, weather, and environmental information. One of the large difficulties of this system is that the nodes are moving in a pseudo random fashion and the sink, which is the researchers camp, is also moving. Therefore these nodes use GPS to track where the animals are in relation to the researchers to get the information back.

Looking back at the CubeSat project it is apparent that it can be viewed as a sensor network in space. As of now all the sensors talk directly to the sink, but my work will show that more throughput will be achieved by allowing the pico-satellites to be able to transmit data among one another and to a data mule. 


\section{Protocol Implementation}

The basic implementation of the protocol is split into a few loosely coupled layers: the data-link / mac-layer, the network layer, and a minimal transport layer. The layers are not completely uncoupled in order to maintain efficiency which may be lost in completely separating the layers.

\subsection{Data-link / Mac Layer}

The Data-link layer is in charge of gaining the required information to know approximately when any satellite will be in range of any other satellite and find shortest paths, maintaining state information during a connection, dealing with collisions, dropped packets, timeouts, acknowledgments, and scheduling the sending of packets to initiate a connection.

\subsubsection{Types of Nodes}

Every node in the network is designated a type in order to know what their purpose is in the network. The types that are currently implemented are Low Latency Mule (LLM), High Bandwidth Mule (HBM), and Sensor. A LLM's purpose is to relay small amounts of information between the Sink and the sensors with a low latency but will have very little available bandwidth. This will mainly be used for instructions to be sent to the sensors as well as link state information. This is going to be a node with a short period, allowing it to talk to each node more often than the HBM. In the case of the CubeSat project the ground station, which is also the Sink, will act as a LLM, allowing the Sink to communicate to each satellite for a few minutes a day. The HBM is the data mule, a higher powered and larger satellite in charge of transmitting large quantities of data, like images or complex measurements, from sensors down to the Sink. The HBM has a longer period between passes with each satellite, but it will stay generally stay in range for longer durations. The HBM will also have a higher bandwidth connection with the sensors because the range will 
be smaller and have less atmospheric interference than from a sensor to the Sink. This will also save power by the sensor because each sensor will be sending the data a shorter distance with a lower transmit power. The HBM will then have to relay this information down to the Sink fast enough not to be a bottleneck in the system. Since the HBM is a larger satellite with more power and a larger antenna it will be able to transmit data down to the sink with higher rates than the sensors themselves can. In the case of the CubeSat project, the sensors see the Sink, or the ground station, 40 minutes a day, but will only see the HBM every one or two months. The final type of node is the sensor itself. The sensors can be gathering any sort of data from the payload to be transmitted to the Sink. Currently there is only one type of sensor, but there is room to add different types of sensors or sensor values with different priorities.

\subsubsection{Link State Tables}

In order to compute the shortest path and approximate when two satellites will pass each other some link state information must be collected. Link state tables are lists of information that describe the connectivity of a network. Because this network is not fully connected the link state tables in this protocol have additional information giving not only what two satellites connect, but also when they connect and for how long. Each satellite has a unique hardware Address that must be known to differentiate between satellites and to know what satellite a connection is related to. The types of both of the satellites must be known so routing to both is possible depending on the data that needs to be delivered. This allows the satellites to differentiate between sensors, LBM, and HBM so data can be sent through the most appropriate channel. The time last seen is the time that the satellites last became within range of one another and started to communicate and is used for calculating when the satellites will be in range again. The duration is used in conjunction with the bit rate to calculate the amount of data that could be transferred during a connection if necessary. The duration isn't simply saved as the time from the first contact to the time the satellites lose 
contact in the end. Instead the duration removes grey periods and takes into account the number of packets that are lost during the last transmission. This provides a slightly better estimate of how much data will be able to go through during the connection. The final piece of data is the time until the two satellites will be in range again. This in combination with the time last seen will allow a satellite to calculate when it will be in range again as well as compute the shortest paths. The link state information required in order for the power save mode to work is as follows: satellite 1 ID, satellite 2 ID, satellite 1 type, satellite 2 type, time of last connection, duration of last connection, bit rate of connection, and the approximate period between connections. This information is necessary to compute shortest paths, along with knowing approximately when to attempt to initiate communications with a specific satellite.

Each satellite will keep its own link state table that contains all the information necessary to know when each pair of satellites will be in range of one another. Every satellite will contain a special entry in the 0 index of the table that contains the information about itself. This entry is useful to simplify the sending of neighbor entries as well as sending its own type information and when received by another satellite it is made obvious what satellite is being communicated with. With this implementation the first entry in the routing packet will be about the satellite that is sending the information, by having the satellite IDs be equal the receiving satellite knows who it is talking to and its type. That satellite can then get the time last seen information from its own system clock and compute the estimated time until next pass. The link state tables get updated during the data exchange which is defined by the state diagram below.

\subsubsection{Satellite Connection States}

When going through a connection between two satellites each satellite has several states it needs to go through in order to complete the connection and pass all the required data. The state information serve many purposes, first of all, it allows the satellite to keep track 


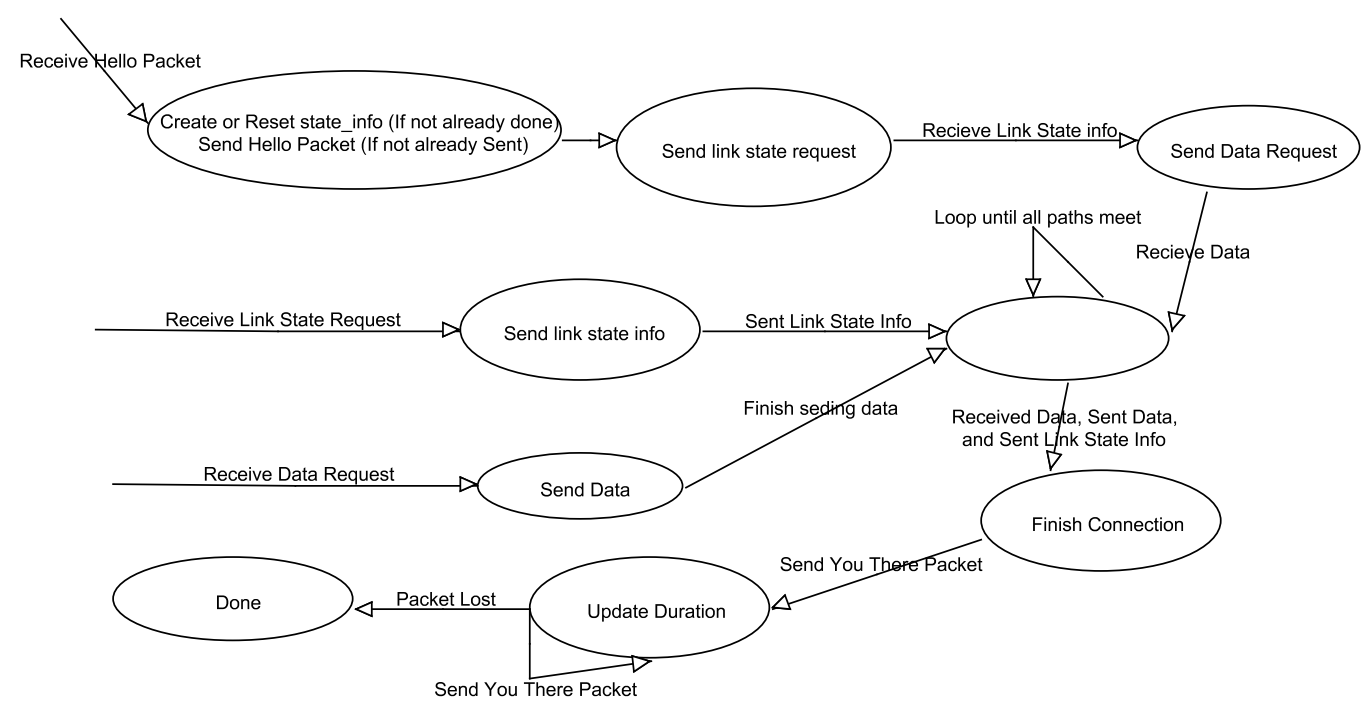

Figure 4: State Diagram for Satellite Connections

of a connection between two satellites. This is complicated by the fact that the satellites will go in and out of range during a single "connection" due to the non-parallel nature of the orbits, which were referred to as grey periods in the introduction. The states keep track of the connection and makes sure that it can differentiate between a grey period and a new connection to that satellite. The states also make sure that the minimum amount of information is sent between the satellites during every connection. This information is the updated link state information for other satellites in the network. The states can be extended in the future to contain other state information for a single connection like more advanced rate of change for the time between connections, or information tracking missed connections due to a temporary problem or a bad estimation of position. The states can also be thought of as an ordered checklist that needs to be completed before deciding the connection is complete. When the satellite reaches the final state that shows that a connection has completed the sending of all necessary information the node with the smaller ID of the two begins sending "you there?" packets. These packets are analogous to two people standing in a dark room and one of them continuously asking at some interval "Are you still there?" and the other one responding in the affirmative. This is part of keeping track of the duration of the connections, the next section will go into more detail on the calculation of durations. 


\subsubsection{Duration Calculations}

Grey periods make it difficult to calculate the actual duration of a pass between two satellites because they create holes in the connection. This makes it so it is not possible to simply take the difference between the time the connection started and time the connection ended to find the duration. Instead my algorithm keeps track of the last time a packet was received by the other satellite, including acknowledgments. If the time between packets is greater than five minutes then the algorithm assumes a grey period occurred and keeps track of the total time taken by grey periods during the pass. Every two hours the satellites check to see when the last time a packet was received, if no packet has been received in the past two hours then the connection is ended and the duration is calculated by the equation 1 where $T_{L}$ is the time the last packet was received from the satellite, $T_{S}$ is the time when the pass started, and $T_{G}$ is the amount of time taken out for grey periods. Two hours was chosen based on the observation that no grey period lasts this long, so if two hours passes without receiving a packet the satellite knows that the connection is over.

$$
\text { Duration }=T_{L}-T_{S}-T_{G}
$$

\subsubsection{Scheduling Connection Attempts}

Discovery Mode When a satellite is launched into orbit it has no information on how many satellites, or when it will encounter any satellites. Before it is able to schedule connections to other satellites it must find them and know when to expect a pass. The satellite learns this information by starting out in "Discovery Mode". In this mode the satellite sends out a broadcast HELLO packet every 60 seconds until it obtains enough information from each satellite to know approximately when it will be in range again. To know when two satellites will be in range they need to have passed one another twice. Two passes are required in order to calculate the approximate time between passes. Because this protocol depends on using a data mule, at least one HBM must be found before a satellite can 
leave discovery mode. Once the appropriate link state information is obtained and at least one HBM has been discovered the satellite can transition out of Discovery Mode and enter Power-save Mode. The time between passes of satellite can be anywhere from one month to several months, which means a satellite cannot leave discovery mode for at least this period of time. While in discovery mode the satellite also does not have enough information to find shortest paths, so no data can be sent until the satellite has left discovery mode. Not being able to transmit data for the first few months may not be acceptable, therefore this lengthy process can be eliminated by injecting link state information into a new satellite by the ground station. At the ground station the link state information can be calculated using the two line elements (TLE) of the satellites. The protocol is designed to not rely on the ground station to inject these links in order to allow the protocol to run without any outside help. This is beneficial if a scenario arises where there is no ground station to provide this information, such as if the satellites were orbiting a different planet.

Power-save Mode Once a satellite has left discovery mode it enters power save mode. Broadcast packets are no longer sent during power save mode. HELLO packets are sent only when the satellite expects it is about to pass another satellite. If a new satellite gets added to the network, in Discovery Mode, the other satellites in the network who are in Power-save Mode will still respond to the broadcast HELLO packets from that new satellite. This way the satellites saves power by not sending packets out when it doesn't think there is another satellite in range, but still is able to receive packets and respond to a new satellite adding the new satellite to its link state tables.

The HELLO packets are scheduled by using the information stored in the link state tables to estimate when two satellites will come into range again. Satellites must be able to schedule sending the Hello packet as to not miss the other satellite and needs to have a fail safe just in case the estimation is bad. Contained in the link state table is the information that describes the time between the last two passes, which can be used to estimate the 
time of the next pass. By taking the time last seen and adding to it the time between passes each satellite can estimate when the next pass will occur. However, if the next actual time between passes is larger or smaller a cushion or buffer to give a little leeway is included. To compensate for the time being smaller the satellite schedules the HELLO packet to be sent ten percent earlier than the time between passes that is stored in the link state table. For example, assume that the time last seen was at time 5 and the time between sightings is 20 . The time that the on event would be scheduled for would be $(5+20)-(0.1 * 20)=25-2=23$. As an added guarantee a minimum amount of time before the approximated connection is specified to make sure connections are not missed.

To deal with an estimation that is too early the HELLO packets are resent for longer periods of time than normal packets. The resend value is set to 20 percent of the time between passes from the link state table. Because the satellite is starting its attempt to connect 10 percent before its original time the satellite will give a 20 percent buffer so it can cover both the 10 percent before the original time and 10 percent over the original time. However if the next sighting is more than 10 percent off of the estimation either higher or lower it will miss the satellite and must have a way of recovering. If the HELLO packet is lost then the satellite needs to reschedule an the HELLO to be resent for the next connection. The time scheduled is for the same 10 percent buffer but for the time last seen plus the time between sightings times two. This is working under the assumption that the missed satellite was uncommonly different and will be closer to the estimation the next time. This was not encountered during any of the simulations run, but is a good failsafe to keep in the system.

\subsubsection{Mac Protocol}

The Mac sub-layer of the Data-Link layer is currently implemented as a basic Aloha. Aloha is a very naive mac protocol that simply sends data whenever data is available, then if a collision occurs it exponentially backs off to allow other packets to be sent. Even though 
Aloha has a maximum capacity of $18 \%$ [1] it is acceptable for this implementation because the mac protocol isn't the focus of this work and because this protocol generally has data only going in one direction it should get a better maximum capacity than this. Data only travels one direction because in this iteration data is only being sent from the satellites to the data mule, not the other way around. If it was desired to be able to upload data to the satellites then a more sophisticated protocol would be needed. In future iterations of this protocol the mac protocol may need to be updated, but for now Aloha is enough to get results.

Sending of Packets Packets to be sent are added to an outgoing queue for packets. Packets are generally added to the end of the queue, but can be added to the front in order to have expedited delivery of a packet. Acknowledgments and resent packets currently are added to the beginning of the queue instead of the end. The mac protocol will send packets up to a maximum window size. This window size is currently set to 20 , which means that a maximum of 20 packets can be sent before receiving acknowledgments for at least one of those packets. This number can be adjusted for specific transfer rates and to optimize the protocol, but through testing it was found that this was a reasonable size to use. Once an acknowledgments are received for one or more packets then the mac protocol will allow more packets to be sent until the maximum window size is reached again.

Acknowledgments Multiple acknowledgments are sent in a single packet in order to limit the amount of packets going between satellites, not only saving power, but also leaving more of the medium open for more data to be passed between the satellites. An ACK packet is sent immediately once the maximum window size, described in the previous section, of packets has been received, but there is also a maximum time between acknowledgments to allow for the possibility that the maximum window size is not reached. This maximum time is set to one fourth of the timeout time for a packet allowing ample time for the acknowledgments to be received so packets aren't unnecessarily resent. 


\subsection{Network / Transport Layers}

\subsubsection{Data Transfer}

Outgoing Data Queue In the transport layer each satellite that data can be sent to has its own outgoing data queue. The data that is stored in the queue is simply a group of packets that have already gone through routing process. Since each satellite passes each other satellite multiple times the satellite needs to know on which pass to transmit the data or a link will get congested. When two satellites first come into contact and start a new connection, the satellites will go through the outgoing data queue for that satellite and decrement all of the pass offsets. Then when the request to send data is received, the satellite looks through the queue for the requesting satellite and sends all the packets that have a pass offset of zero.

When data is provided to the transport layer from the payload of the satellite it is broken into packet size chunks, has the shortest path computed and placed in this queue. When the data request is received all packets with a pass offset of zero to that satellite will be transmitted. The receiving node will take the data packets in whatever order they arrive and once all packets for the data being processed have been received the receiving node can process it depending on the type of data.

If for some reason the estimated amount of data able to be sent is too high and the satellite queues too much data for the connection. The remaining data that was unable to be sent is removed from the outgoing packet queue and put back into the data queue corresponding to its next hop with an offset of one, to be sent out at the next connection. This should happen very rarely if the estimates are done properly, but it is a good failsafe in case unforeseen interference is experienced during a connection or if a satellite has a much shorter duration then estimated. 


\subsubsection{Command and Control Data}

Command and control packets are small amounts of data that generally fit into a single or very few packets. If the packet is time sensitive it will be sent through the LLM instead of the HBM so it will arrive at its destination sooner. Due to the fact that command and control data is limited to a single or very few packets, they do not have the bandwidth requirements that sending the data does. Therefore the satellite is able to send it directly to the LLM or the ground station instead of going through a Data Mule first. This will greatly reduce the latency of these packets and allow important command and control information to be received as soon as possible.

\subsubsection{Shortest Path}

Finding the shortest path from one satellite to another is much more complicated in sparse satellite networks than in most terrestrial or even dense satellite networks. In most terrestrial and dense satellite networks the network is fully connected so all that needs to be computed is which node is the next hop to get to the destination. This is especially straight forward if the network is static and doesn't change rapidly. Even when the network is dynamic if the network is fully connected finding the shortest path isn't terribly difficult, there are protocols such as AODV, DSDV, or DSR that are well known and researched that are currently used in fully connected mobile networks [6]. However in a sparse satellite network where the satellites are moving at different speeds the shortest path changes as a function of time depending not only where the satellite trying to find a shortest path is, but also where all of the other satellites in the network are located and the speeds they are moving.

Using the link state information a best first search algorithm is used in order to find the path to the destination where the delivery is the soonest. The interesting aspect of this algorithm will be that it can't treat each node as a single entity. Because the satellites are in orbit, each time two satellites pass each other is a new path that can be taken with a 
maximum load computed by the duration and bit rate of the connection.

Figure 5 shows an example of a three satellite network needing to find the shortest path from the source (S) to the destination (D) with one additional node (1). The vertices on this graph signify a pass between two satellites, for example the node labeled as 1, higher up on the graph, signifies the passing of the source satellite and satellite number 1 and the node labeled D that is just under this node signifies the passing of satellite number 1 and the destination. The edges on the graph have two characteristics that define them, first there is the time until the pass begins and secondly is the capacity of the link. Note this graph could be display with each satellite having its own vertex and multiple paths going between each vertex, but the graph shown in figure 5 is easier to see the progression of time. As the node gets farther away from the source the more time has gone by. The times shown are the times when the two satellites connected by the line will be in range of each other. As the graph shows there are three different routes to take from the source to get to the destination. The first route that is possible is sending the data directly to the destination on 12/02/2008 and again on 1/31/2009. The shortest route, however, is going through satellite 1 on 10/22/2008 which then relays the message on $11 / 14 / 2008$ to the final destination. This isn't the only route that is possible through satellite 1 . Due to the nature of orbits in approximately one month the source and satellite 1 will become in range again and a few months after that satellite 1 will be in range of the destination. The shortest path algorithm must also take into account the load on each of the edges. A modified version of the Ford Fulkerson flow algorithm can be used to find the available flow in combination with the shortest path to the destination. To find the maximum flow it is required that the flow information be shared among the satellites, but this may not be ideal because of the extra overhead as well as the infrequently that the satellites come into contact will make the information too old to be of use, so an estimation must me made. This estimation can be as simple as allowing any satellite to use the full capacity of any connection or giving each satellite a fraction of the link capacity depending on the total number of satellites. A more complicated estimation, 


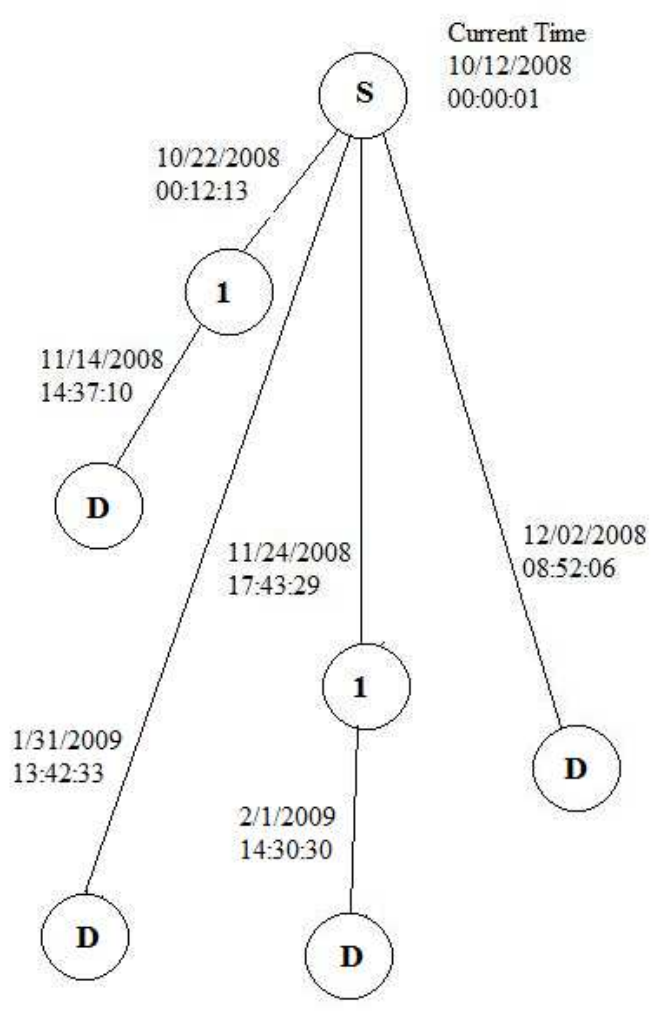

Figure 5: Example of using multiple passes as multiple nodes of a graph.

as in [12], can also be used where the capacity is dynamically changed depending on how much of the total capacity is required at each pass.

Figure 6 shows the same network as before, but includes example capacities for each link, these capacities are not real capacities they are used to simplify the explanation of the shortest path algorithm. Assuming the source has data of size 50 bytes, units aren't important for this example, to send to the destination it wouldn't be possible to send all 50 bytes along the shortest path. Therefore the data must be split along all four paths, which is where the Ford Fulkerson Algorithm comes in.

\subsubsection{Ford Fulkerson Algorithm [9]}

The Ford Fulkerson Algorithm is based on the Ford Fulkerson Method and is meant to find the maximum flow through a network. It is an iterative method that starts by setting all flow to zero then at each iteration finding an "augmenting path" from source to sink and in- 


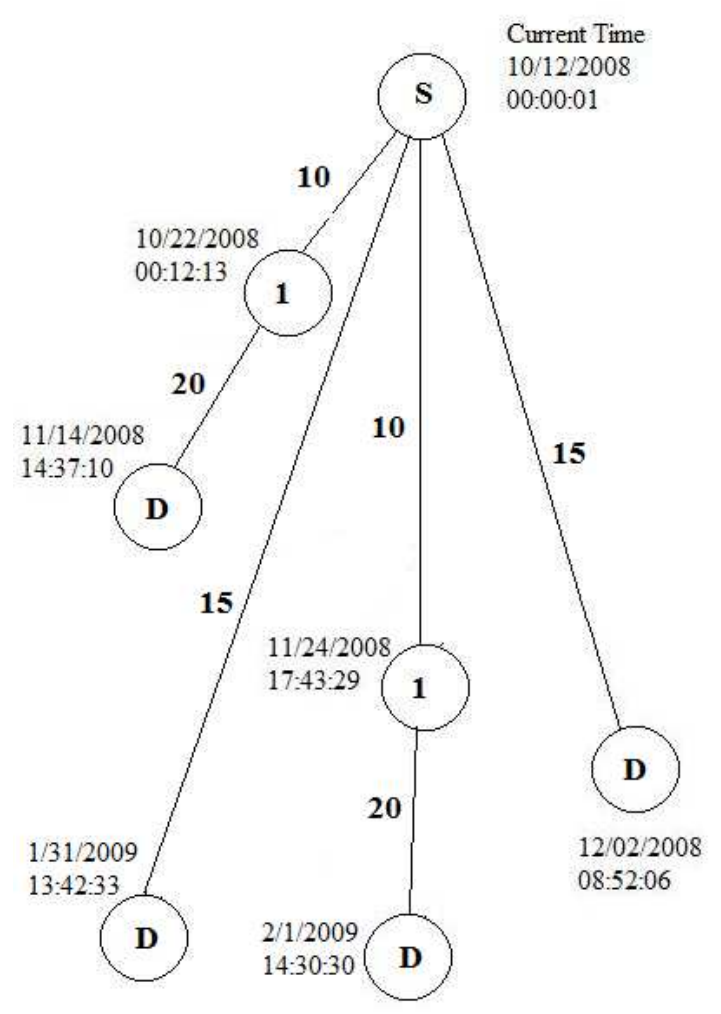

Figure 6: Example graph with capacities.

creasing the flow along that path. The maximum capacity of each edge on the graph minus the flow is the residual flow, or the available flow left in the network. An augmenting path is a path from source to sink in the residual network. Once there are no augmenting paths with a residual flow left the iterative process is over and the maximum flow is returned.

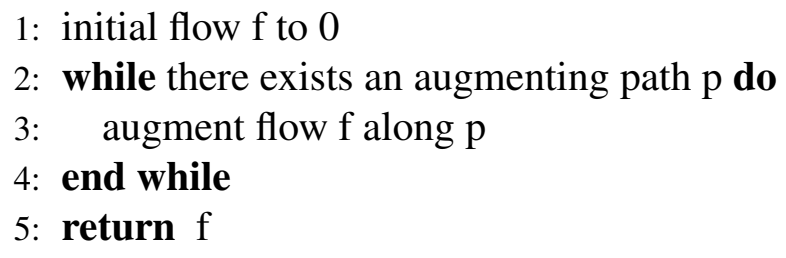

The Ford Fulkerson Algorithm builds on the Ford Fulkerson Method. The algorithm is expanded to show how to implement this in a network. First the algorithm sets the flow all to zero as stated in the method above. Then while there are still paths from the source to the sink in the residual network the algorithm finds the minimum residual capacity along 
each path. The for loop then increments the flow by that minimum value for each edge on the path. The last line of the for loop at first seems out of place, but its purpose is to make sure there is a maximum flow by setting the path from $v$ to $u$ to the negative of $u$ to $v$. This is to allow the algorithm to adjust the flow if the path algorithm it chooses first takes a path that may not be part of the optimal flow.

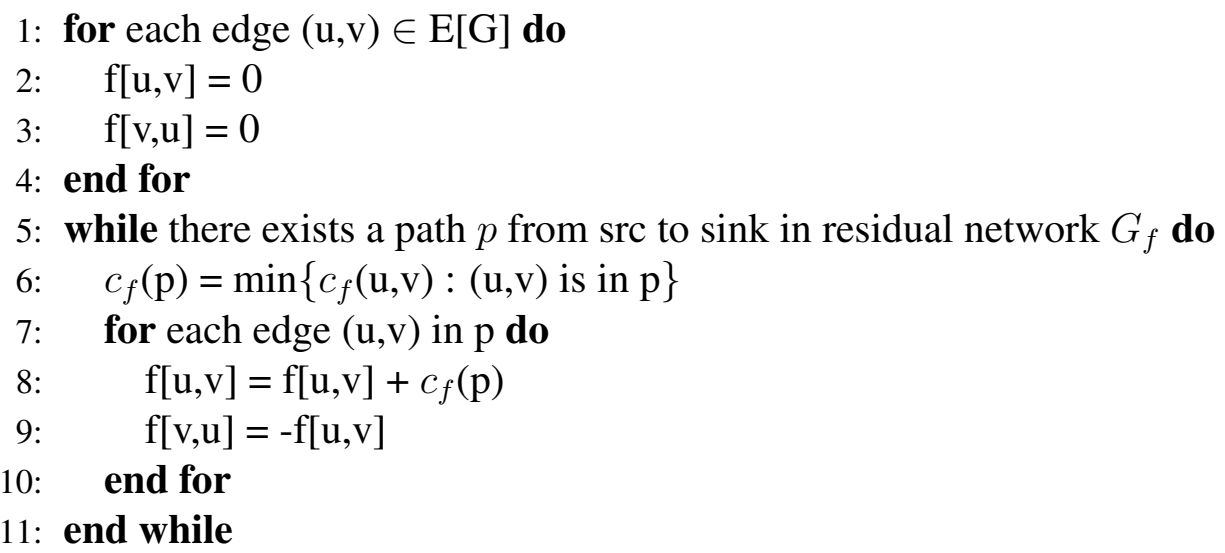

\subsubsection{Implemented Shortest Path}

The shortest path algorithm implemented is a modified version of the Ford Fulkerson algorithm. With the modifications it no longer holds the proof that it will always return the maximum flow, but still will in most cases. The loss of the maximum flow guarantee is due to not being able to compute the maximum flow throughout the entire graph. Calculating flow across every edge and vertex at once is not plausible also because the farther out the satellite looks the less accurate the estimates will be because the orbits do deteriorate and the periods change. On top of this the flow is not set to zero every time the algorithm is called and flow isn't ever moved from one link to another.

To implement this shortest path algorithm the maximum capacities of each edge on the graph need to be computed as being a fraction of the duration multiplied by the bit rate of the connection in order to not over estimate the size of the link and leave room for high priority instructions that may need to go through a link. When data becomes available to 


\begin{tabular}{|c|c|c|c|c|c|c|c|}
\hline ID1 & ID2 & Type1 & Type2 & Last Connection & Duration & Bit Rate & Time Between \\
\hline S & 1 & SNSR & SNSR & $9 / 18 / 2008$ & $2 \mathrm{~s}$ & 5 bytes/s & 34 days \\
\hline S & D & SNSR & HBM & $10 / 03 / 2008$ & $3 \mathrm{~s}$ & 5 bytes/s & 60 days \\
\hline 1 & D & SNSR & HBM & $8 / 30 / 2008$ & $4 \mathrm{~s}$ & 5 bytes/s & 76 days \\
\hline
\end{tabular}

Table 1: Example of what a link state table looks like (SNSR is a Sensor)

be sent to the sink its size plus the overhead due to packet structures is sent to the modified Ford Fulkerson algorithm. This algorithm follows the same process as the original Ford Fulkerson Algorithm, except that every time the algorithm is called it does not set the flow to zero. This is so when there are multiple files to send each one will modify the flow individually and will not need recompute the shortest paths and flows for every file that still needs to be sent.

The path through the residual network is computed by a best first algorithm based on time of delivery. This algorithm returns the lowest latency path that still has some residual flow available. If no residual flow is available on the shortest path then the next shortest is returned and so on. This path is returned back to the Ford Fulkerson algorithm which then is searched for the minimum flow available on any of the edges in the path, which is then used to increment the flow for each edge. Knowing what edge is being used is not enough in this network. It is also required to know which pass being referred to. Therefore an offset was put into both the shortest path and the flow to keep track of the flow available on each pass not just the next pass. For the actual code which implements this algorithm refer to the Appendix section E.2.

Figures 7 to 10 give an example of the algorithm in action and

In figure 7 the graph for the next few months is shown with the flow (all set to 0) and the max capacities of each link.

This example will start out with a 7 byte file being sent from the source $(\mathrm{S})$ to the destination (D). The first step is to find the shortest path, which is to go from the source to satellite 1 on $10 / 22 / 2008$ then to the destination on 11/14/2008. Now the algorithm calculates the how much to increment the flow for this iteration. Since the flow is 0 , this 


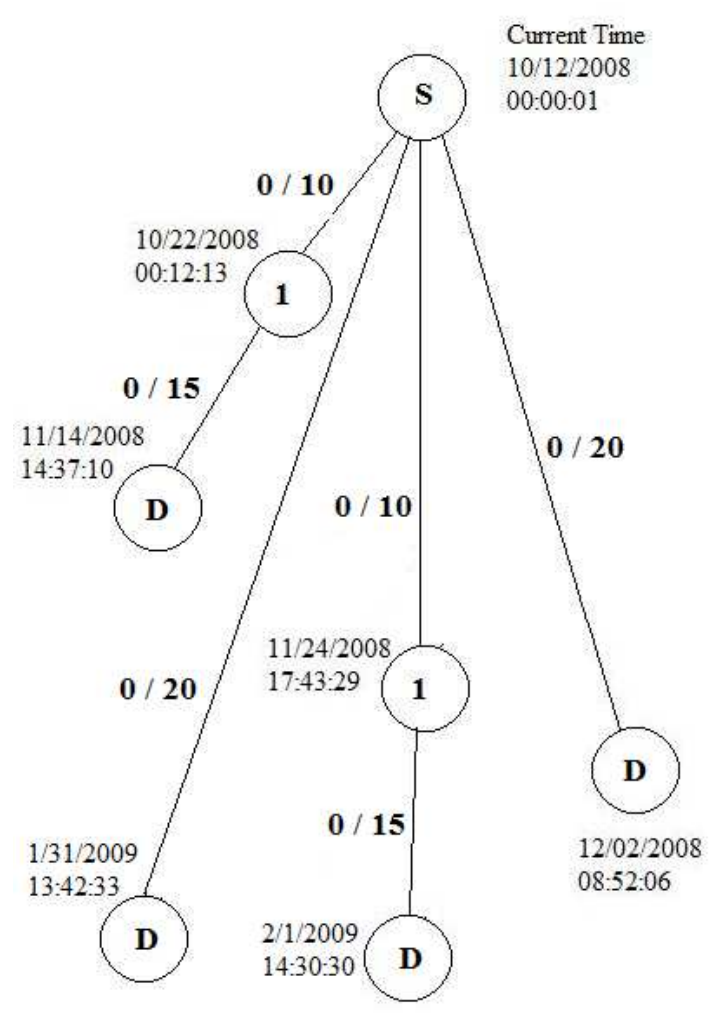

Figure 7: Initial capacity and flow.

being the first file sent, the residual flow is the same as the max capacity. Also because the size of the file being sent is less than the smallest capacity of the two links needed, the algorithm simply increments the flow along this path by the size of the file ( 7 bytes), which can be seen in figure 8 .

Now that the 7 byte file has been scheduled another 18 byte file needs to be sent. Once again the algorithm finds the shortest path that still has residual flow left. Again the shortest path is found to be the path through satellite 1 on 10/22/2008. This time however, because a file has already been sent the residual flow is only 3 bytes, so the algorithm increments the flow along this path by only 3 bytes as seen in figure 9 . This leaves 15 bytes left that haven't been sent yet, so the algorithm has to compute the shortest path again.

This time computing the shortest path the shortest path doesn't have any residual flow left because the residual flow between the source and satellite 1 is zero. Therefore the shortest path returns the shortest path that still has residual flow left. The path directly to 


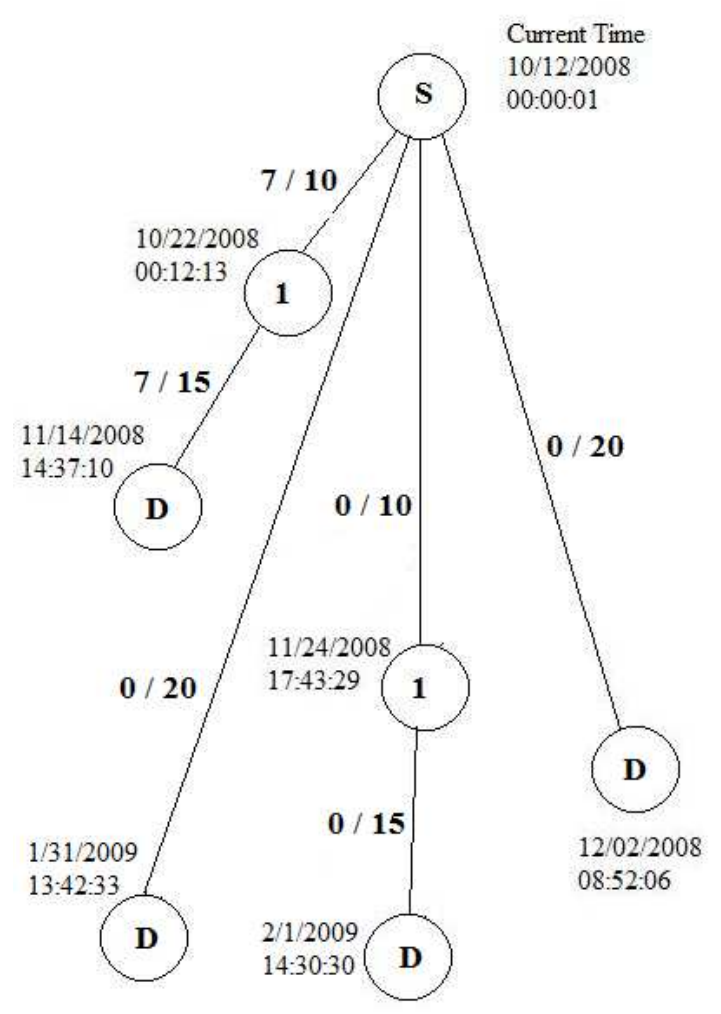

Figure 8: The flow after scheduling the sending of a 7 byte file.

the destination on 12/02/2008. Since there are only 15 bytes left to send of this file and the residual flow left on the link is 20 bytes, the flow is incremented by 15 bytes as shown in figure 10. This figure is the final product of sending two files, one of size 7 bytes and one of size 18 bytes through this example network. Notice that between the files the flow was not reset to zero and the algorithm does not go through and find the absolute maximum flow for the entire graph. These differences are done to deal with the fact that as time goes on more and more nodes are going to be added and the exact times and durations of the links can change and even though because of these changes the absolute maximum flow is not provably found, it is a close enough estimate that the majority of the time it is still the maximum flow.

The flow information is stored for each connection between every two satellites. Therefore the flow information is stored in an array of size NUMSATELLITES $\times$ NUMSATELLITES $\times$ MAXPASSOFFSET. 


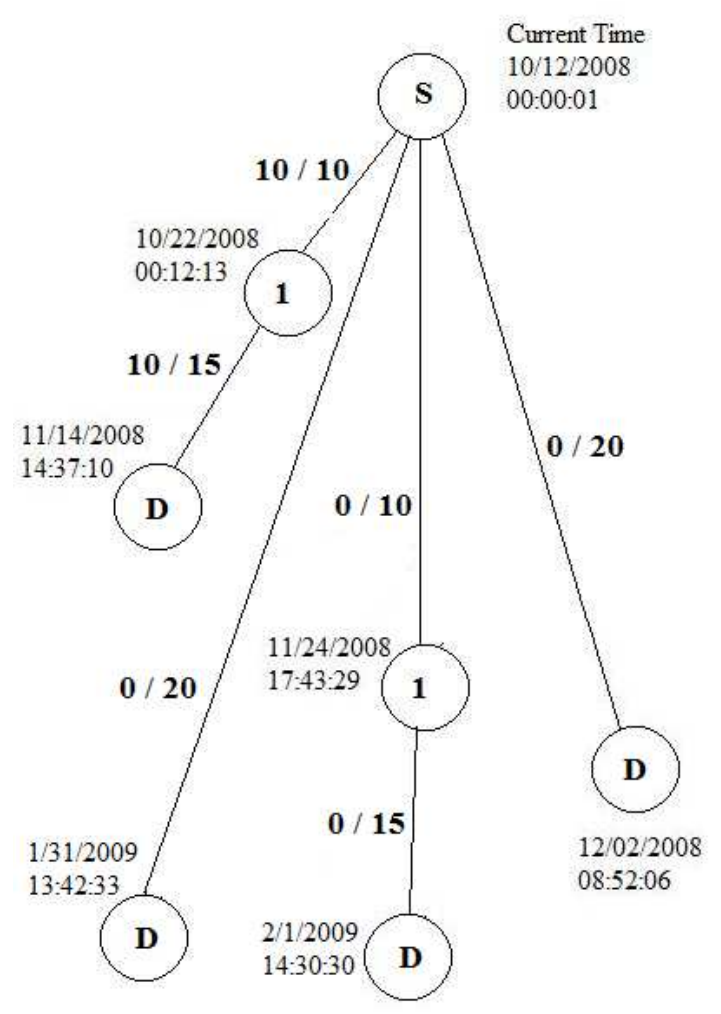

Figure 9: The flow after the first 3 bytes of an 18 byte file are scheduled.

NUMSATELLITES is simply the number of satellites that are in the current network and $M A X P A S S O F F S E T$ is the maximum number of passes in the future that have flow values greater than 0 . The $M A X P A S S O F F S E T$ can be increased if need be or the data being scheduled can wait until a few passes have completed. When two satellites start a connection this array is updated removing the flow from the current connection from the array. Once the connection has started this flow value is no longer needed because data can no longer be scheduled to be sent out on this connection, any new data to be sent out will have to wait for the next opportunity.

\subsubsection{Forwarding Packets}

When a packet is received whose final destination is not the current satellite, the packet must be forwarded on. The packet is put through the shortest path algorithm and added to the corresponding data queue for the next hop satellite. Potential problems can occur here 


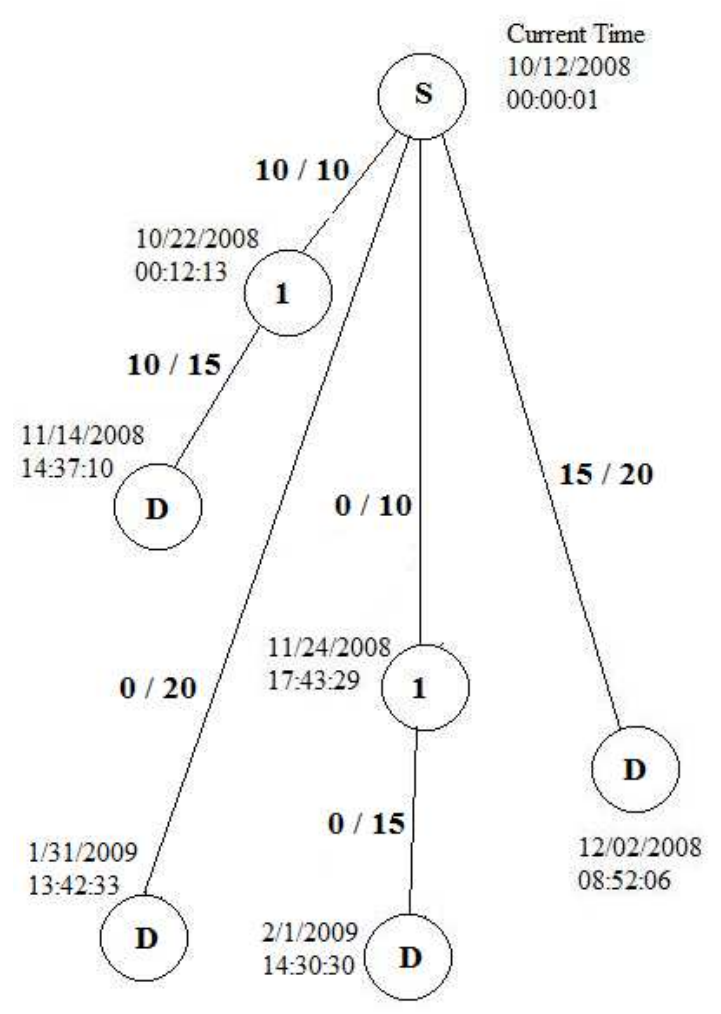

Figure 10: Final flow after scheduling both a 7 byte file and finishing scheduling an 18 byte file.

if the receiving satellite computes a different path than the source satellite did. The problem with this is the source satellite may have already sent enough data through that link that it is completely full. If the forwarding satellite sends more information through that node then the satellite may not be able to send all the data needed during the connection, delaying the delivery of the packets. This can be dealt with by either sharing the flow information between satellites along with the link state information or by performing source routing. Both of these techniques are out of the scope of this project and will be left for future versions of the protocol. 


\section{Protocol Evaluation}

There are several ways to go about evaluating the protocol, the satellite could be built, an emulator could be used, or a simulator. It isn't feasible to go about building the satellite and launching it in order to test the protocol until it is fully implemented and tested. It would be nearly impossible to debug the protocol once the satellite is in space and attempting test it while still on Earth wouldn't work because there wouldn't be a way of creating orbits. An emulator could also be used but because the hardware that is going to be used hasn't yet been decided the protocol needs to be tested without the hardware specified. Simulating the protocol is the best first step to evaluating and debugging it. By creating a simulator that uses the actual code that would be run on the satellite there will be little work left when it does come time to installing it on a satellite.

\subsection{Simulator Implementation Details}

For the simulator to be useful it must be able to simulate the three dimensional orbits of the CubeSat pico-satellites, detect collisions, and know whether or not two satellites are in range. More specifically it is important to simulate actual orbits rather than ideal orbits in order to make sure the protocol will work in a real life situation, such as handling grey periods.

A few of the simulators looked into were Network Simulator (ns-2), Satellite Tool Kit (STK) and creating a custom simulator using an open source two line element (TLE) calculator. NS-2 is a standard network simulator that is used for testing many different types of networks including terrestrial, satellites, and wireless networks [25]. The problem with NS-2 is the large learning curve making it unsuitable for such a short project and has many additional features that are unnecessary. Satellite Took Kit has an API that could be used to create a custom simulator and is used in industry, so would be a good choice, but it is expensive making it difficult to use for a project such as this and due to it being a com- 
mercial application not as much control is given over the source code limiting what can be accomplished by it [2]. The final choice is creating a custom simulator using open source software. This was what was decided upon because not only is the software free, but has just as accurate of results as a commercial program. PREDICT is one such open source project that is meant to track satellites in real time and can even interact with an antenna [18]. PREDICT is ideal because of its open source nature all of the source code is available to be tailored to becoming a simulator for the protocol and can gather whatever data needed. The simulator is a custom event driven simulator based on the PREDICT [18] software, modified by Dr. John Bellardo a professor at California Polytechnic State University in San Luis Obispo, CA. The simulator uses a priority queue of events that are sorted based on the time which the event occurs.

\subsection{Comparison Protocol - Current PolySat}

Currently, the PolySat program at California Polytechnic State University: San Luis Obispo maintains CP3 and CP4, two CubeSat satellites. These two satellites don't communicate with each other. Rather, CP3 and CP4 communicate only with the ground station located in San Luis Obispo when in range. This "competing protocol" inspired the development of this novel protocol. This novel protocol will be better than the current PolySat implementation in terms total throughput and end-to-end delay for large files, while still being comparable in end to end delay for small files.

\subsection{Evaluation Variables}

Simulations have been done varying the number of satellites, bit rate and maximum communication range. The number of sensor nodes from 1 to 4 and the number of mules nodes was kept at 1 for the simulations and varied in some feasibility studies. The maximum communication range varied from $50 \mathrm{~km}$ to $500 \mathrm{~km}$ and the bit rate was explored from 1.2 kbps to 50Mbps. These variables were used to compute theoretical maximum values for 
throughput to show what is required in order to be able to send more data then the current protocol described above. 


\section{Results}

The results show that this protocol shows substantial improvement over the currently used protocol by PolySat in both goodput and end-to-end delay. The results were gathered using one to five satellites. Additional data was gathered to compare the protocol to itself in different configurations and data loads in order to find where this protocol's strengths and weaknesses lie. The satellites used were from the Cubesat Project and were composed of CP3 and CP4 from Cal Poly [7], SAUDICOMSAT 4 and 6 commercial satellites from Saudi Arabia and MAST from Stanford University [19]. These satellites were all launched in 2007 and the TLEs being used are from 2009. The TLEs used for these satellites are from 2009, even though a TLE from just after launch would have supplied passes with longer durations. The reason for this was I was unable to find TLEs from 2007 or 2008 for SAUDICOM4 and SAUDICOM6, but according to the TLEs found for CP3, CP4 and MAST the durations were indeed longer just after launch. This will not pose a significant threat to this protocol however because if it is going to be in use for multiple years it will have to outperform the current implementation at two years and not just right after launch.

\subsection{Goodput / Maximum Throughput}

The goodput or application level data throughput of the protocol is the most important benefit of using this protocol over the currently used protocol. The goodput data was collected over a 365 day simulation. The results will be presented as an average amount of data per day with the time needed to leave discovery mode removed. This means that data will start being transmitted on day 138, giving 227 days that the data transfer averages are computed over. This is because the time required to leave discovery mode is a one time event at the beginning of the lifetime of a satellite, which can be completely removed by injecting the route information into the satellite by the ground station as described earlier in the discovery mode section. The simulations are performed with one data mule in each case and a 
range of one sensor satellite to four sensor satellites. The communication range assumed for every satellite in all of the results from here on out is $150 \mathrm{~km}$ and a bit-rate of $100 \mathrm{kbps}$, both chosen because it was a reasonable value to be able to find hardware to support and they are on the low end of the feasibility study.

\subsubsection{Maximum data transfer with one satellite as the origin of all the data}

In this experiment data was fed to one satellite in quantities such that it was more than the network of satellites could handle during the simulation. This was to find the maximum amount of data that could be transfered through the network if all data was coming from a single source, but could use the other satellites to get it to the data mule. The purpose for learning this information was to know if there was a single satellite that sent the majority of the information, how much would that one satellite be able to send during the simulation time. In this experiment no other satellite sent any of its own data, they only send out link state information and forwarded packets originating at the source, CP4 or MAST, but it is similar to what would happen if most satellites had very little information to send to the data mule and one satellite had large amounts. A secondary purpose of this experiment was to show that depending on the satellite used as the source the maximum amount of data that is transfered can be very different.

The $\mathrm{X}$-axis in figure 11 is the number of satellites not including the data mule that is used in the experiment, therefore one means only the source is being used and four means the source satellite and three additional satellites are included in the network. Zero is reserved for the maximum amount of data that the current implementation can handle. This connection only has a $1.2 \mathrm{kbps}$ link and 40 minutes per day maximum that the ground station is in range of a satellite. The values given in the graph for this method are ideal values calculated based on the bits per second and the 40 minutes per day. Therefore this is based on a theoretical limit using $100 \%$ of the available bandwidth with no overhead or lost packets, which is impossible to achieve. The Y-axis is the average MB per day that are 


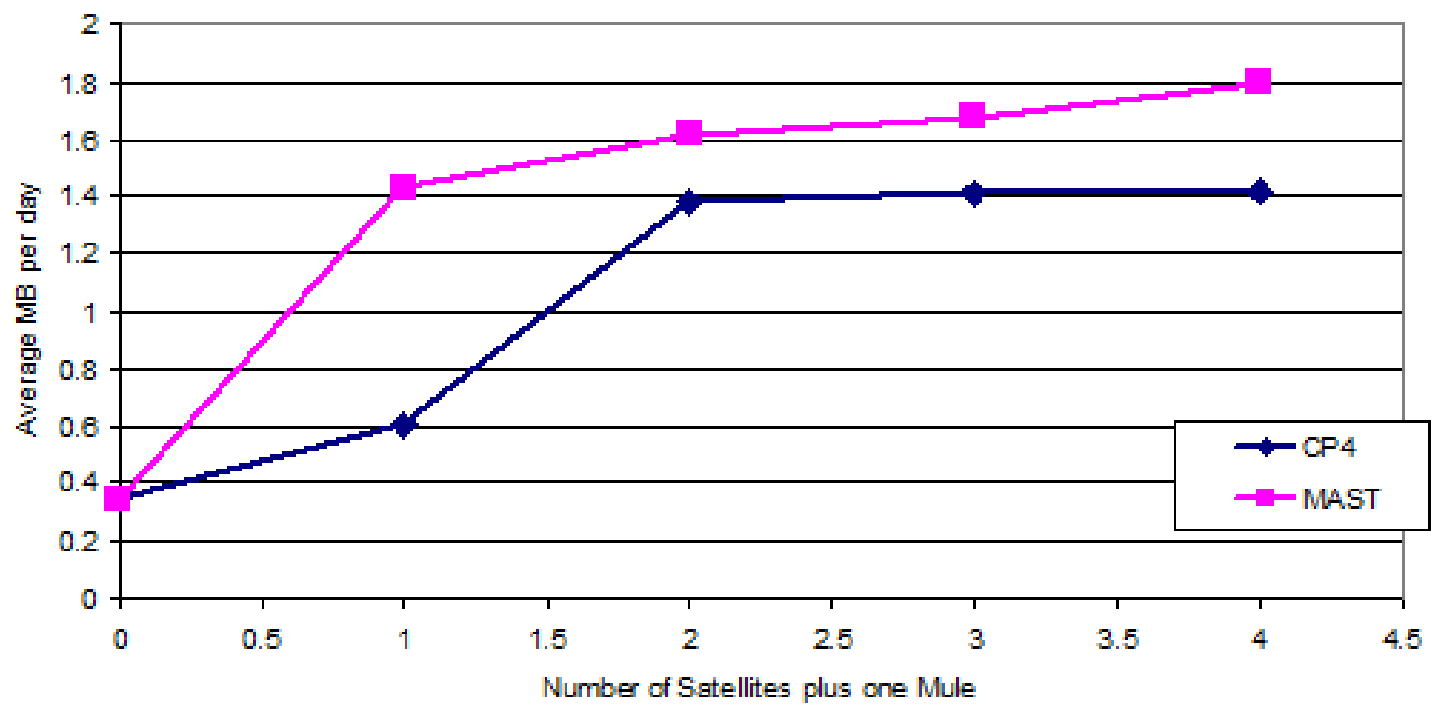

Figure 11: Maximum amount of data through network with only one satellite as the origin of the data.

transmitted from the source node to $\mathrm{CP} 3$, the data mule.

This experiment was done with two separate satellites as the source node. As stated before this is to show the difference between data transfers with different satellites. CP4 and MAST were the two chosen for this experiment because they have very different durations of connections and time between connections. CP4 has shorter connection times and time between connections. The time between connections are approximately one month and the time of the connections are approximately 3000 seconds. MAST has both longer connection times and time between connections. MAST's time between connections is approximately two and a half months, but the duration of those connections are approximately 16000 seconds.

As figure 11 shows the current implementation only achieves $0.34 \mathrm{MB}$ per day of data transfer. Both $\mathrm{CP} 4$ and MAST alone communicating to a data mule achieve much more than that. $\mathrm{CP} 4$ is approximately double with $0.6 \mathrm{MB}$ per day and MAST is almost five times better with 1.43 MB per day. With each additional satellite the amount of data per day that can be transmitted increase especially when adding the first additional satellite in 


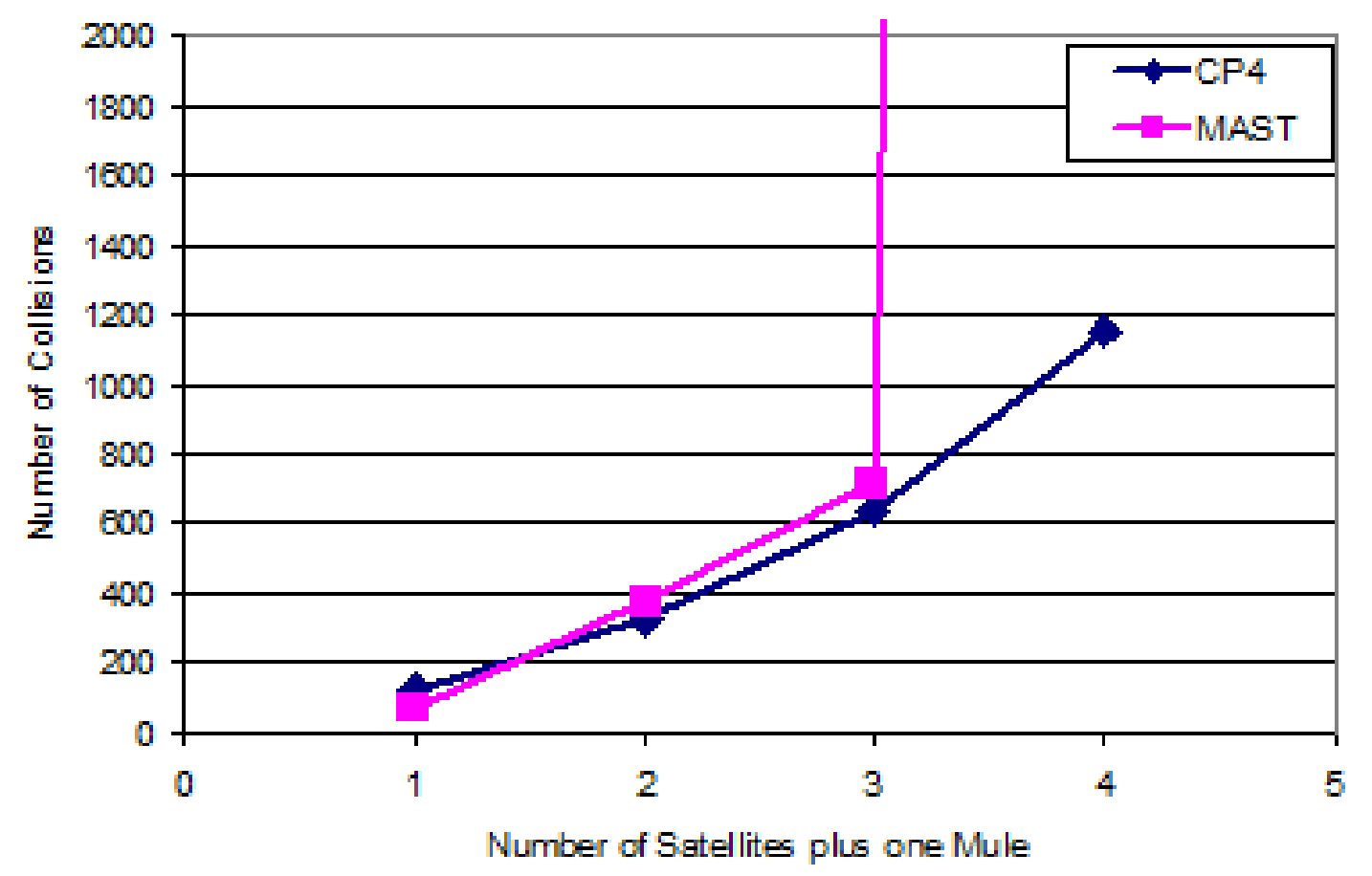

Figure 12: Collisions while sending the maximum amount of data through network from a single source.

the case of $\mathrm{CP} 4$. $\mathrm{CP} 4$ has a much lower connection time with the data mule, which limits its data transfers, but once a second satellite is added to the network which it can unload part of that data, its data rate per day more than doubles. Past this first additional satellite there are some increases in the amount of data able to be transfered but nothing nearly as significant.

Another piece of data was collected during this experiment, the number of collisions. This data shows how many packets were sent but unable to be delivered due to a collision. A large number of collisions means that there was a large amount of wasted bandwidth that could be reclaimed if a better mac protocol was implemented. Figure 12 shows that with one, two, or three satellites in the network the number of collisions is very low, less than 800 when several hundred thousand or even millions of packets are being sent. However once the 4th satellite is added to the network the collisions increases dramatically up to over 31000 in the case of MAST. 
There are two reasons for this. First of all with 4 satellites in the network transferring large amounts of data it is possible for a forwarding satellite to send the data to a different next hop then the source satellite had intended. This is because each satellite stores their own flow information, which is not shared among satellites. Even if this data was shared it would be difficult to keep all of them up to date enough to solve this problem completely due to the rarity of the exchange of this information. This can be corrected by implementing source routing, which would add more overhead to the packets, but the receiving satellite would always know where the source node had intended the data to go. The second addition that could reduce the number of collisions would be to implement a more intelligent mac protocol, most likely one that is synchronized so the satellites are always able to transmit data back and forth with high efficiency.

\subsubsection{Maximum data transfer when limited to a single hop}

In this experiment each satellite always had data to send through the network, but could only send data directly to the data mule. This simulates how the network would act if there was no inter-satellite communications except for the data mule. The simulations was run using a single satellite then increased to multiple satellites all sending data to the data mule.

Figure 13 shows the number of MB per day that are transmitted on average depending on the number of satellites in the network. Zero is once again reserved for the current implementation, with an average of $0.34 \mathrm{MB}$ per day. One satellite is computed through the average values provided from two simulations, one using CP4 and the other using MAST. This was done because MAST had a very large value and CP4 had a very small value and average of the two was more appropriate. On average with one satellite in the network approximately $1 \mathrm{MB}$ is sent per day. Which is then doubled when the number of satellites is increased to two. This increase makes sense because with one satellite it was an average of CP4 and MAST but with two satellites it was the addition of these two satellites values.

With the addition of the next two satellites less improvement was found. However, this 


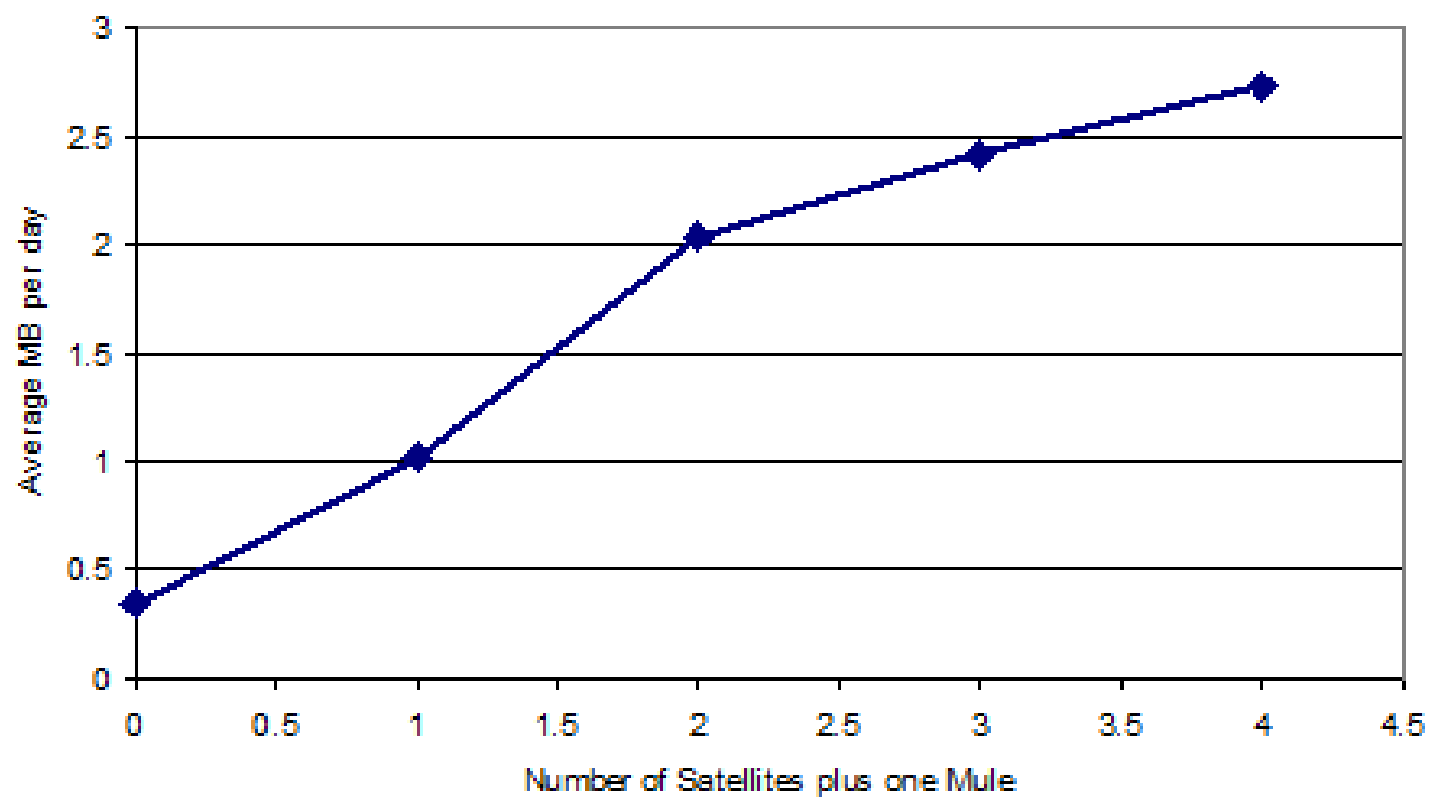

Figure 13: Maximum MBs transfered after a 365day simulation when sending data from every satellite to CP3 limited to a single hop

isn't due to a problem with the protocol, but rather the orbits of SAUDI4 and SAUDI6 do not have as long of durations as CP4 and MAST do which means their improvement isn't as pronounced. The data rate when having the satellites communicate with only the data mule is directly related to the duration of their own connection to the data mule and not on the addition of more satellites in the network. This means that the satellites generally do not come into contact with the data mule at the same time, there is at least some time between connections of different satellites and the data mule. This could however become a problem once the connection between the data mule and the ground station is dealt with. If the data mule has to transmit too much data that its own link to the ground station cannot support it, the data mule may become a bottleneck. This however is out of the scope of this project and will be left for future research.

The number of collisions during this simulation was directly related to the number of satellites in the network. The number of collisions was mainly due to the transfer of link state information between the satellites and is very low in comparison to the millions of 


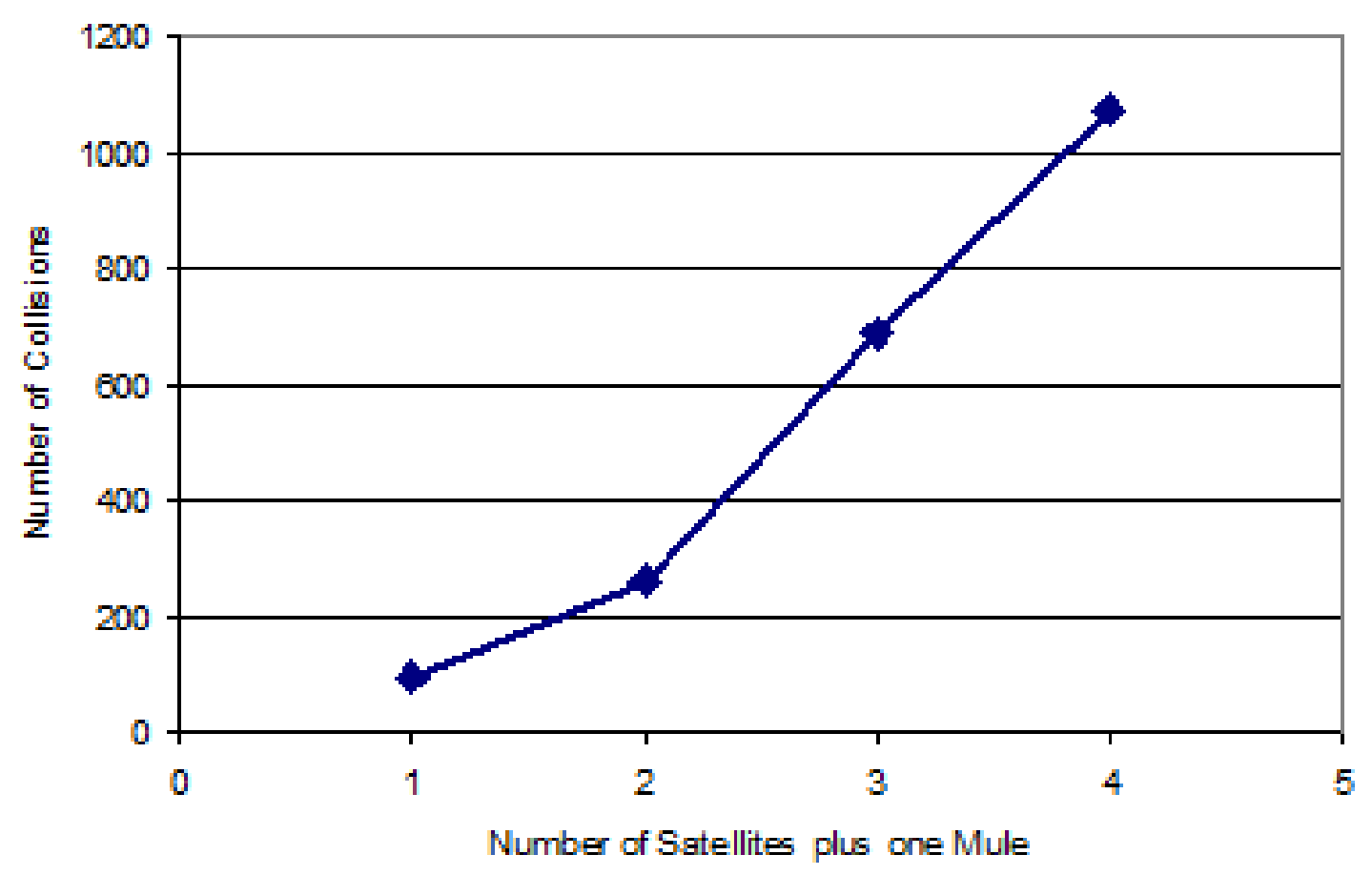

Figure 14: Collisions after a 365day simulation when sending maximum data from every satellite to CP3 limited to a single hop

packets sent. This is partially due to the semi-synchronized transfer of data once large amounts of data are sent.

The final piece of information gathered was the distribution of the information that was transfered to the data mule. This is in support of the previously mentioned hypothesis that the amount of data sent to the data mule was proportional to the amount of time that the two satellites are in range. Another purpose of this graph is to compare the single hop version to the multi hop version in the next section, therefore more will be said about this graph in the following section.

\subsubsection{Maximum data transfer with multiple hops}

This experiment is much like the previous one, but the satellites could send data through any route they could find. The satellites still have an unlimited amount of data to send. This 


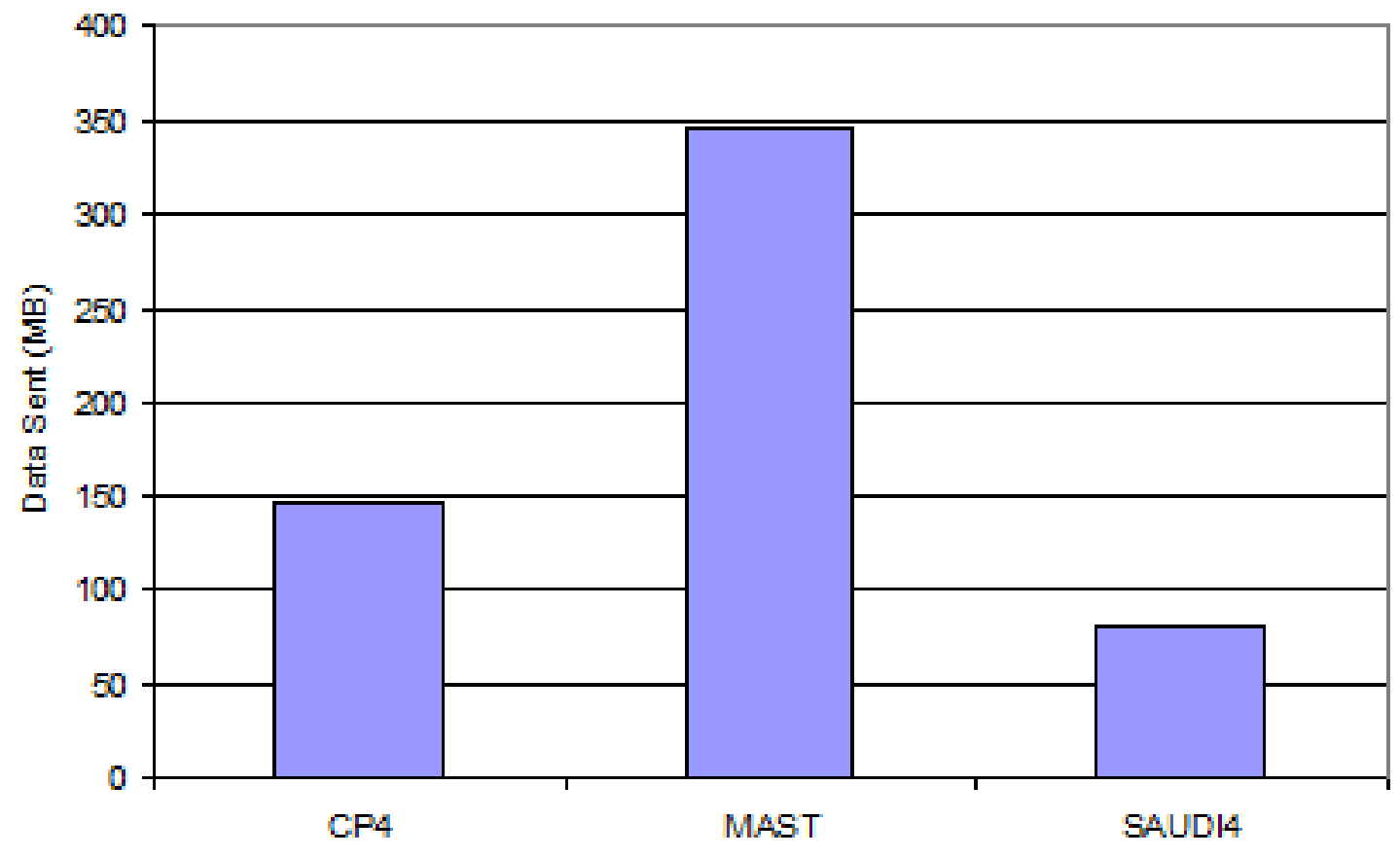

Figure 15: Distribution of data Sent after a 365day simulation when sending data from every satellite to CP3 limited to a single hop

experiment compares the single hop to the multi-hop by the total amount of goodput they are able to provide. This experiment is interesting because it is hypothesized that when all satellites are sending the maximum amount of data, using multiple hops will not provide any improvement.

Figure 16 shows the results of this simulation. This simulation was only performed until a maximum of 3 satellites in the network because when the fourth was included there were so many packet collisions that no usable data was recovered about the addition of the final satellite. However with the first three satellite there was approximately the same amount of data that was transfered between the satellites. There was a slight decrease in the value once three satellites were used, but not a significant one. This is exactly as expected because if every satellite is fully utilizing its link to the ground station then there is no additional room for any other satellite to transmit its data through that satellite.

The number of collisions for this simulation was incredible as shown in figure 17. Due 


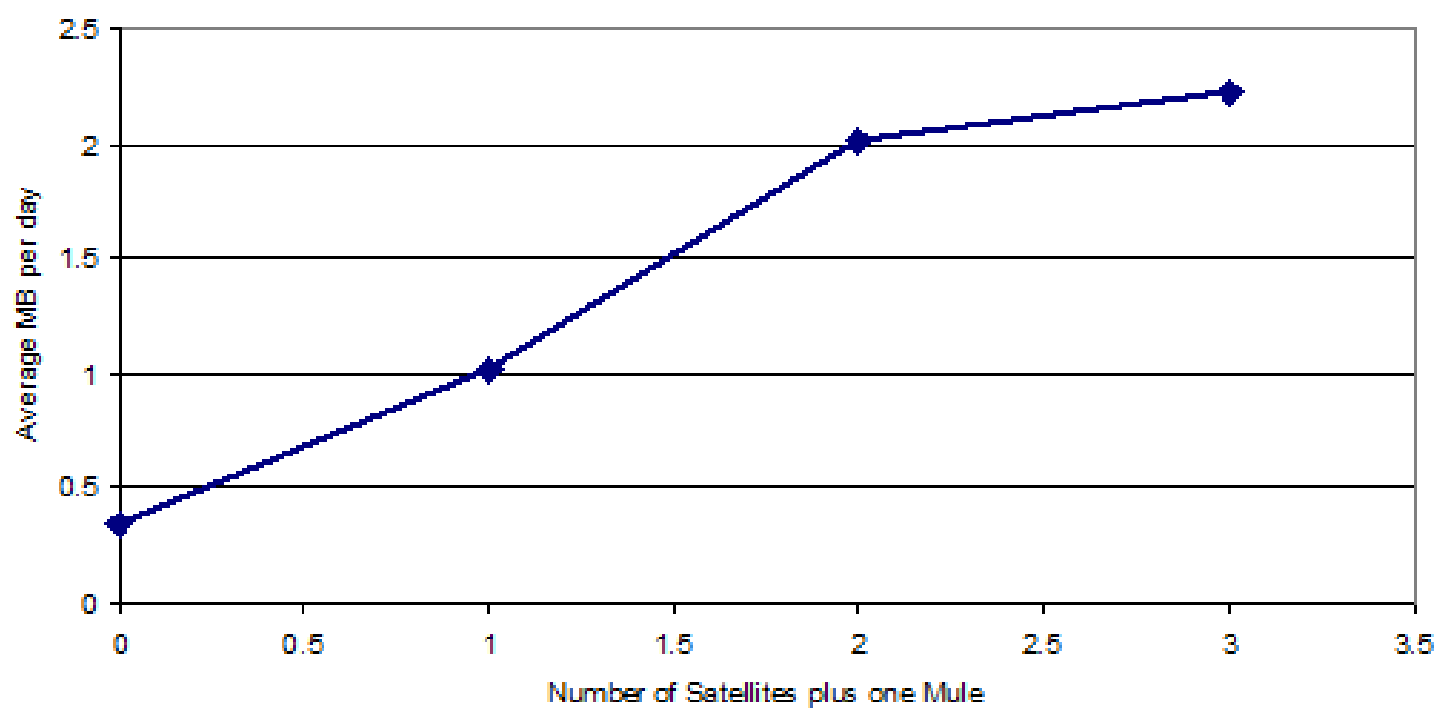

Figure 16: Maximum MBs transfered after a 365day simulation when sending data from every satellite to CP3 allowing multiple hops

to every satellite sending as much data as possible through every possible link, there was a large number of collisions. As can be seen the number of collisions with three satellites is over 40000, this is the reason why the fourth satellite was omitted from this simulation, the number of collisions was even higher and it interfered with the function of the network. These problems can once again be mitigated by source routing and a more sophisticated mac protocol.

Notice the amount of data transfered between the satellites is very similar, but there is a much larger number of collisions. This is possible because the satellites purposefully don't fill up the full duration of their connection to leave room for important data going through and to have a little extra room just in case a connection is cut short for some reason an data needs to be sent out on the next connection.

Once again in this simulation the distribution of the data sent through the network was gathered. Comparing the graphs in figures 15 and 18 you can see that the distributions are almost identical. This shows that when the network is maxed out then it is exactly the same as all satellites communicating with the data mule. Therefore the multi-hop aspect doesn't 


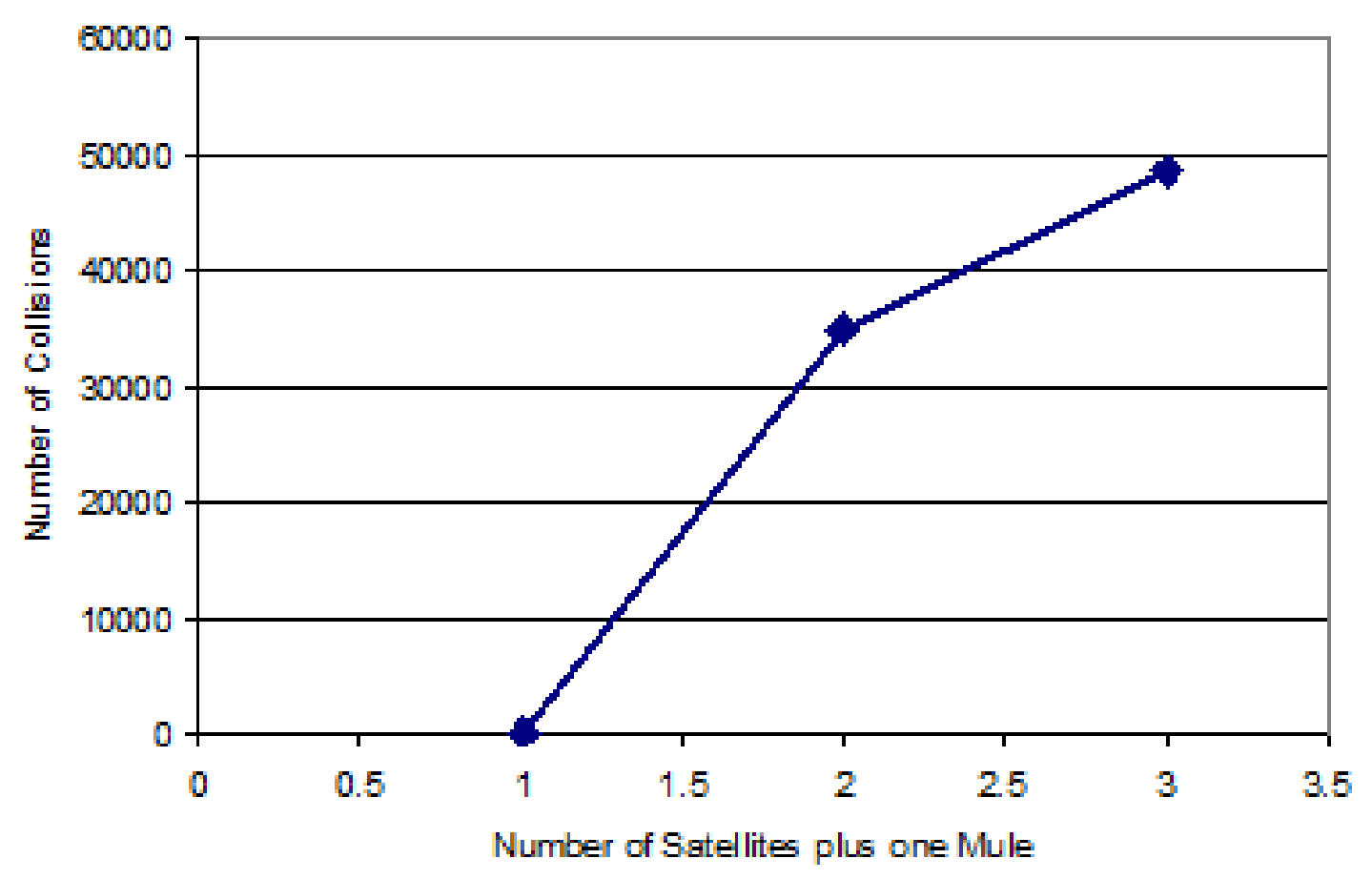

Figure 17: Collisions after a 365day simulation when sending maximum data from every satellite to CP3 allowing multiple hops

help, but also doesn't hurt the network.

\subsection{End-to-end Delay}

End-to-end delay of large files is another benefit to using this protocol. The results below show that using this protocol even with a naive mac protocol in use, there is still significant improvement to end to end delays for larger files. The results also show that the delay times achieved as well as the amount of data that can be sent in those times differs greatly between different satellites due to the differences in orbits.

\subsubsection{Sending 125MB file from CP4 to CP3 (data mule)}

In the first simulation a $125 \mathrm{MB}$ file was sent from $\mathrm{CP} 4$ to $\mathrm{CP} 3$. The simulation was run using different numbers of satellites in the system starting with only the two and ending 


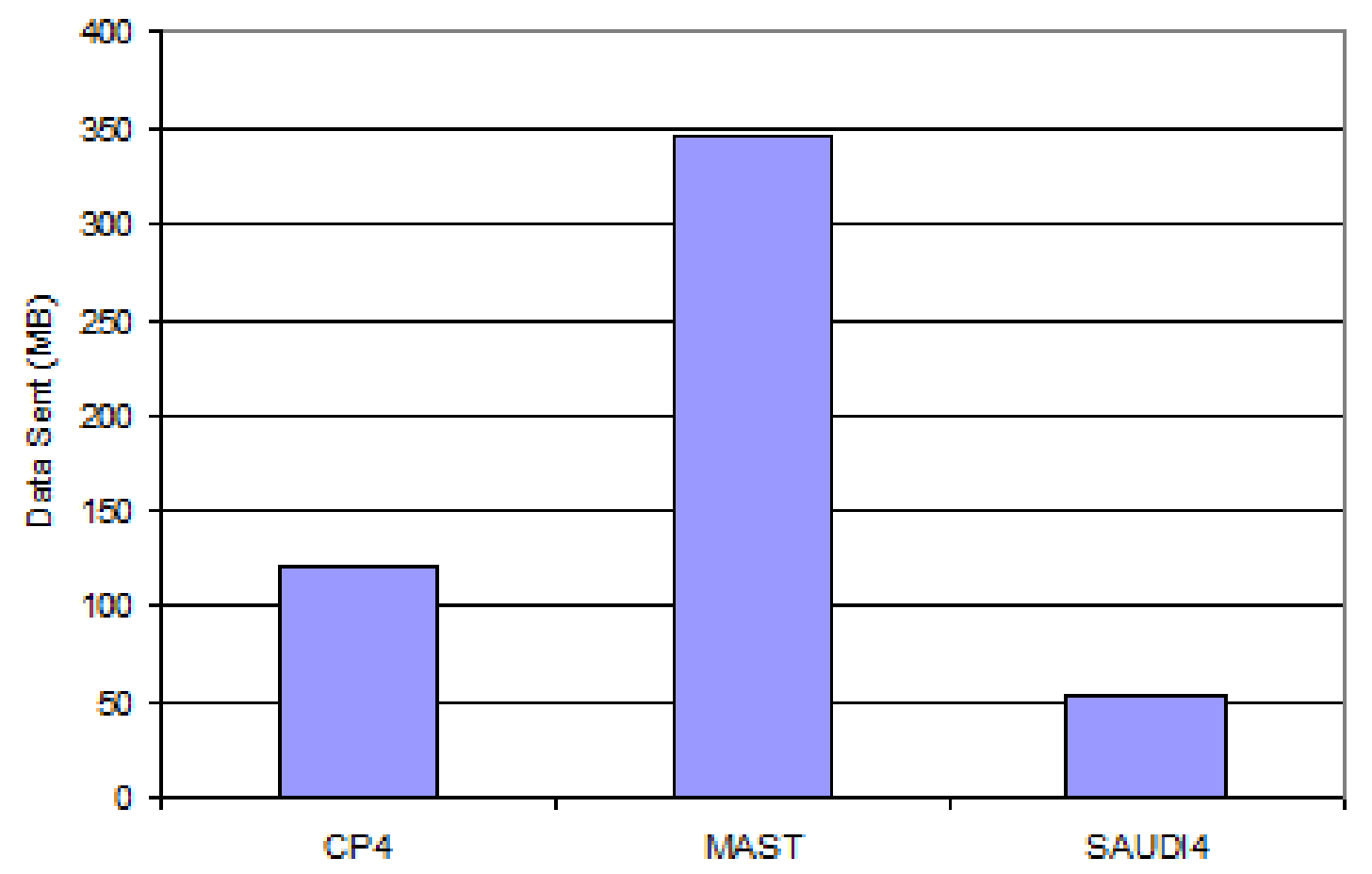

Figure 18: Distribution of sent files after a 365day simulation when sending maximum data from every satellite to CP3 allowing multiple hops.

at three additional satellites (five total). When adding the extra satellites there is no data originating from them. These additional satellites will forward the data for CP4 but do not have any of their own data to send. The purpose of this experiment is to see the effect of having only a single satellite in comparison to multiple satellites when one of those satellites has a large burst of information it needs to send. This could happen in the real world if a satellite was collecting and analyzing large amounts of data and compiling it all into one large file before sending it back to Earth.

In figure 19 the $\mathrm{X}$-axis is the number of satellites in the network, not including the Data Mule. The Y-axis is the number of days that it took CP3 to receive the last byte of the $125 \mathrm{MB}$ file. The number of satellites at zero signifies the current method of sending data between a satellite and Earth directly, which only has a 1.2kbps link and 40 minutes per day maximum that the ground station is in range of the satellite. The values given in the 


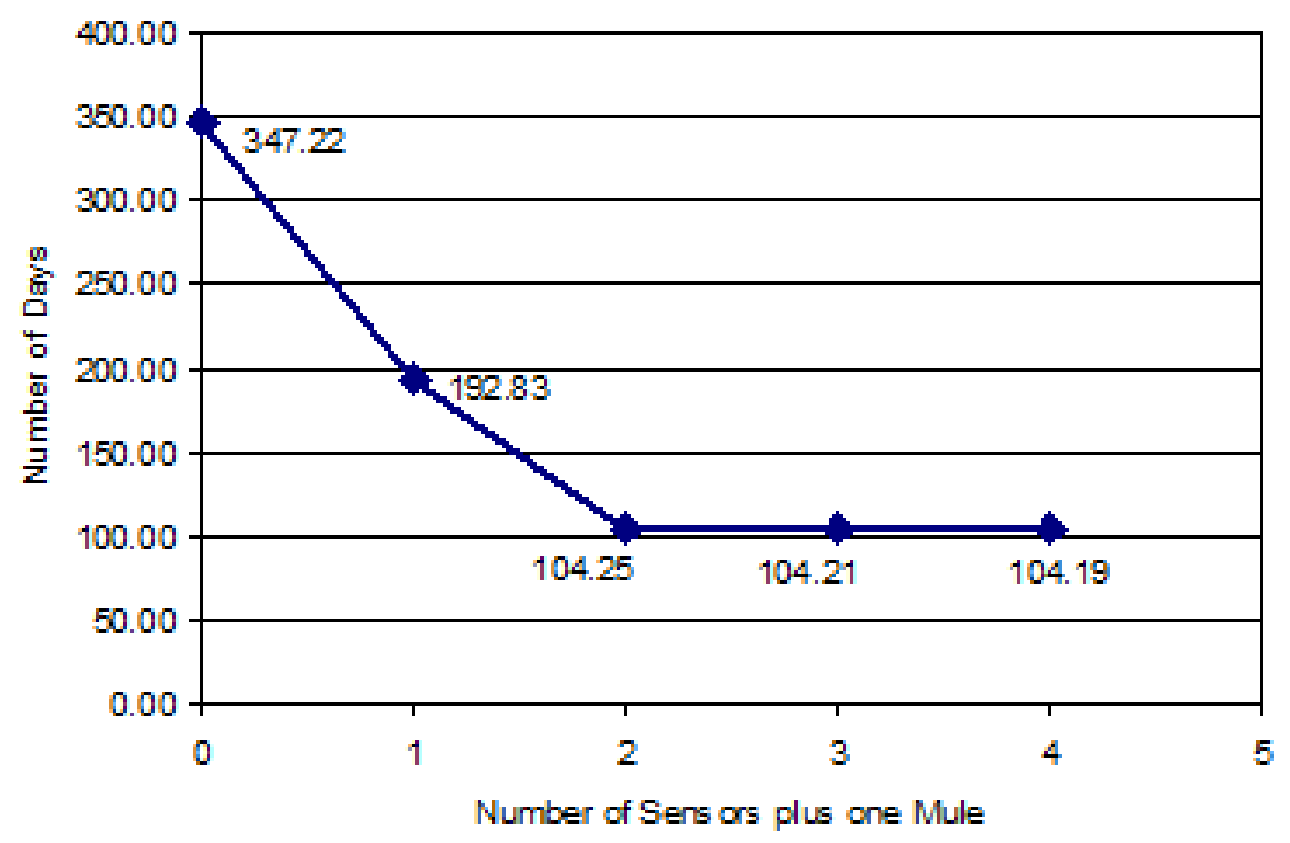

Figure 19: End-to-end Delay of 125MB file from CP4 to CP3. (CP4 is the only satellite sending data)

graph for this method are ideal values calculated based on the bits per second and the 40 minutes per day. Therefore this is based on a theoretical limit using $100 \%$ of the available bandwidth with no overhead or lost packets, which is impossible to achieve.

The time it would take even using this theoretical maximum to send $125 \mathrm{MB}$ of data would be 347.22 days or almost a full year. Running a simulation using a data mule which will have a much larger connection down to the ground station, CP4 is able to transmit the full $125 \mathrm{MB}$ of data to CP4 (the data mule) in only 192.83 days. When adding additional satellites to the network this number decreases even more down to 104 days, less than one third of what it would take the current implementation.

Figure 20 shows the number of collisions that occurred during this experiment. Because there was only one satellite sending data in one direction the collisions are very low when compared to the number of sent and received packets shown in figure 21 . The collisions are due to the connecting and transferring of information between the satellites usually before 


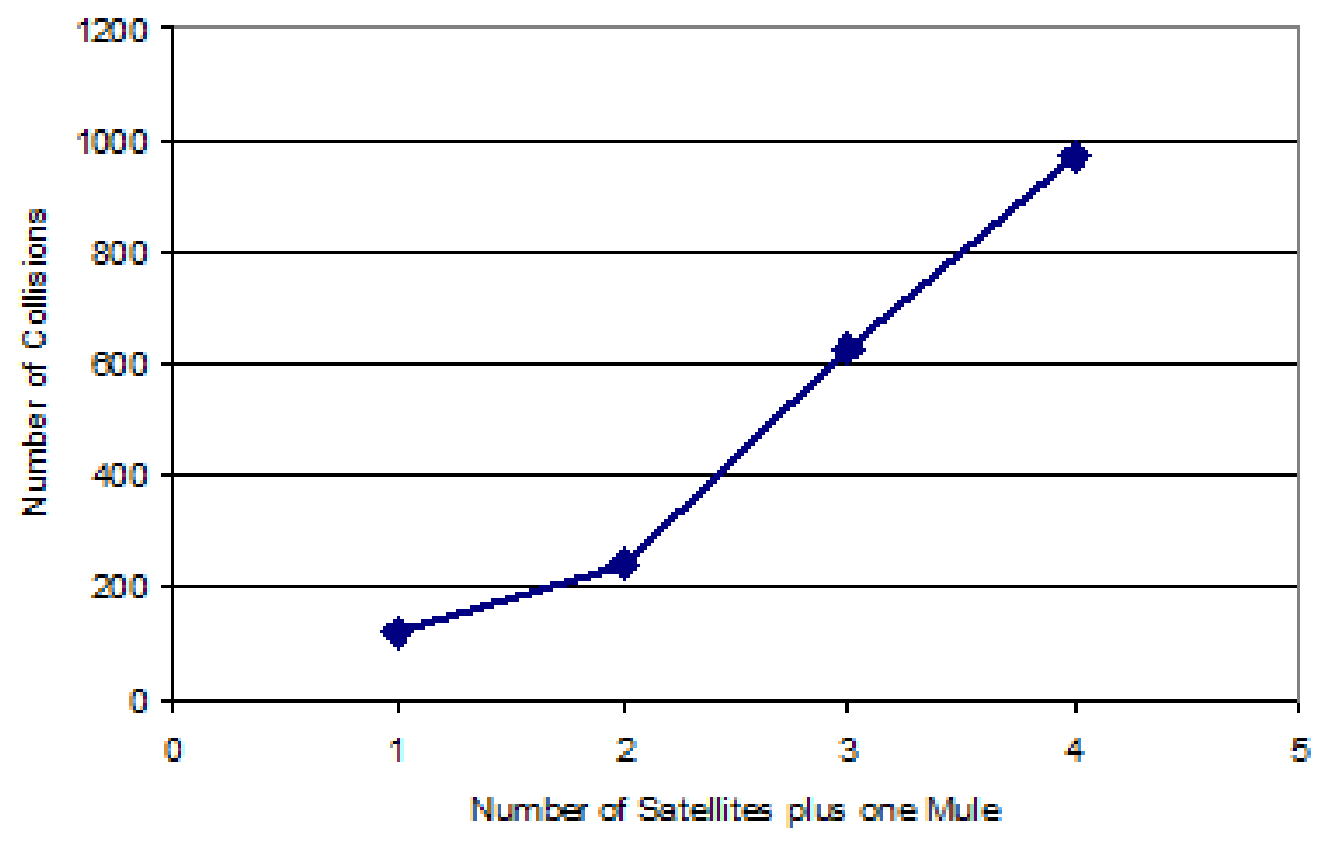

Figure 20: Collisions while sending a 125MB file from CP4 to CP3. (CP4 is the only satellite sending data)

the actual transfer of data has begun. Once the data is transfered the satellites are almost completely synchronized so the acknowledgments are only sent when the receiving satellite knows the sending satellite has stopped sending data.

The number of packets sent does increase at a linear rate when adding new satellites to the system, which is to be expected because more packets are needed to be sent to keep up the link state information and more packets are able to be sent between satellites to get the data to the data mule faster.

\subsubsection{Sending 250MB from MAST to CP3 (data mule)}

The next experiment performed was sending a larger file, 250MB, from MAST to CP3. A larger file was used in this experiment because MAST tends to have longer passes than $\mathrm{CP} 4$ does and can send more information during those passes. This experiment shows that depending on the orbit of the satellite there can be large differences in the amount of data 


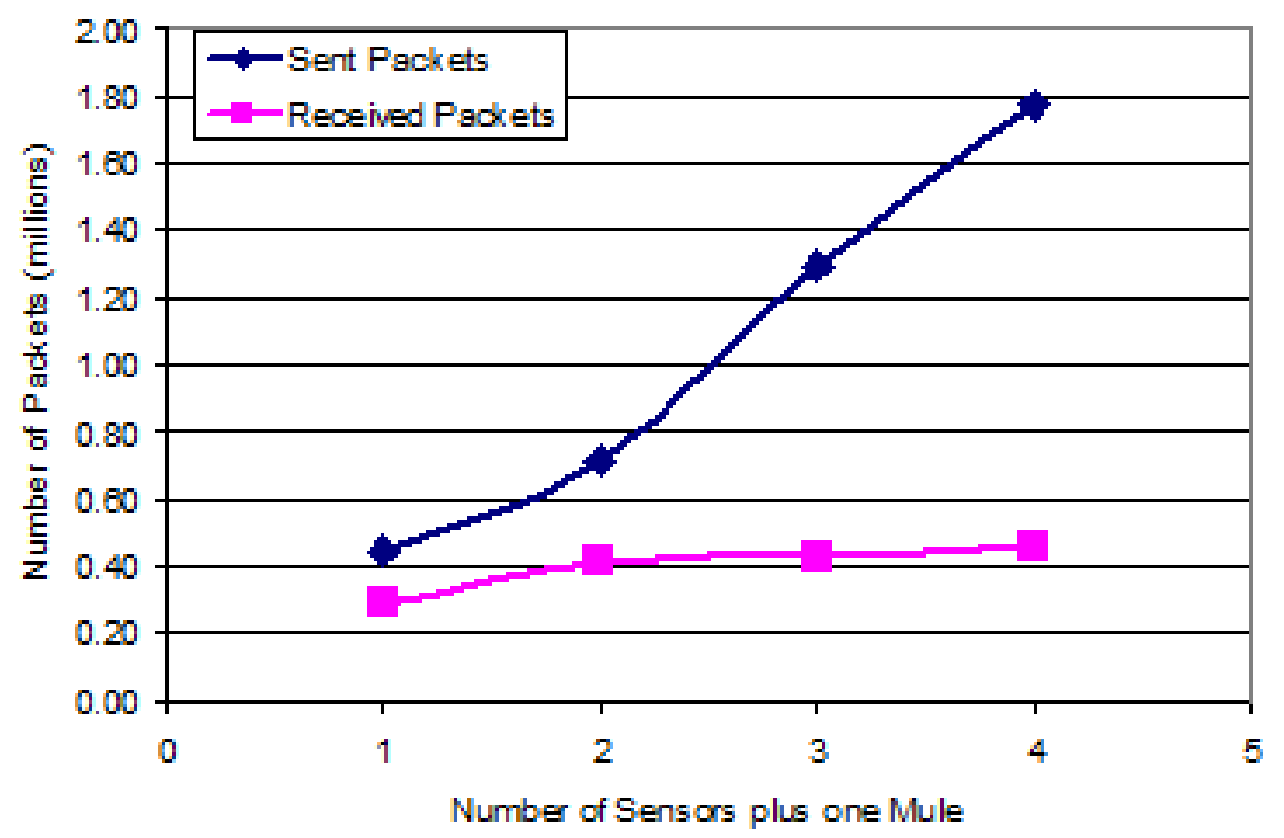

Figure 21: Number of sent and received packets while sending a $125 \mathrm{MB}$ file from $\mathrm{CP} 4$ to $\mathrm{CP} 3$. (CP4 is the only satellite sending data)

and the length of time it takes that data to get from a source to the data mule.

Once again the $\mathrm{X}$-axis in figure 22 shows the number of satellites in the network not including the data mule, none of which are sending any data except for MAST. The Yaxis is the number of days it takes the last byte of the $250 \mathrm{MB}$ file to get to $\mathrm{CP} 3$, the data mule and the zero value on the $\mathrm{X}$-axis shows the theoretical minimum time it would take to send a 250MB file. Once again this value can never be accomplished because it assumes a perfectly efficient transfer of data with no overhead, no loss of packets, using $100 \%$ of the bandwidth.

The currently used implementation would take more than 694.44 days to complete the transfer of a $250 \mathrm{MB}$ file. The transfer of the same amount of information plus sending overhead with one satellite transferring data to a data mule, only takes 178.57 days. Notice that in the above example only $125 \mathrm{MB}$ was sent, half of what is sent in this experiment, but this experiment does not take twice the time to transmit that data. This is because the 


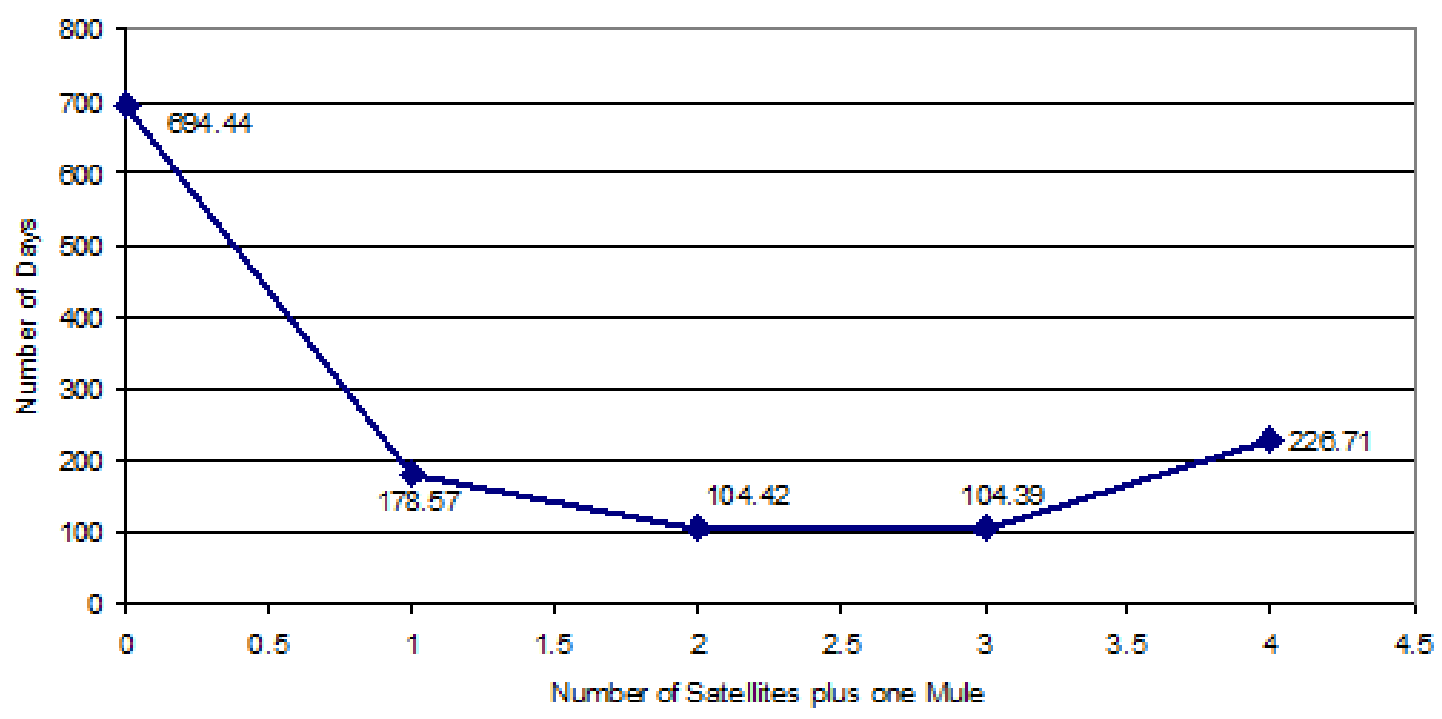

Figure 22: End-to-end Delay of 250MB file from MAST to CP3. (MAST is the only satellite sending data)

orbits of CP4 and MAST are different giving MAST the ability to send larger amounts of information to $\mathrm{CP} 3$, the data mule, faster. Once additional satellites are added to the network the amount of time it takes for the file to arrive drops down to 104 days the same as it did in the previous example. This is once again due to the orbit of MAST being better suited to transmit large amounts of data from itself to the data mule.

One thing to note however is that the amount of time to transfer the file actually goes up when three additional satellites are used in the network. This is caused by a large number of collisions occurring during the transfer of data. As you can see in figure 23 the number of collisions does increase with the addition of each new satellite into the network, but once the third extra satellite is added the number of collisions goes from about 700 to over 5000 . Because of the large amount of data that is being transfered and the lack of knowledge of where each satellite expects the data to go information is being sent both directions at once causing numerous collisions and limiting the bandwidth of the system. This can be mitigated with the change to source routing. If each satellite is told where each packet it receives is supposed to go then there will not be any mix-up of sending data to an already 


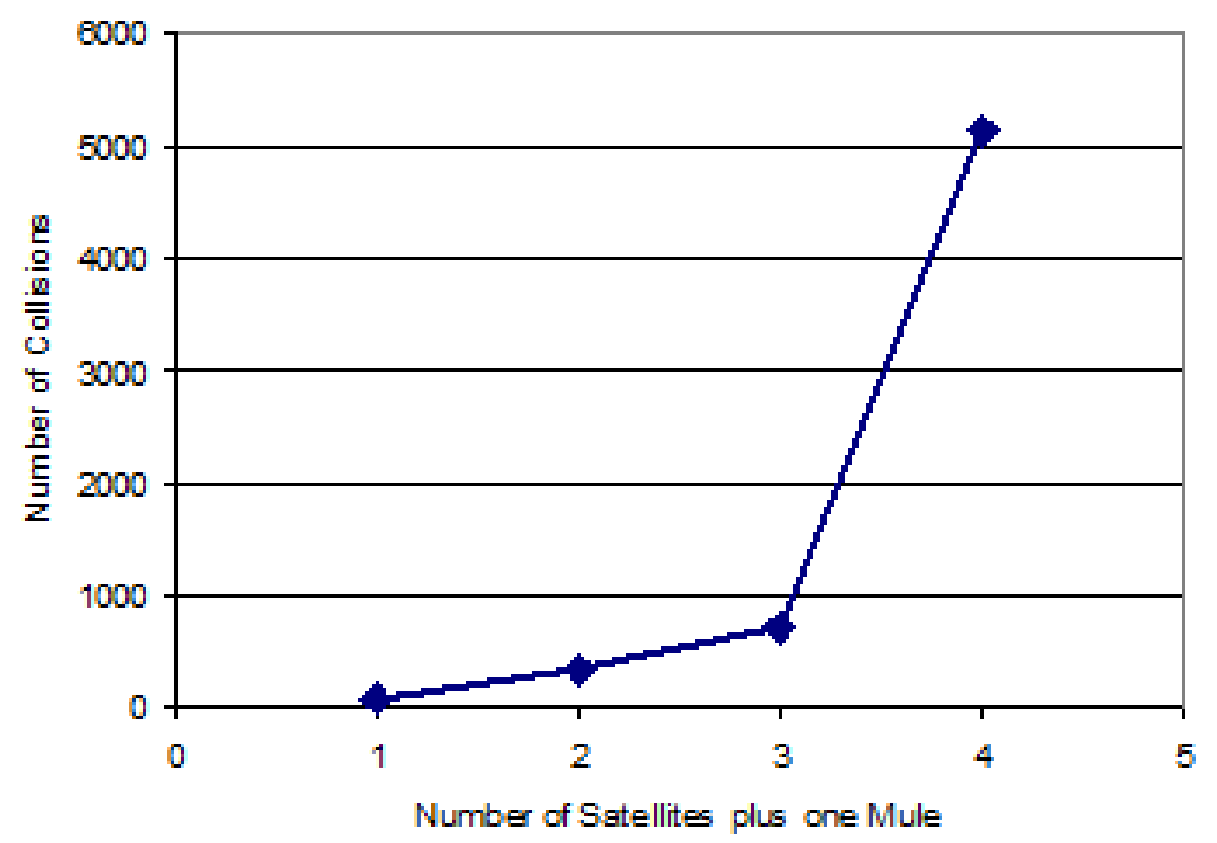

Figure 23: Collisions while sending a 250MB file from MAST to CP3. (MAST is the only satellite sending data)

over burdened link.

On a final note on this experiment, the number of sent and recieved packets was also tracked like in the previous experiment. The number of packets sent was higher as to be expected due to the larger amount of data being sent between nodes, but both figure 21 and 24 show a very similar almost linear growth in the number of packets sent and received.

\subsubsection{Sending $1 \mathrm{MB}$ per day per satellite}

This experiment starts sending one megabyte per day per satellite starting at the moment that the satellites have the required information to leave discovery mode. The purpose of this experiment is to see how the protocol will react to a steady stream of smaller files. The current protocol is only able to send approximately a $343 \mathrm{kB}$ file every day, making it take three days to send a one megabyte file. Which means that early on the end-to-end delay will be close to three days, but as the number of days increases that the $1 \mathrm{MB}$ per day is 


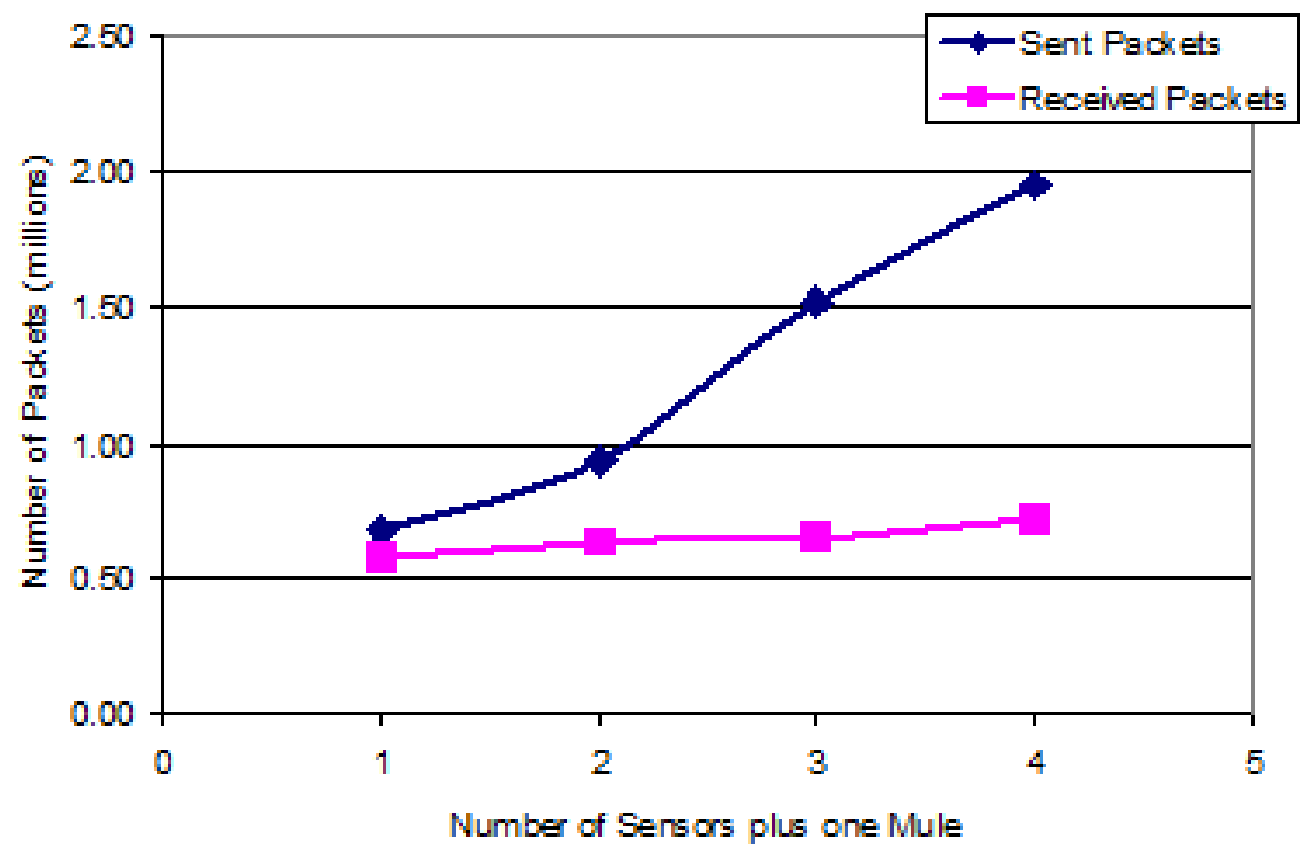

Figure 24: Number of sent and received packets while sending a 250MB file from MAST to $\mathrm{CP}$. (MAST is the only satellite sending data)

sent the longer the end-to-end delay gets. The end-to-end for the current protocol can be estimated as $2 N+1$ because every day that passes only one third of a file can be processed and three more days of data transfer is added to the system. Table 6.2.3 shows this pattern. On day one the file will take three days to complete, day two the file will have to wait two more days for the first file to complete then three days for itself, the third file will then have to wait one day for the first file, three days for the second, then three more for itself. As you can see a pattern arises in the form of the previously mentioned $2 N+1$.

If you remember from high school math when taking the average of a pattern such as counting all odd number (which is what $2 N+1$ breaks down to) all you need to do is add the first number, 3, to the final number at day 227 (this is the number of days in which $1 \mathrm{MB}$ is send every day), $2 * 227+1=445$. This value comes out to $(3+445) / 2=229$. However the simulation only takes into account the end-to-end delay times of files which have already finished transferring so the same must be done for this estimate, which would be the average 


\begin{tabular}{|c|c|c|c|}
\hline Day 1 & Day 2 & Day 3 & Day N \\
\hline 3 & 5 & 7 & $2 N+1$ \\
\hline
\end{tabular}

Table 2: Table depicting the pattern of sending a $1 \mathrm{MB}$ file per day directly to the ground station using a $1.2 \mathrm{kbps}$ link and assuming $40 \mathrm{~min} /$ day contact

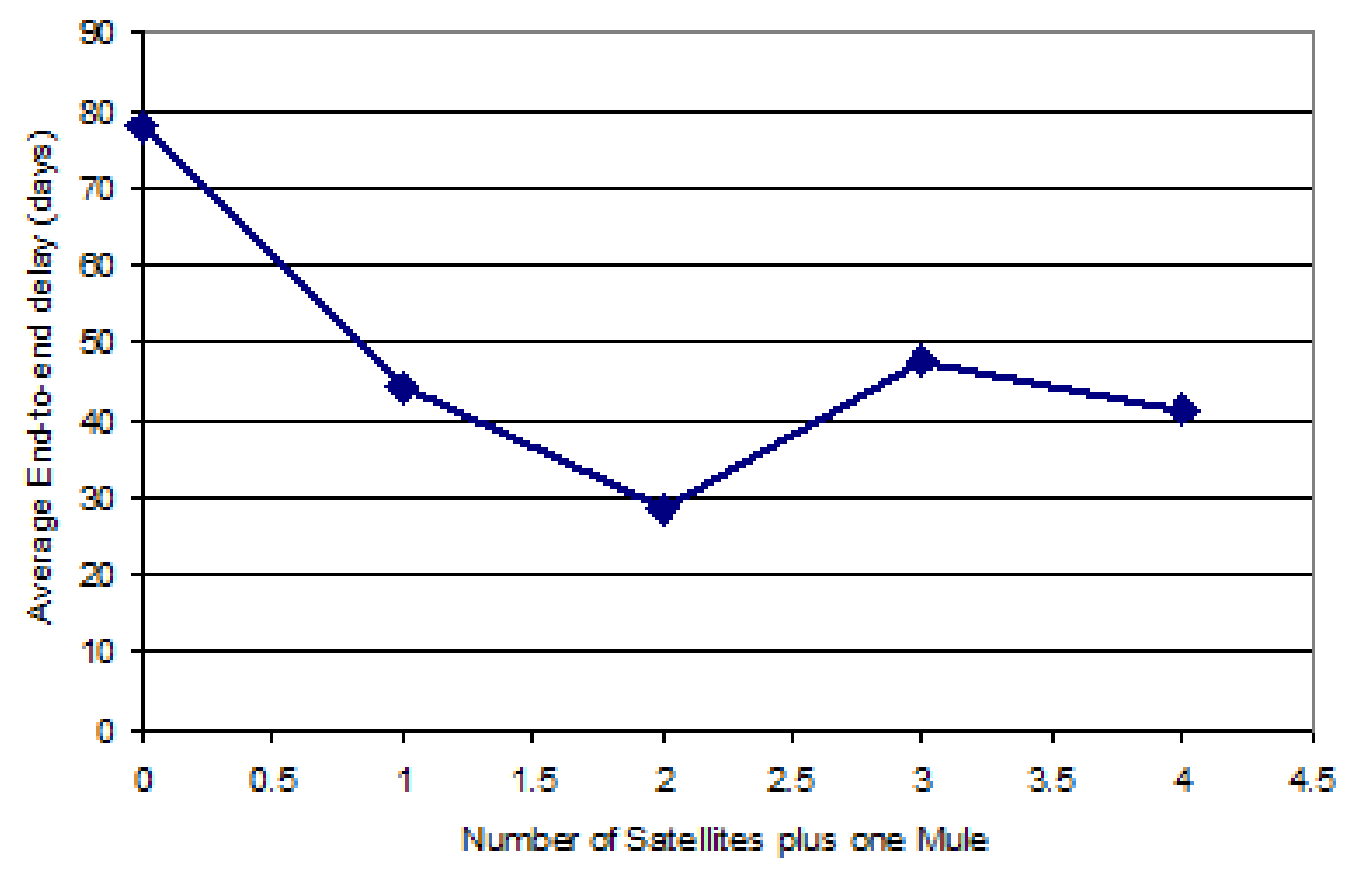

Figure 25: Average Delays Sending 1MB per day from every satellite to CP3

end-to-end delay of the number of files that are able to be sent in 227 days. Because each file takes 3 days it is possible to complete only about 76 files, the last of which was started on the 76th day. Therefore back to the equations above $(3+2 * 76+1) / 2=78$ days

Now that a basis for comparison has been established it is time to discuss the results of the protocol. With only one satellite in the network transmitting its data to the data mule the average end-to-end delay came out to be 44.42 days. This value is actually the average of two simulations done using CP4 and MAST. CP4 had a much longer average because it does not send nearly as much data MAST does, this value came out to be 56.76 days, still much below the 78 day average that the current protocol has. MAST on the other hand has large data transfer abilities even though it comes into contact with the data mule less 


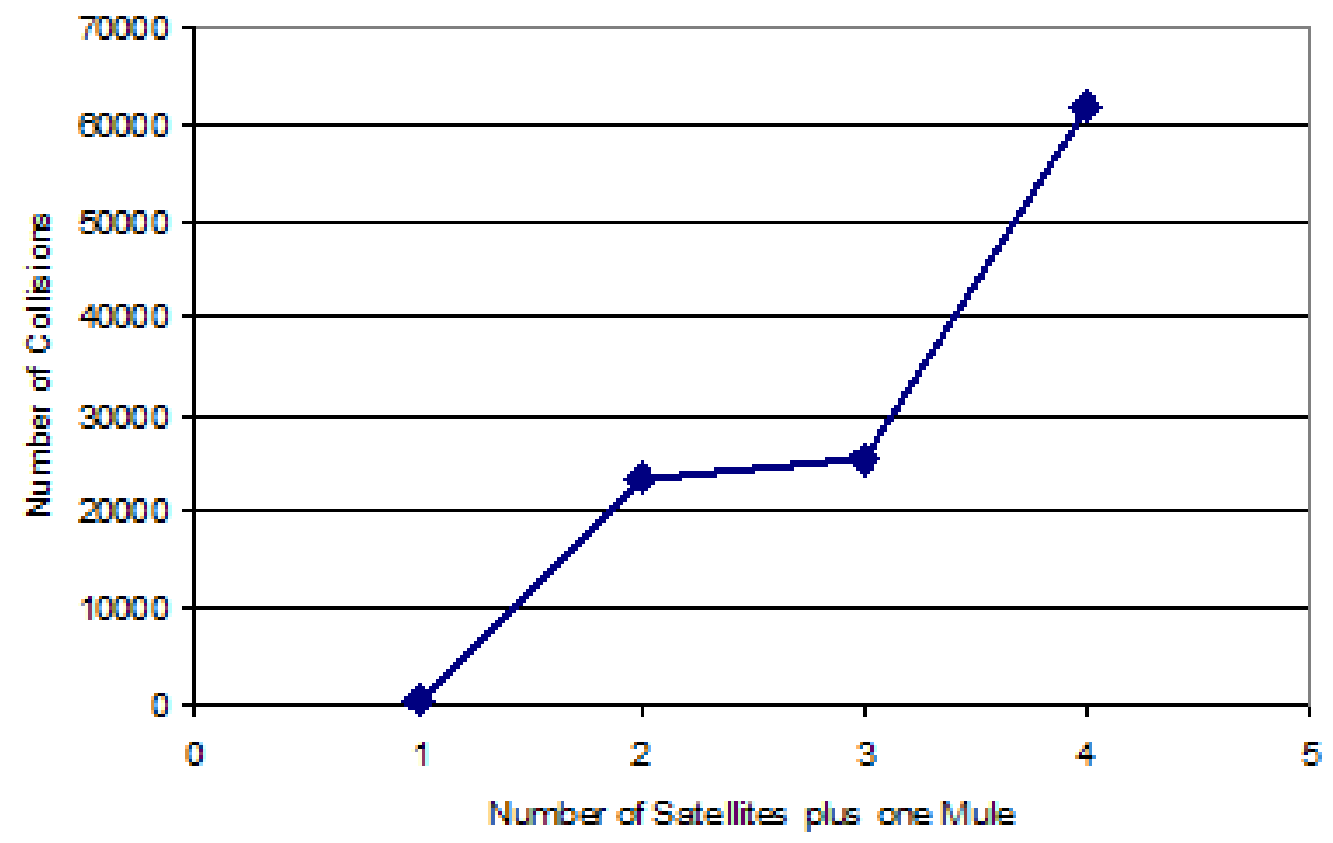

Figure 26: Collisions while sending $1 \mathrm{MB}$ per day from every satellite to $\mathrm{CP} 3$

often, with an average of 32.09 days. When adding a second satellite into the network, both CP4 and MAST, allowing them to use one another to transmit the files to the data mule, the average number of days per file drops to 28.5 .

Once more than two satellites are added to the network however the average goes back up. This is due to the two new satellites having less ability to send these files which forces them to rely on MAST and CP4 to lower the end-to-end delay time along with a large number of collisions that is caused by not knowing where the other satellites are sending information and not knowing where a packet that needs to be forwarded was desired to go by the source. Therefore by implementing source routing as well as a more intelligent mac protocol these values can be greatly decreased.

\subsection{Verification of Assumptions}

In the design of this protocol there were a few assumptions that were made including that the data mule would not act as a bottleneck and that the satellite's orbits truly are pre- 


\begin{tabular}{c|c} 
Sensor (bps) & Mule (bps) \\
$1.2 \mathrm{k}$ & $0.47 \mathrm{k}$ \\
$10 \mathrm{k}$ & $4.7 \mathrm{k}$ \\
$100 \mathrm{k}$ & $47 \mathrm{k}$ \\
$1 \mathrm{M}$ & $470 \mathrm{k}$ \\
$10 \mathrm{M}$ & $4.7 \mathrm{M}$ \\
$50 \mathrm{M}$ & $21.4 \mathrm{M}$
\end{tabular}

Table 3: MULE data rates required to not be a bottleneck, using 40 minutes per day exposure time

dictable. This section shows that these assumptions are reasonable.

\subsubsection{Data Mule Capacities}

As stated before the graphs in the previous section assume that the data mules that are being used are not acting as a bottleneck in the system. Even though this data mule needs to transmit a longer distance down to the ground station it should be able to get much higher speeds than the sensor currently are able to transmit. This is due to the larger size and less power constraints on the data mule. With that said Table 3 shows the bit rates needed by the mule compared to the sensor bit rates. Table 3 assumes two sensors in the network that always send the maximum amount of data and a data mule that has 40 minutes per day exposure time. As the table shows when there are two sensors in the network the data mule needs to send data to the ground station at less than half the speed of the sensors. As the number of sensors increases the bit rate required by the data mule will also need to increase, but because these networks are going to be sparse with very few satellites this shouldn't be a problem. However, if too many satellites are put into the network the solution is to put more data mules in space at the same time. If there are equal bit rates in both the sensors and the data mule then there should be approximately one data mule per four satellites.

\subsubsection{Predictability of Orbits}

This protocol assumes that the time between passes in the orbits are predictable. This section will show that this assumption is true. Figure27 shows the time between passes 


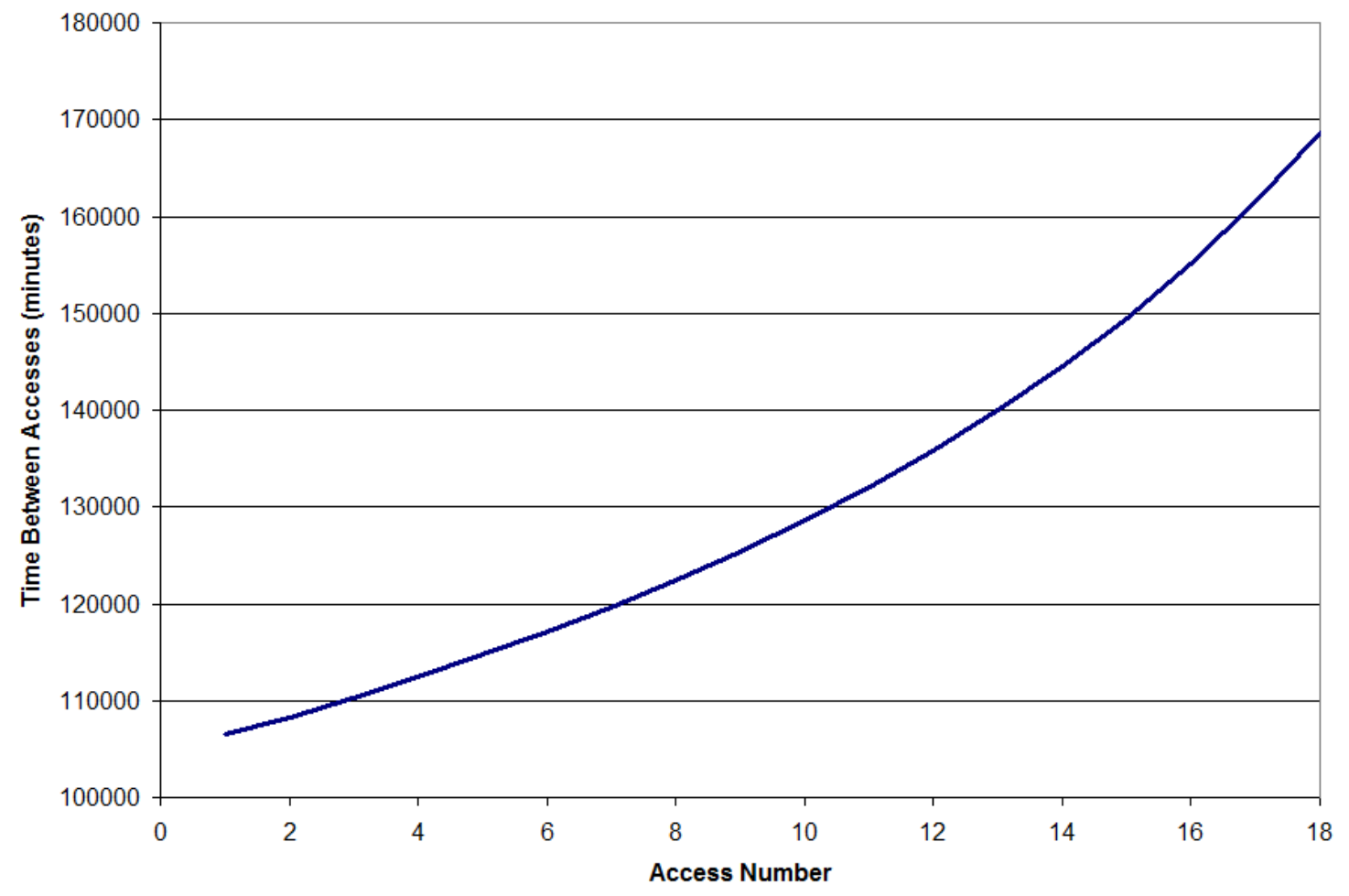

Figure 27: Time between accesses over 5 years CP3 to MAST (CubeSat Satellites)

of CP3 and MAST over the span of 5 years. There is an obvious trend to this data that is approximately exponential. The graph also shows that the time between passes is never more than 10 percent different. Figure 28 shows the time between passes of CP4 and SAUDICOMSAT6 over the span of five years also. This graph also have a very distinct trend, but is decreasing exponentially instead of increasing, but once again there is never more than a 10 percent difference between passes. This data shows that the way this is currently implemented with the 10 percent buffers is good enough to hold over long periods of time, but isn't the most accurate estimating. A more accurate estimation would be to track the changes in the time between and fit it to a linear equation especially between single accesses. 


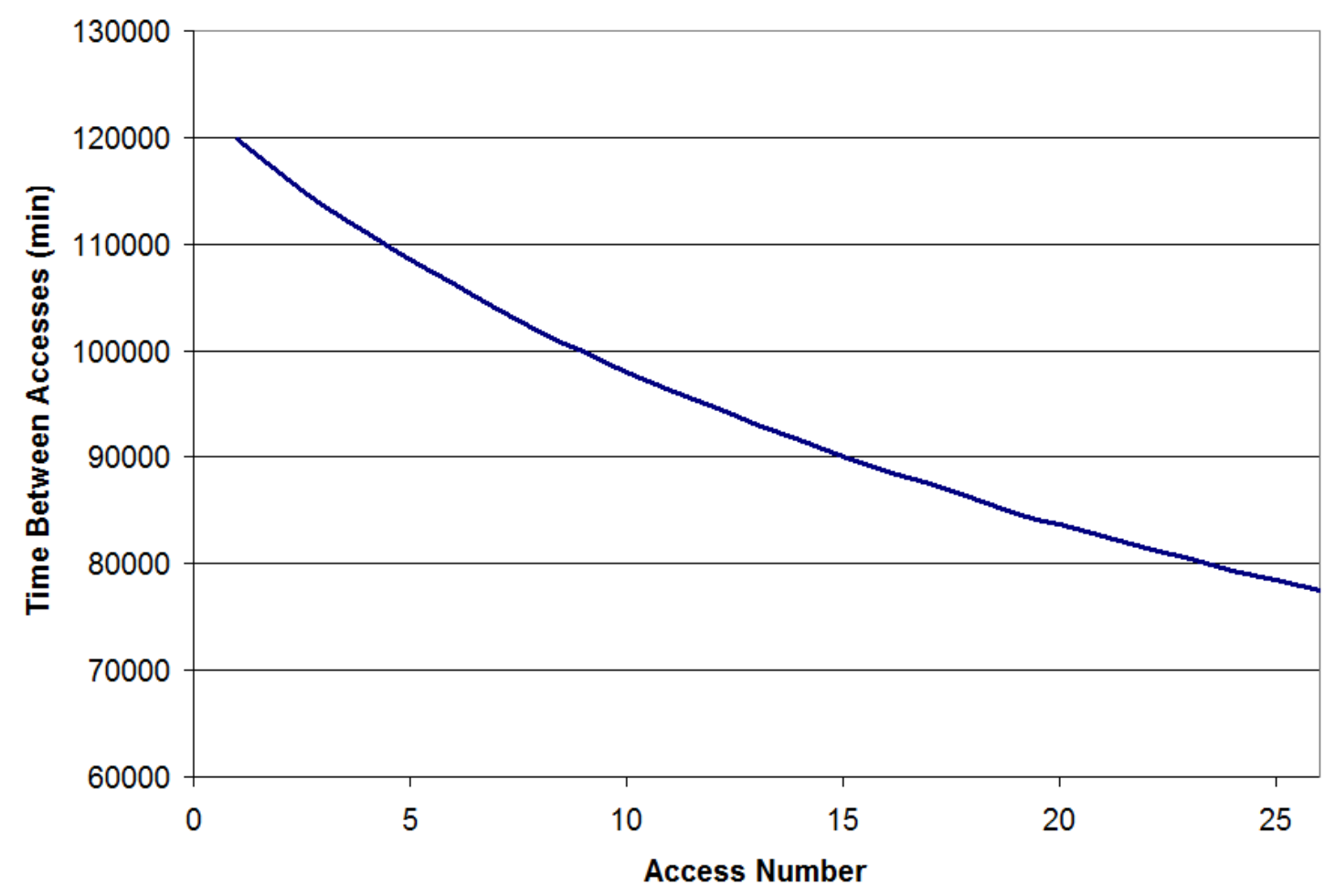

Figure 28: Time between accesses over 5 years CP4 to SAUDICOMSAT_6 (CubeSat Satellites)

\subsection{Correctness}

To check the correctness of the code for the protocol data was taken from a simulation of 365 days and compared to information gathered manually. The areas found where proving correctness was needed are as follows:

\subsubsection{Routing table being populated correctly}

This was by far the easiest to test. Simulations were run with various number of satellites, including one data mule. At the end of the simulation the link state tables were checked against a manual creation of the link state tables. The tables were checked to make sure that every entry that should be there was there and that the entries were correct. 


\begin{tabular}{|c|c|c|c|c|}
\hline Sat 1 & Sat 2 & Acutal & Estimate & Percent \\
\hline CP3 & CP4 & 3047 & 2667 & 12.5 \\
\hline CP3 & MAST & 16269 & 15448 & 5.0 \\
\hline CP3 & SAUDI4 & 949 & 756 & 20.3 \\
\hline CP3 & SAUDI6 & 1588 & 1302 & 18.0 \\
\hline CP4 & MAST & 9583 & 8929 & 6.8 \\
\hline CP4 & SAUDI4 & 3572 & 3229 & 9.6 \\
\hline CP4 & SAUDI6 & 26289 & 25318 & 3.7 \\
\hline MAST & SAUDI4 & 1407 & 1178 & 16.2 \\
\hline MAST & SAUDI6 & 3607 & 3043 & 15.6 \\
\hline SAUDI4 & SAUDI6 & 11060 & 10112 & 8.6 \\
\hline
\end{tabular}

Table 4: Actual Durations vs. Estimated Durations (SAUDI is short for SAUDICOMSAT)

\subsubsection{Routing tables being used correctly to initiate connections}

By creating debug output from the simulation as it runs, the times when connections were being made as well as the steps that need to occur during each connection, were tracked. In the appendix section B a trace of the output is provided that shows one such connection between two satellites that are no longer in discovery mode, but in power save mode.

\subsubsection{Durations of connections are being calculated correctly}

The durations of connections according to the protocol were compared to the actual time that the satellites were in range during the connection. Both of these take into account the grey periods and an error percentage was taken. The average percentage was just under $12 \%$, all of which were under estimating the duration of the links. Underestimating the durations is not necessarily bad. It allows a little extra room if the duration decreases between iterations, or if extra data needs to be sent that wasn't expected. Table 4 shows the exact numbers. Notice that the longer the duration the better the estimate tends to be.

\subsubsection{Shortest Path is computed correctly}

The shortest path was tested for correctness by having the satellites compute shortest paths at different times for different values of data and manually looking through the debug output 
to check that the correct path was computed and the flow was incremented correctly along that path. In addition to this large files that would need to be sent over more than just one path was tested and found to properly distribute the file among several paths.

\subsubsection{Data is transferred correctly}

The transfer of data was tested at the same time as testing the shortest path algorithm by first sending a single small file that barely spanned multiple packets and using the Unix diff command to test that the file being sent was the same as the file that was received at the destination. Once this was done larger files were transmitted on the magnitude of millions of bytes to test that the satellite protocol would be able to handle large files without losing any parts of the files. Once again these large files were compared using the Unix diff command and found to be identical. The final test to make sure data was being transfered correctly and robustly was to send several files (15 files were sent) all of which were $1 \mathrm{MB}$ in size. The comparison of these files showed that even though parts of the files were delivered at different times and took different paths to the destination they still were all transfered correctly.

\subsection{Theoretical Results Using Ideal Connections}

This section shows theoretical results on the effect of different maximum ranges and bit rates of hardware and the effect of adding more data mules and sensors to the network. The calculations were done by simply multiplying the actual duration of each connection by the bit rate over a 365 day period. This information gives good insight into how the network will react with different hardware or as the size of the network grows.

\subsubsection{Average Throughput}

Figure 29 shows the average throughput at different ranges and different speeds for the wireless hardware of one mule. This figure was created by data collected for each satellite 


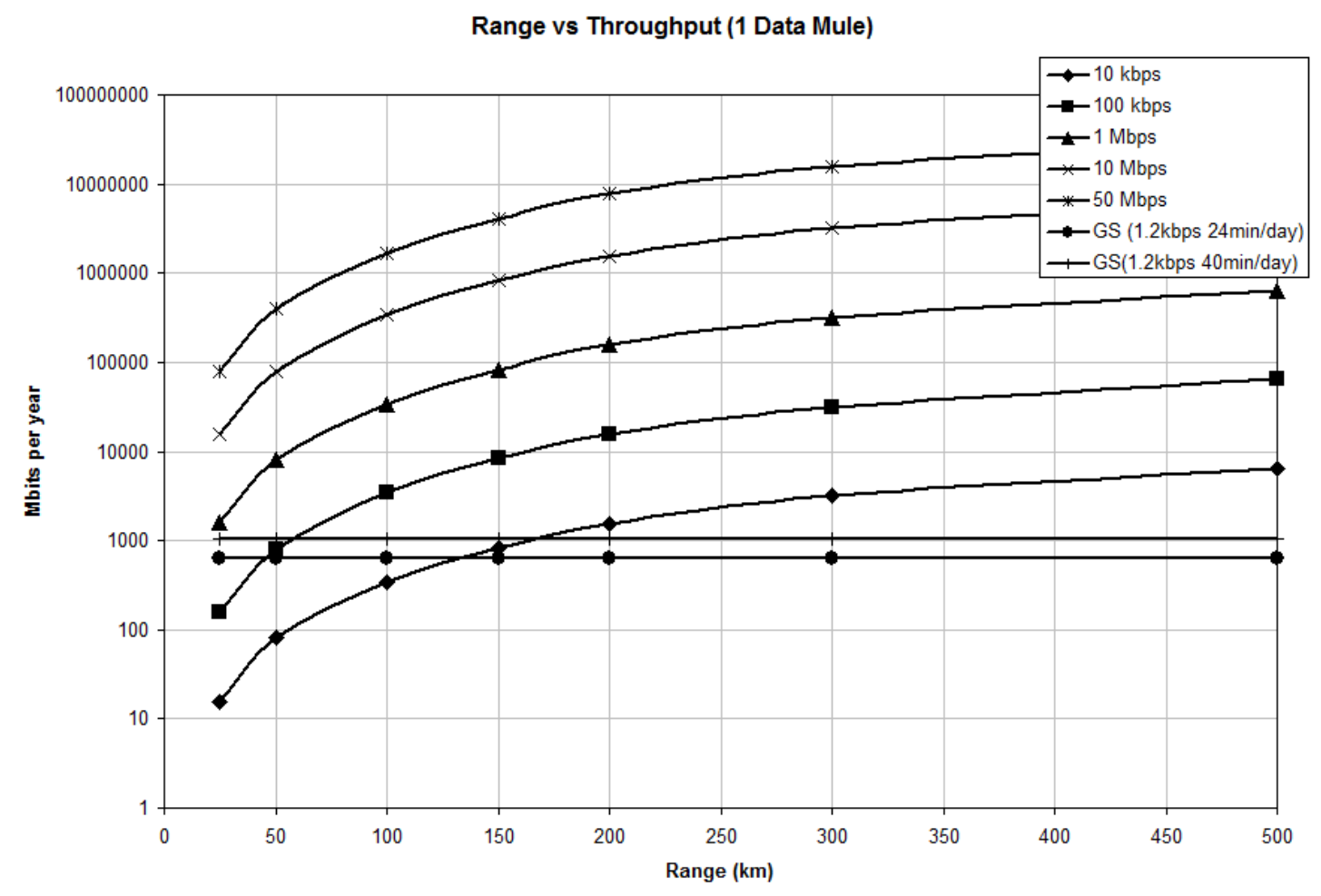

Figure 29: Average throughput over one year with one MULE vs max range of satellites for different bit-rates

communicating directly to the mule and only to the mule. According to the manual calculation of the amount of data able to be processed using the total duration and different bit rates, the fully implemented protocol should easily surpass the throughput of the current implementation.

The average throughput for what is currently in place in the PolySat project is around 1000 megabits or 12.5 megabytes per year as can be seen as the straight horizontal lines in figure 29. What can also be seen in this figure is that even with a 10kbps connection a range of only just over $150 \mathrm{~km}$ is needed to perform better than the current implementation. Therefore if a connection speed of greater than 10kbps is acquired, the range of connection required will be significantly reduced well within a plausible range for current hardware. To allow a cushion for the overhead involved in the connections a more realistic value would be using a 100kbps link, while keeping the range at 150km. 
Figure 30 shows that as the number of mules increase with $150 \mathrm{~km}$ range and $1 \mathrm{Mbps}$ bit rate that the total throughput in one year increases almost linearly. Zero mules in this figure shows the average throughput of a system where the nodes talk to only the ground station as it is currently done for PolySat. This graph shows even more how with just one mule and a range of $150 \mathrm{~km}$ how much throughput is gained. Therefore with only one mule there is a great amount of improvement but even more should be gained by adding more data mules.

To reiterate the values expressed in this section are ideal and assume $100 \%$ utilization of the available bandwidth with no overhead and no lost packets. This information is useful to show the theoretical effects of adding more data mules and more sensors to the network and how different hardware capabilities should affect the maximum throughput.

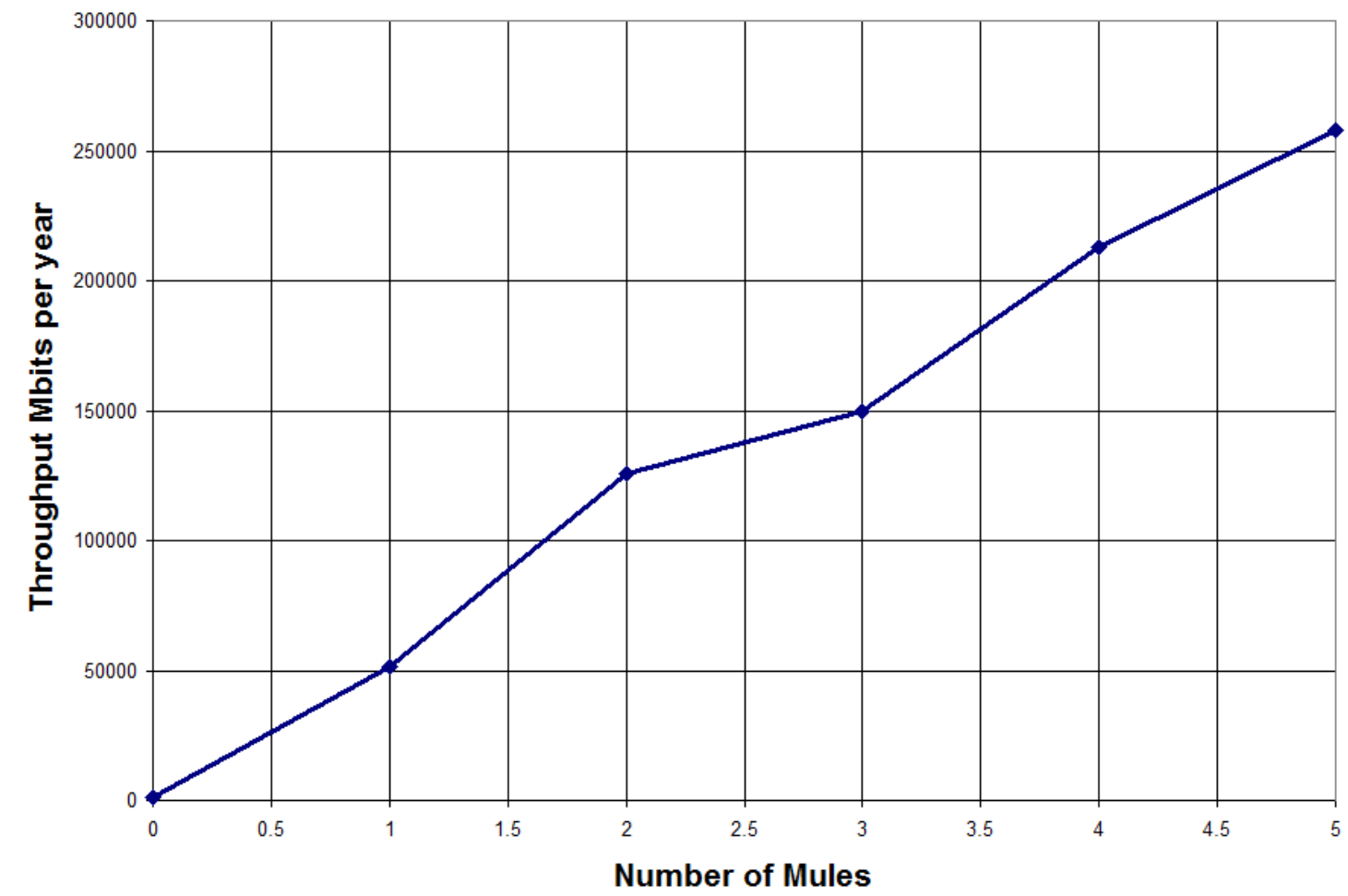

Figure 30: Average throughput over one year vs number of mules at $150 \mathrm{~km}$ and $1 \mathrm{Mbps}$ bit-rate 


\section{Future Work}

\subsection{Simulator}

The simulator created gives a good idea of how the protocol will perform, but is by no means perfect. The ground station side of the simulator is not yet complete, so attempting to measure the data going from the data mules down to the ground stations can not yet be tested and must be left to theoretical evaluation. This is also the reason why the theoretical maximum was used for the current protocol implementation and not an actual number using a protocol. Once this is complete it can also be explored combining the current protocol with the one presented in this paper to limit the end-to-end delay when possible and increase the maximum goodput of the system.

\subsection{Protocol}

As with any new protocol there are many places that can be improved. This one is no exception. Some of these improvements have been mentioned previously in the results section, such as an updated mac protocol and source routing.

The updating of the mac protocol will help deal with bidirectional data better which will allow the protocol to be extended further to use multiple data mules, allow uploading of large amounts of information to the satellites and decrease the collisions already seen in the connections. One such way of implementing a more sophisticated mac protocol would be to make a fully synchronized one that knows exactly how many packets to expect and when to expect them from the satellite that is connected.

Along with this improvement acknowledgments can also be improved in the mac protocol. There are a few ways of doing this, first acks can be appended to the end of every packet that is sent out, assuming there is an acknowledgment that needs to be sent, or there might be a way of XORing the ack packets into the packet being sent. This would have no transmission overhead due to sending acknowledgments, but would have higher overhead 
in reading the information back out of the packet. This would be plausible though because there should only be a few packets that the acknowledgment that is within the packet could be for, it would simply be a matter of trying each one until it was found.

Source routing is important, especially when the network is fully utilized in order to make sure that the intermediate satellites don't try sending the packets they receive to a satellite that the source already plans on providing with the full amount of data that it can handle. This would cause more overhead in the header packets, but would be well worth by the amount of collisions that could be avoided.

Another feature of the satellite network that was noticed when collecting data was that the durations of the connections seem to change in a linear fashion. This could be utilized in order to make better predictions of how much data can be sent between satellites. In the same way the time between connections does change slightly too generally in the same direction, therefore the same linear approximation could be used in order to make better estimations of when the satellites will be in range again. As predictions are found to be correct the amount of buffer space allotted to the satellite can be reduced in order to save more power.

One final improvement to this protocol would be incorporating it with the GENSO [22] project that was described earlier in this paper. If all the ground stations are connected together along with having multiple data mules in space that are shared among all participating CubeSat satellites, the data rates and end-to-end delays achieved would be incredible. 


\section{Conclusion}

The novel protocol proposed in this thesis for connecting a sparse satellite network with the use of data mules shows improvement over the current protocol in use by PolySat. The protocol proposed here also can work together with the other proposed solutions to the problem. Increasing the hardware of the satellites and connecting more ground stations into the network would both increase the performance of this protocol. The current improvements gained are in both maximum throughput and end-to-end delay without sacrificing latency of command and control data. The hardware used in the experiments was well within a reasonable range, only $150 \mathrm{~km}$ range and $100 \mathrm{kbps}$ data rate. The maximum googput was increased by three times when all satellites have large amounts of data to send, and almost five times when only one satellite is sending large amounts of data. The end-to-end delay was almost cut in half when sending $1 \mathrm{MB}$ per day per satellite and is decreased by a factor of at least three when sending large amount of data from only one satellite. With some of the recommended future improvements as well as increased hardware this protocol will outperform the current one by even more. 


\section{References}

[1] Norman Abramson. THE ALOHA SYSTEM: another alternative for computer communications. In Proceedings of the November 17-19, 1970, fall joint computer conference, pages 281-285, Houston, Texas, 1970. ACM.

[2] AGI. Analytical graphics, inc. (AGI), analysis software for land, sea, air, and space. http://www.stk.com/, 2009.

[3] A.F. Ahmed, S.R.V. Ananth, K. Venkateshwaran, V. Vaidehi, and T. Subashri. Multicast security for leo satellite store and forward network. In Signal Processing, Communications and Networking, 2008. ICSCN '08. International Conference on, pages 173-176, 2008.

[4] M.N. Allery and J.W. Ward. The potential for 'store-and-forward' communications using small satellites in low earth orbits. In Satellite Communications - ECSC-3, 1993., 3rd European Conference on, pages 230-235, 1993.

[5] S. Amstutz. A new store and forward message switching system. Communication Technology, IEEE Transactions on, 19(4):528-529, 1971.

[6] S. Bansal, R. Shorey, and A. Misra. Comparing the routing energy overheads of adhoc routing protocols. In Wireless Communications and Networking, 2003. WCNC 2003. 2003 IEEE, volume 2, pages 1155-1161 vol.2, 2003.

[7] CA Cal Poly San Luis Obispo. PolySat. http://polysat.calpoly.edu/index.php, 2009.

[8] G. Comparetto and R. Ramirez. Trends in mobile satellite technology. Computer, 30(2):44-52, 1997.

[9] Thomas Cormen, Charles Leiserson, Ronald Rivest, and Clifford Stein. pages 651663. McGraw-Hill Book Company, 2nd edition edition, 2001.

[10] H. Cruz-Sanchez, L. Franck, and A.-L. Beylot. Precomputed routing in a store and forward satellite constellation. In Vehicular Technology Conference, 2007. VTC-2007 Fall. 2007 IEEE 66th, pages 240-243, 2007.

[11] N. Hamamoto, Y. Arimoto, Y. Hashimoto, T. Ide, and M. Sakasai. High speed and global store and forward communication system using LEO satellites. In Universal Personal Communications, 1994. Record., 1994 Third Annual International Conference on, pages 418-422, 1994.

[12] Yan He and S. Pelagatti. CRT: an adaptive routing protocol for LEO satellite networks. In Information and Communication Technologies, 2006. ICTTA '06. 2nd, volume 2, pages 2496-2501, 2006.

[13] Qijie Huang, Boon Sain Yeo, and Peng-Yong Kong. A routing algorithm to provide end-to-end delay guarantee in low earth orbit satellite networks. In Vehicular Technology Conference, 2004. VTC 2004-Spring. 2004 IEEE 59th, volume 5, pages 2911-2915 Vol.5, 2004. 
[14] Qijie Huang, Boon Sain Yeo, and Peng-Yong Kong. An enhanced QoS routing algorithm for provision of end-to-end delay guarantee in low earth orbit satellite networks. In Wireless Communications and Networking Conference, 2005 IEEE, volume 3, pages 1485-1490 Vol. 3, 2005.

[15] Anthony Hung, Marie-Jose Montpetit, and George Kesidis. ATM via satellite: a framework and implementation. Wirel. Netw., 4(2):141-153, 1998.

[16] Philo Juang, Margaret Martonosi, Daniel Rubenstein, Hidekazu Oki, Li-Shiuan Peh, and Yong Wang. Energy efficient computing for wildlife tracking: Design tradeoffs and early experiences with ZebraNet. In 2002 International Conference on Architectural Support for Programming Languages and Operating Systems - ASPLOS, pages $96-107,2002$.

[17] O. Korcak and F. Alagoz. Analysis of priority-based adaptive routing in satellite networks. In Wireless Communication Systems, 2005. 2nd International Symposium on, pages 629-633, 2005.

[18] John Magliacane. PREDICT - a satellite Tracking/Orbital prediction program. http://www.qsl.net/kd2bd/predict.html, 2009.

[19] N2YO.com. LIVE REAL TIME SATELLITE AND SPACE SHUTTLE TRACKING. http://www.n2yo.com/, 2009.

[20] CalPoly San Luis Obispo. CubeSat community website - home. http://cubesat.calpoly.edu/, February 2009.

[21] GEO ORBIT. Footprints by dish size - definition of geostationary (Geosynchronous), polar, LEO, HEO, MEO, sun synchronous orbits, brief satellite history. http://www.geo-orbit.org/sizepgs/geodef.html, 2009.

[22] GENSO Project. GENSO. http://www.genso.org/, 2009.

[23] S.D. Shapiro. Generalizations of random store and forward communication networks. Proceedings of the IEEE, 55(12):2191-2192, 1967.

[24] K. Sidibeh and T. Vladimirova. Wireless communication in LEO satellite formations. In Adaptive Hardware and Systems, 2008. AHS '08. NASA/ESA Conference on, pages 255-262, 2008.

[25] Network Simulator. The network simulator - ns-2. http://www.isi.edu/nsnam/ns/, 2009.

[26] J.W. Ward. Store-and-forward message switching using polar-orbiting microsatellites. In Message Handling - Past, Present and Future, IEE Colloquium on, pages 9/1-9/4, 1991. 
[27] Bin Zhang, G.S. Sukhatme, and A.A. Requicha. Adaptive sampling for marine microorganism monitoring. In Intelligent Robots and Systems, 2004. (IROS 2004). Proceedings. 2004 IEEE/RSJ International Conference on, volume 2, pages 1115-1122 vol.2, 2004.

[28] Zhi-Gang Zhao. A robust ATM-Based routing algorithm in LEO satellite communication networks. In Innovative Computing, Information and Control, 2007. ICICIC '07. Second International Conference on, page 626, 2007. 


\section{Appendices}

\section{A Packet Structures}

\section{A.0.1 MAC Layer Header Packet}

\begin{tabular}{|c|c|c|c|c|c|c|c|}
\hline 0 & 1 & 2 & 3 & 4 & 5 & 6 & 7 \\
\hline Version & Padding & Length & Packet Type & Checksum \\
\hline \multicolumn{3}{|c|}{ Source Address } & \multicolumn{3}{c|}{ Destination Address } \\
\hline \multicolumn{3}{|c|}{ Packet ID } & \multicolumn{3}{c|}{ Data $\ldots}$. \\
\hline
\end{tabular}

Figure 31: Mac Header Packet

The header packet is attached to all transmissions from every sensor satellite and all mules. This serves as a common ground that all nodes in the network can use to decipher an incoming message. The fields contained in the header packet are designed to make this header extensible and versioned so it can be updated at a later time. The fields in the 20 byte header are defined as follows:

- Version

- This version field allows the header to be replaced with a new version at a later time if it ever needs to be updated. The current version number is 1 .

- Padding

- Padded with 0's in order to make the packet size a multiple of two.

- Length

- This is a two byte number that signifies the total length of the packet.

- Packet Type

- This field is a two byte integer that tells the receiver what the data field contains. The current values for the next header are:

* ACK/NACK

* Neighbor Request

* Neighbor Reply

* Hello Packet

* You There Packet

* Data Request

* END connection

* Higher Level Protocol 
- Checksum

- The checksum field is a checksum of the entire packet that allows the satellite to make sure that the packet is free of errors.

- Source Address

- The source address is the ID of the satellite that sent this packet.

- Destination Address

- The destination address is the ID of the satellite that is meant to receive the packet.

- Packet ID

- This is an ID for the packet to can keep track of what packets get delivered and what packets don't get delivered.

- Data

- This is a segment of a variable length that could contain data of any of the next header types.

\begin{tabular}{|c|c|c|c|c|c|c|c|}
\hline 0 & 1 & 2 & 3 & 4 & 5 & 6 & 7 \\
\hline \multicolumn{4}{|c|}{ Satellite 1 Address } & \multicolumn{4}{|c|}{ Satellite 2 Address } \\
\hline \multicolumn{2}{|c|}{ Sat 1 Type } & \multicolumn{2}{|c|}{ Sat 2 Type } & \multicolumn{4}{|c|}{ Time of last Connection } \\
\hline \multicolumn{4}{|c|}{ Time until next Connect } & \multicolumn{4}{|c|}{ Duration of last Connect } \\
\hline
\end{tabular}

Figure 32: Neighbor Entry

The neighbor reply packet consists of a header packet with the next header field assigned as neighbor reply, then a two byte short, called N, of how many neighbor entries there are inside this packet, another two byte short signifying the total number of neighbor entries that will be sent and finally the series of $\mathrm{N}$ neighbor entries which are depicted in Figure 32. The total number of neighbors is required to make sure the satellite receives all of the entries needed and none of the packets are lost. Each neighbor entry is 26 bytes long consisting of 8 different fields. The fields are as follows:

- Satellite 1 Address

- ID of one of the satellites in the meeting. 
- Satellite 2 Address

- ID of the second satellites in the meeting. If this value is the same as Satellite 1 then this is an entry directly from that satellite and the time of last meeting should be gotten from the current clock value and the time until next meeting should be calculated.

- This is so each satellite can transmit its own type and ID along with the normal neighbor replies and eases the implementation of updating the link state information when two satellites come into contact.

- Satellite 1 Type

- The type of satellite 1

- Satellite 2 Type

- The type of satellite 2

- Time Last Seen

- Number of seconds since epoch that the last meeting of the two satellites occurred.

- Time Between last Two Sightings

- Seconds between the last two sightings of that satellite, which can be used to estimate the time of the next sighting.

- If this value is 0 then the Time until next meeting has not yet been established.

- Duration

- This is the duration of the last connection between these two satellites. This is used to compute the amount of data which can be transferred between the two.

- Bit rate

- The bit rate achieved between these two satellites. This is used to compute the amount of data which can be transferred between the two.

\section{A.0.2 ACK/NACK Packet}

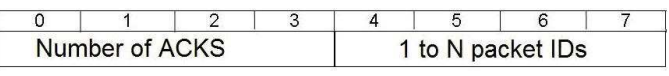

Figure 33: Acknowledgment Packet 
The payload of an acknowledgment packet is simply the number of packets being acknowledged in the packet followed by the packet IDs of that many packets. Currently only one packet ID is contained in an acknowledgment but this setup will allow this to be optimized at a later date.

\section{A.0.3 Network Layer Header}

\begin{tabular}{|c|c|c|c|c|c|c|c|}
\hline 0 & 1 & 2 & 3 & 4 & 5 & 6 & 7 \\
\hline Version & $T \mathrm{~L}$ & Next Header & \multicolumn{5}{c|}{ Source Address } \\
\hline \multicolumn{3}{|c|}{ Destination Address } & \multicolumn{3}{c|}{ DATA / Next Header... } \\
\hline
\end{tabular}

Figure 34: Network Layer Header

The network layer header is used to send packets from one satellite to another using possibly multiple hops. The MAC layer header allows only single hop sending of packets, but with this header information can be forwarded among the satellites allowing this to be a multi-hop network.

- Version

- Version field to allow different versions to be created. (current version is 1)

- TTL

- Time To Live of the packet to make sure it doesn't get stuck in a loop.

- Next Header

- Specifies what is contained in the Data Section.

- Currently only data or instruction packet

- Source Address

- The original source of this packet.

- Destination Address

- The final destination of this packet 


\begin{tabular}{|l|l|l|l|l|l|l|l|}
\hline 0 & 1 & 2 & 3 & 4 & 5 & 6 & 7 \\
\hline \multicolumn{3}{|c|}{ File ID } & \multicolumn{3}{|c|}{ Segment Number } \\
\hline \multicolumn{3}{|c|}{ Segment Size } & \multicolumn{3}{|c}{ Data .... } \\
\hline
\end{tabular}

Figure 35: Data Packet

\section{A.0.4 Transport Layer Data Packet}

The data packet has many specific requirements which had to be overcome during its design. The source address from the Network Layer header is used in combination with the File ID to uniquely identify every piece of data being transferred through the network. The segment size specifies the amount of data that is sent in each packet, except for the final segment which may be smaller. The actual amount of data that is contained in the packet is propagated up from the original length of the packet that is specified in the MAC header, so is knows when the final packet of a file is reached.

- File ID

- Unique file ID from the source address.

- This file ID is a sequential number starting at 1 to allow the sink to keep track of what files it is missing.

- File Sequence Number

- This is a sequence number used to put the packets back in the correct order, it is especially necessary in a satellite environment because packets will arrive out of order most of the time.

- Segment Size

- This is the segment size that is contained in each packet (except for the final segment which may be smaller)

\section{A.0.5 Transport Layer Instruction Packet}

\begin{tabular}{|l|c|c|c|c|c|c|c|}
\hline 0 & 1 & 2 & 3 & 4 & 5 & 6 & 7 \\
\hline \multicolumn{3}{|c|}{ Instruction ID } & & \multicolumn{3}{|c|}{0 to $\mathrm{N}$ parameters... } \\
\hline
\end{tabular}

Figure 36: Instruction Packet 
The instruction packet is used to send control information to the sensors or mules usually originating from the ground station, but not limited to this. No instructions are specified as of yet, but these could be used to communicate with the applications installed on the sensors or the OS.

- Instruction ID

- ID of the instruction to perform

- These will usually originate from the ground station as control information for the sensors.

- Parameters

- These are the parameters for the given instruction.

- Each instruction must have a constant known number of parameters.

\section{B Example Simulation Output}

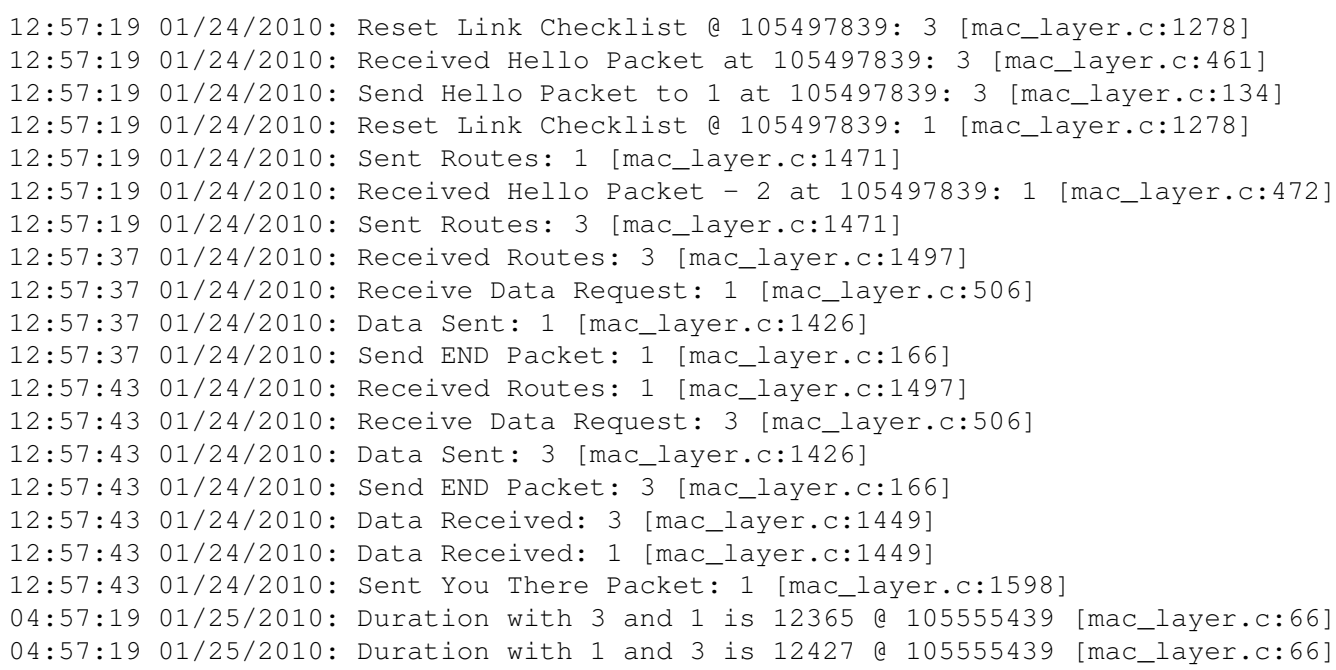

\section{Example Simulation Definition File}

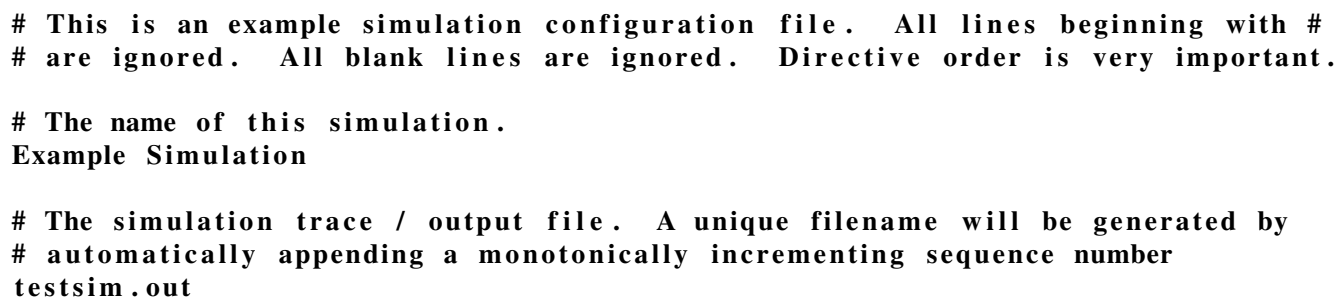




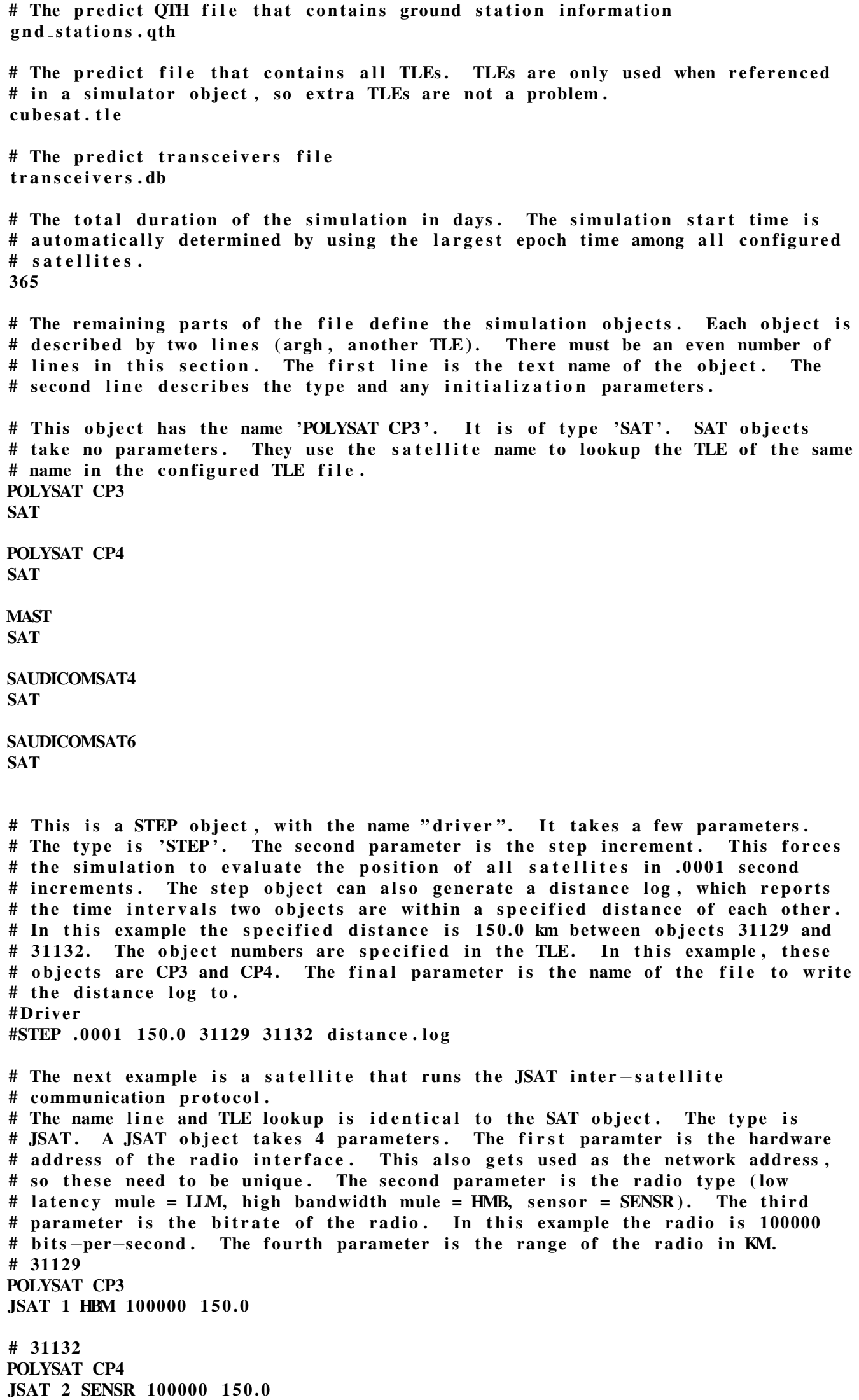


\# 31126

MAST

JSAT 3 SENSR 100000150.0

\# 31127

SAUDICOMSAT4

JSAT 4 SENSR 100000150.0

\# 31121

SAUDICOMSAT6

JSAT 5 SENSR 100000150.0

\section{Header Files}

\section{D.1 Satellite}

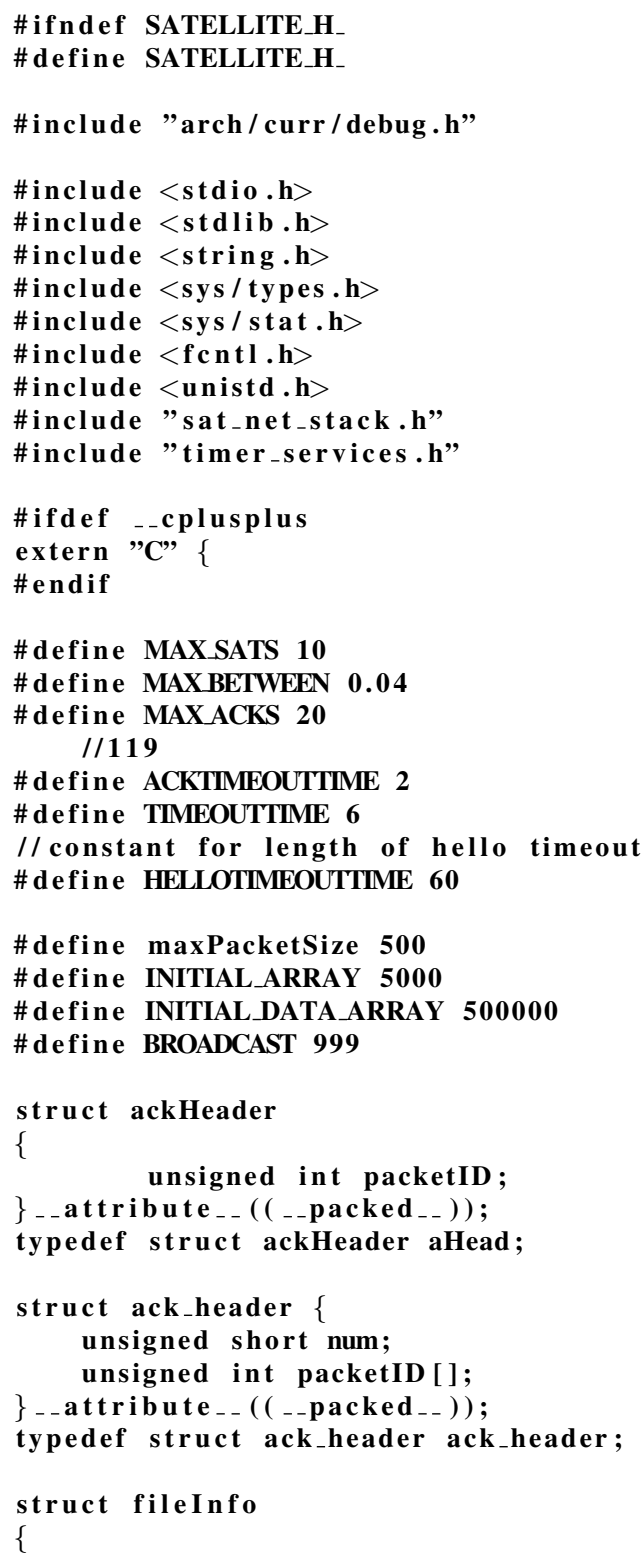




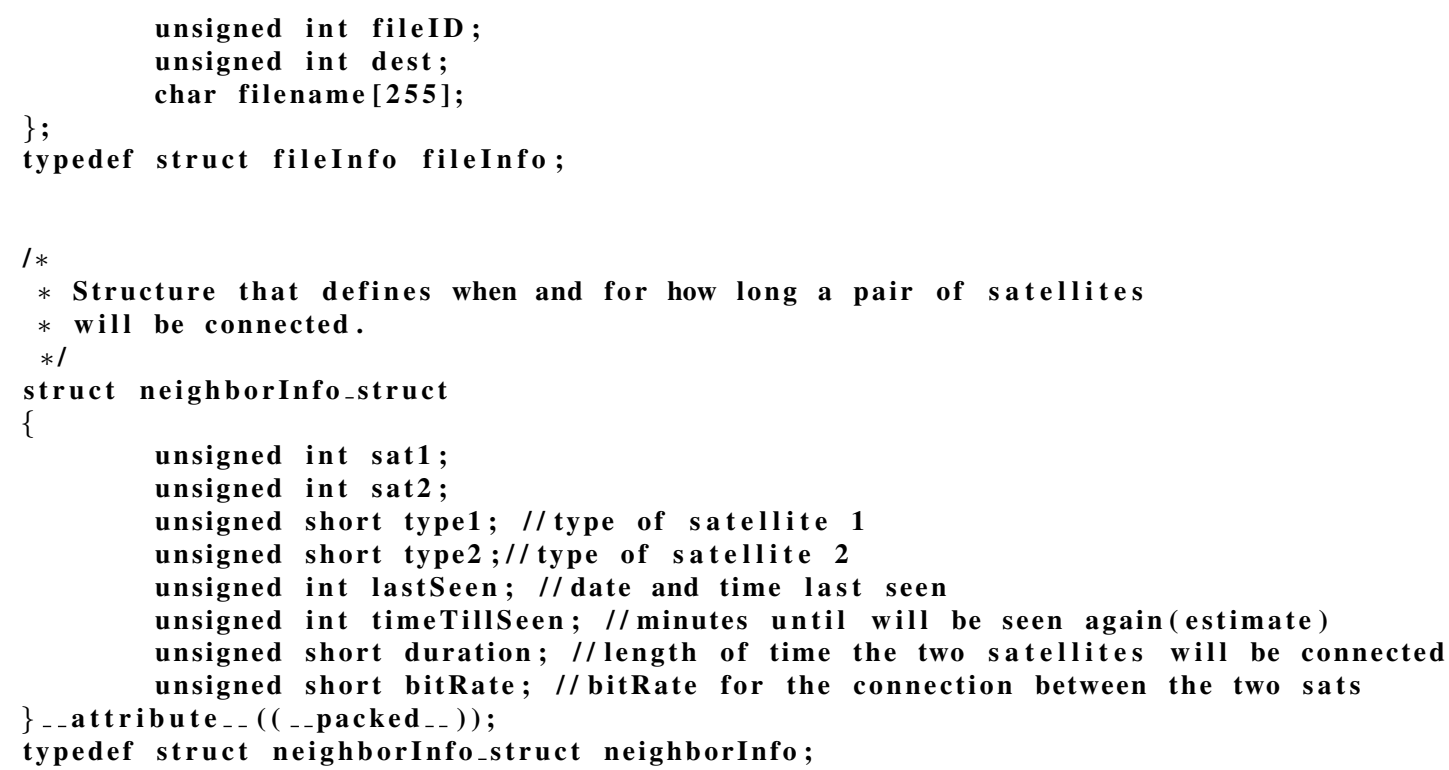




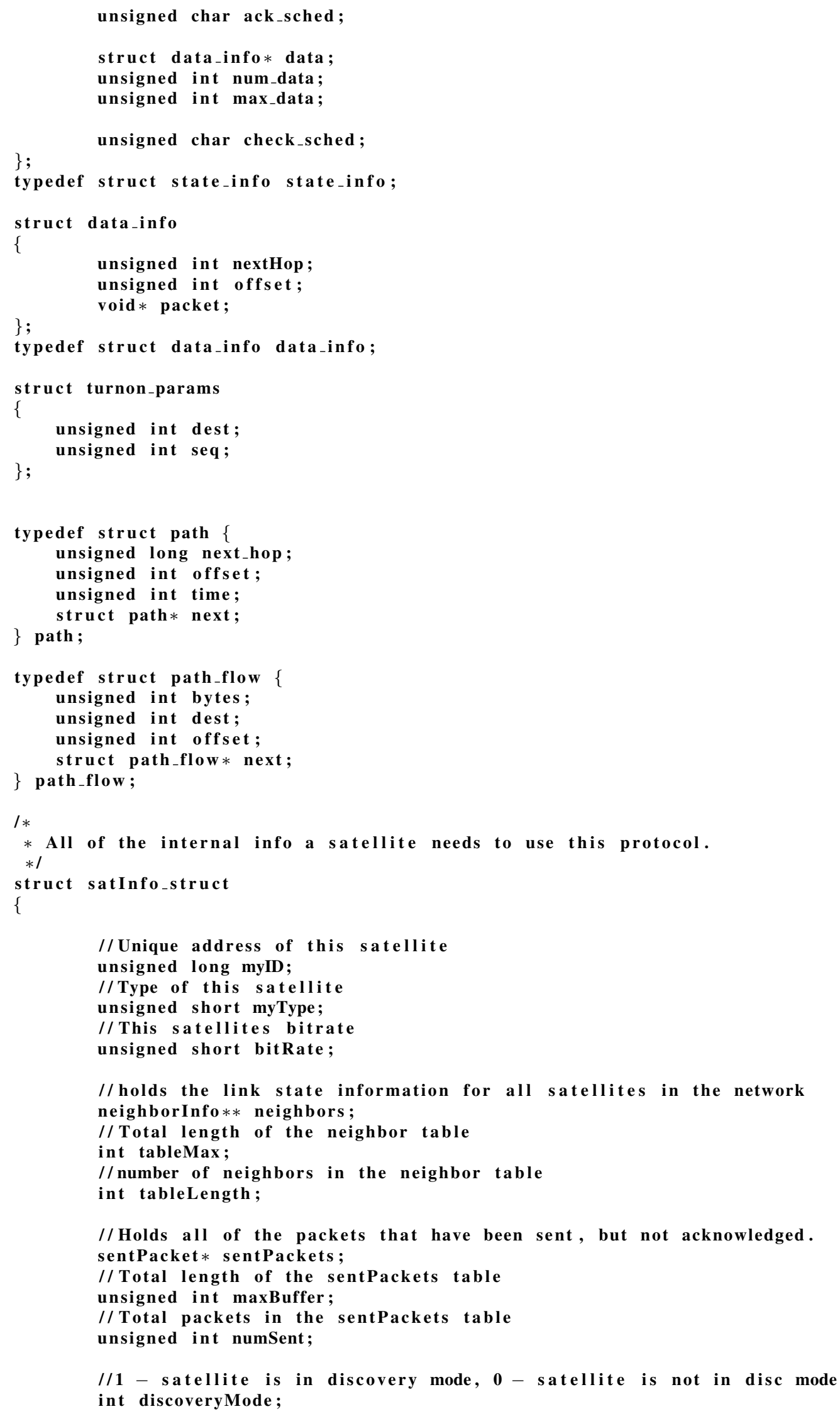




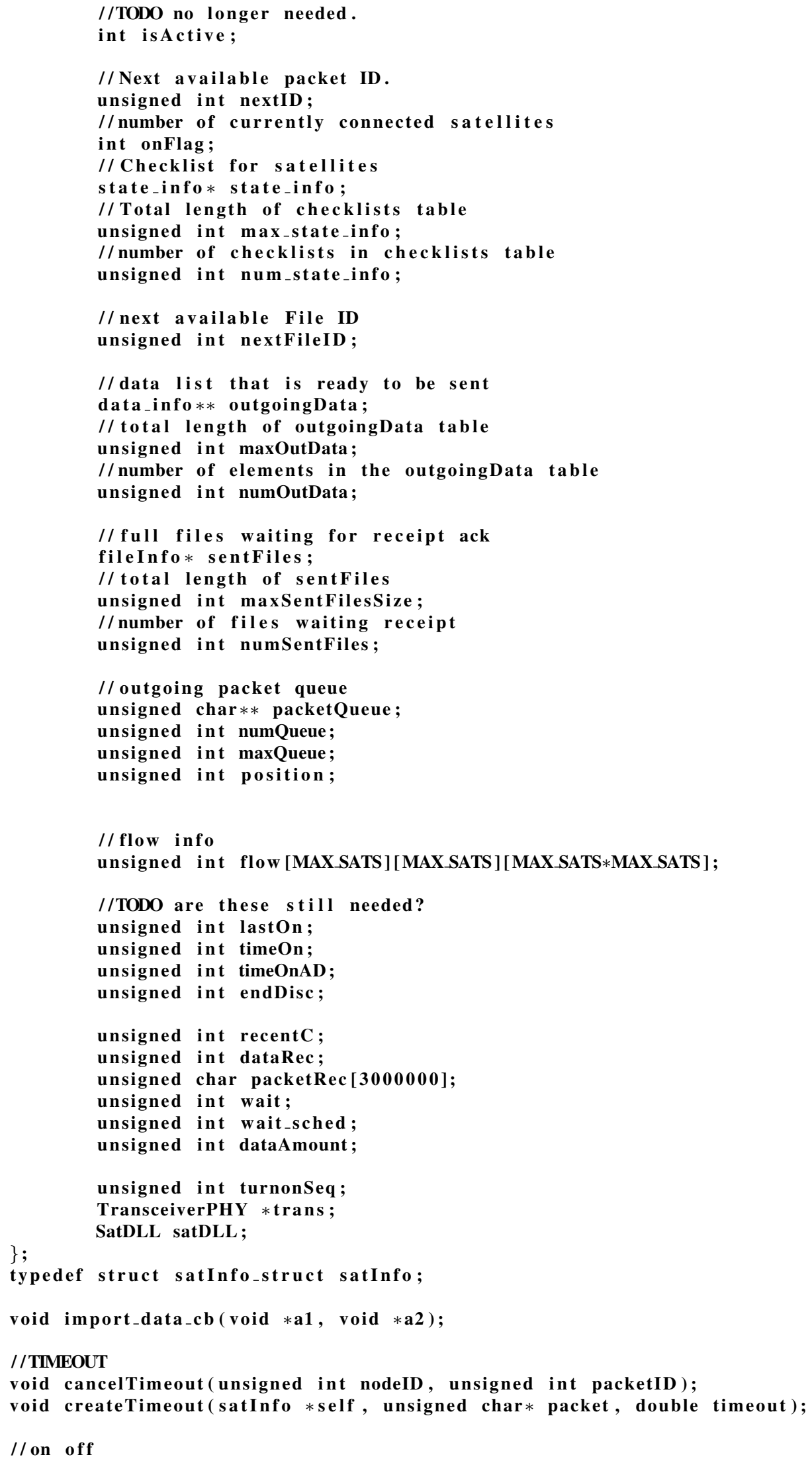




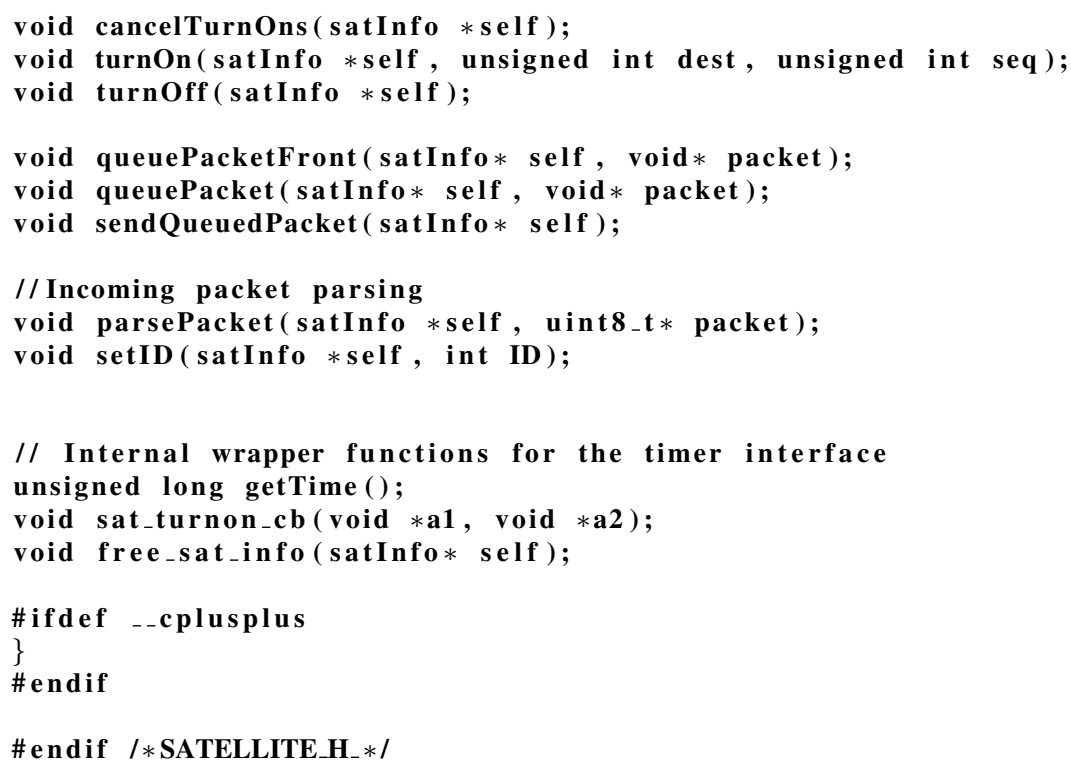

\section{D.2 Mac Layer}

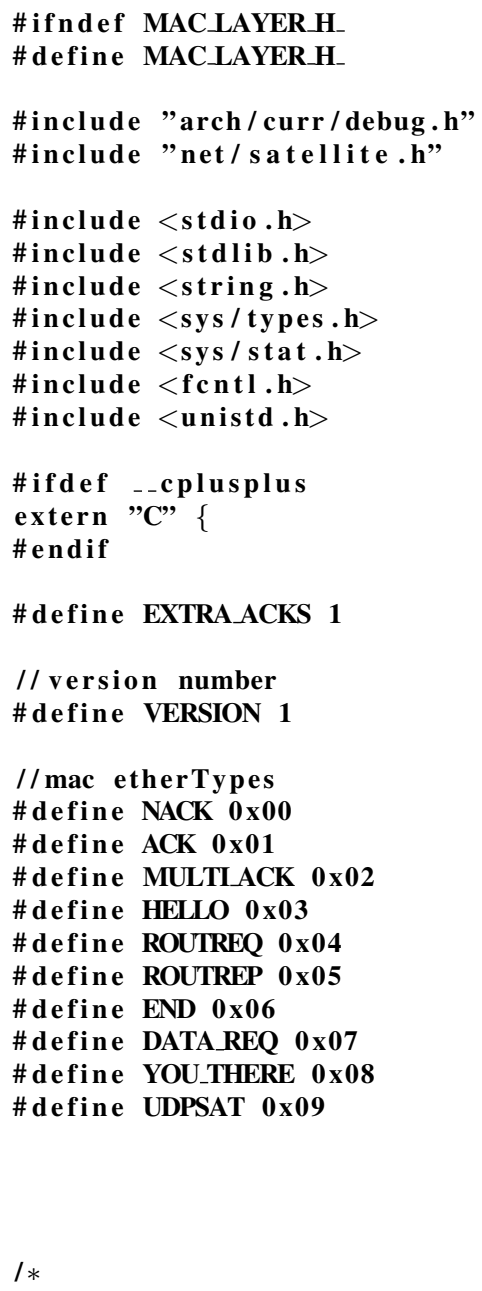




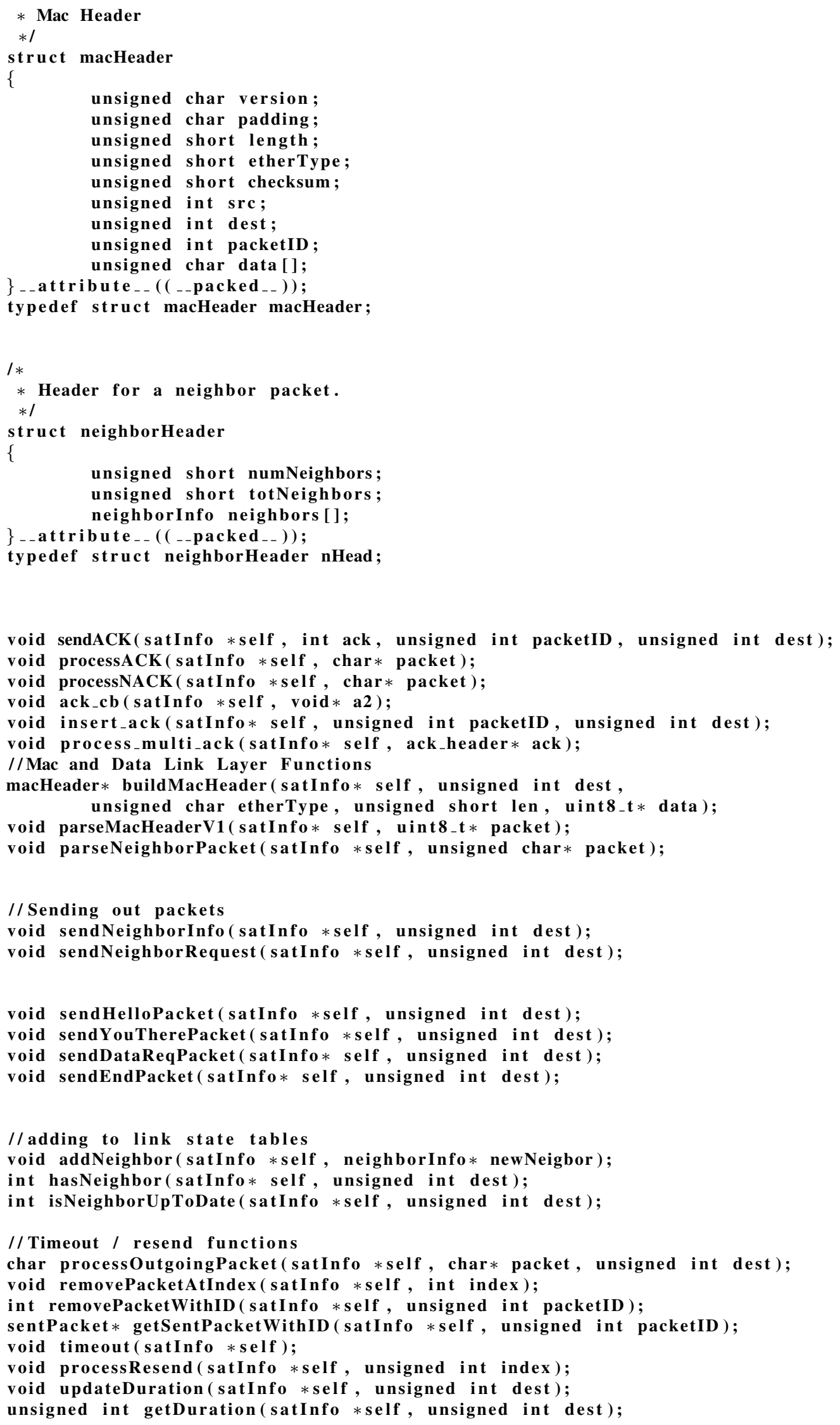




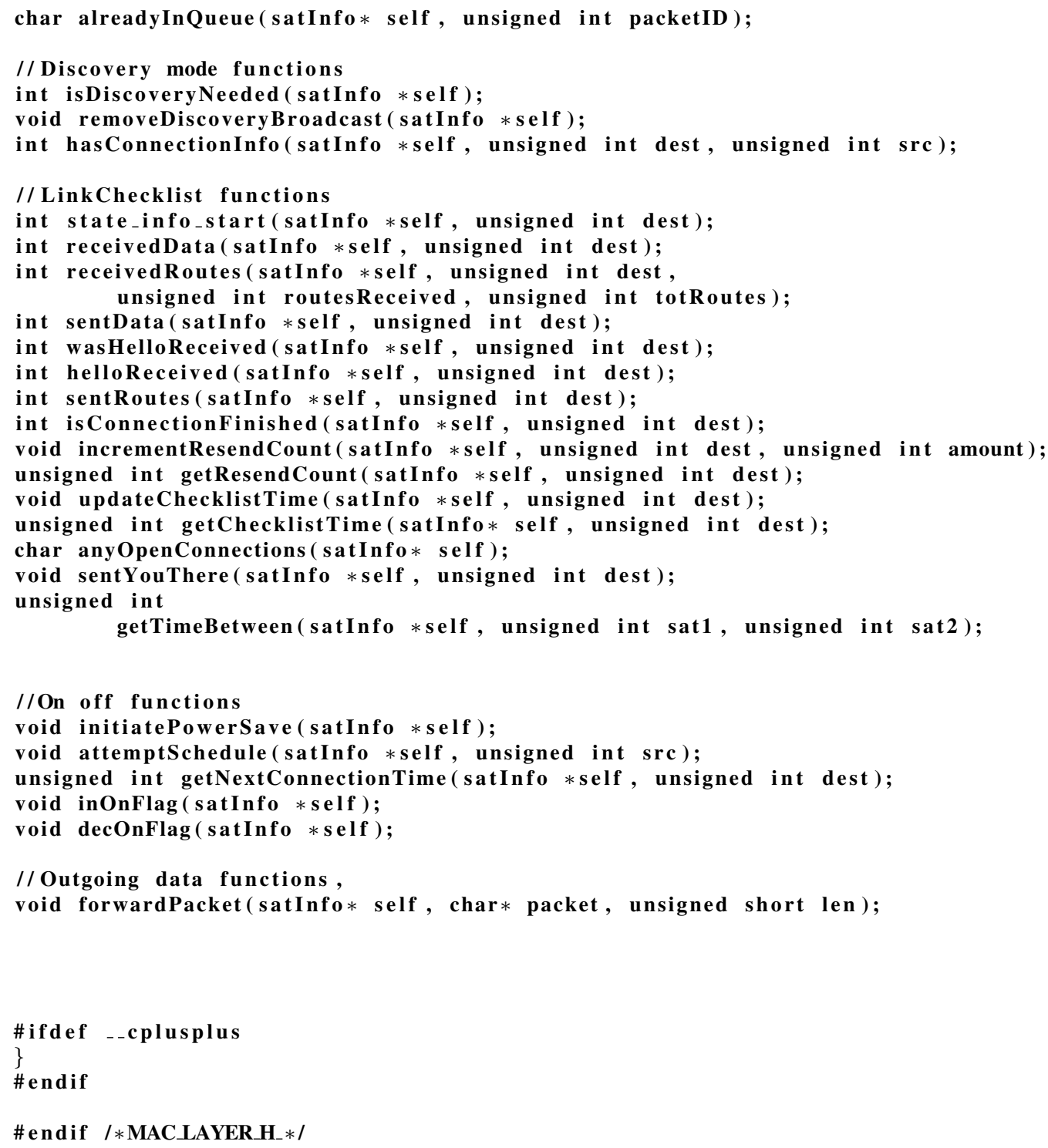

\section{D.3 Network Layer}

\# if nd ef NETWORK_LAYER_H \#d efine NETWORK_LAYER_H_

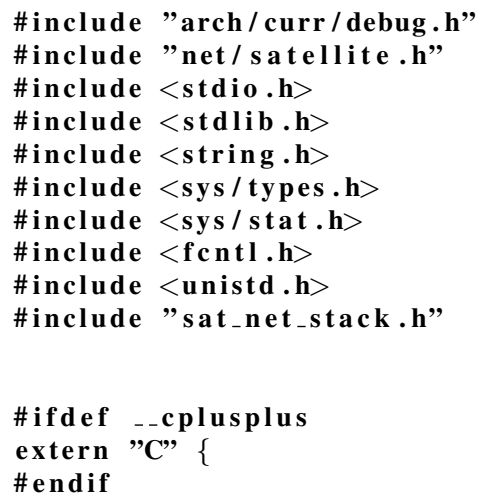




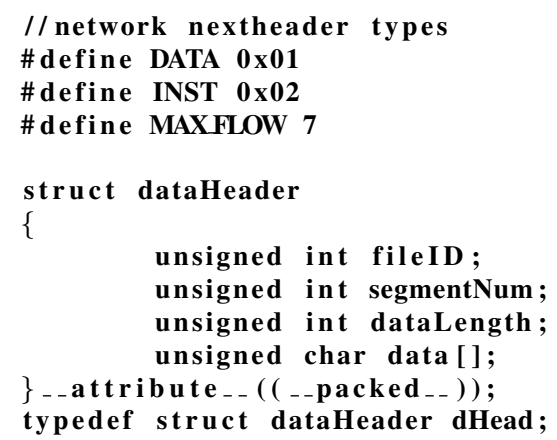

path $*$ dfs_time ( $\operatorname{satInfo} *$ self, unsigned int src, unsigned int dest, unsigned int curTime); path $*$ dfs_main ( $\operatorname{satInfo*}$ self, unsigned int src, unsigned int dest, unsigned int curTime, unsigned int avoid); 


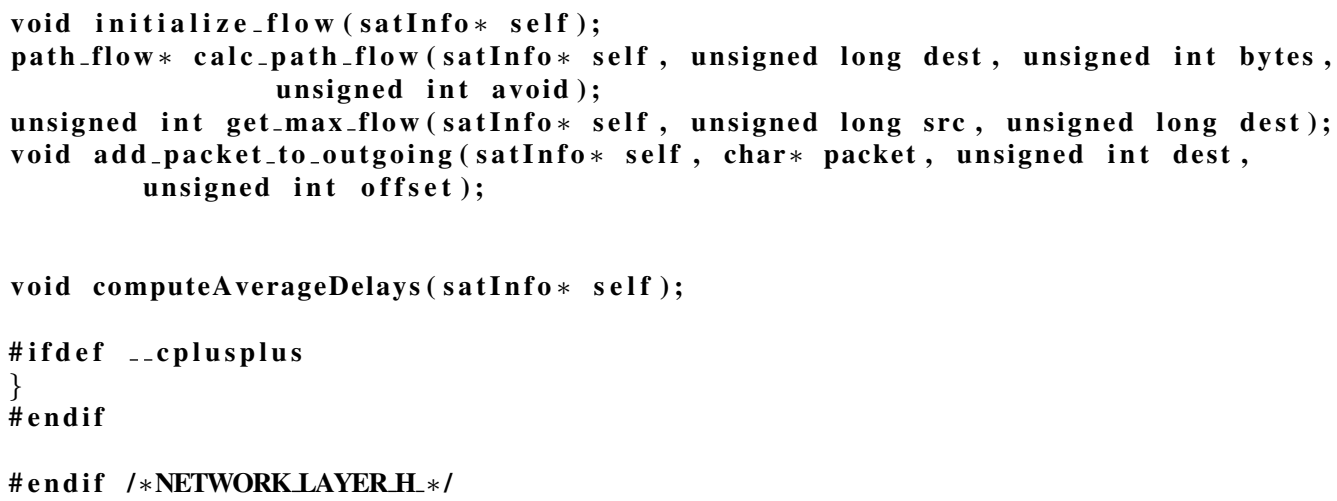

\section{E Select Source Code}

\section{E.1 Scheduling connection}

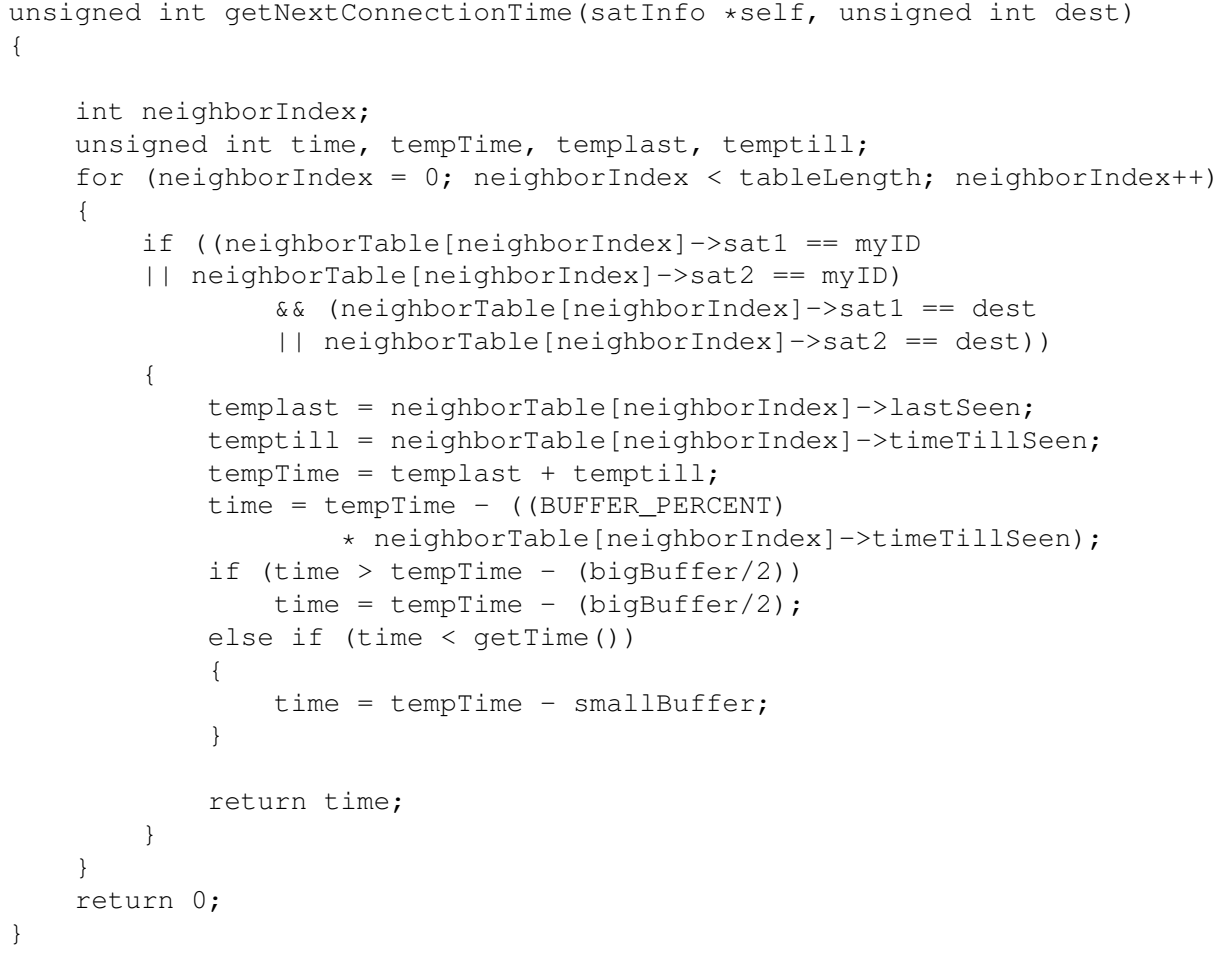

\section{E.2 Shortest Path}

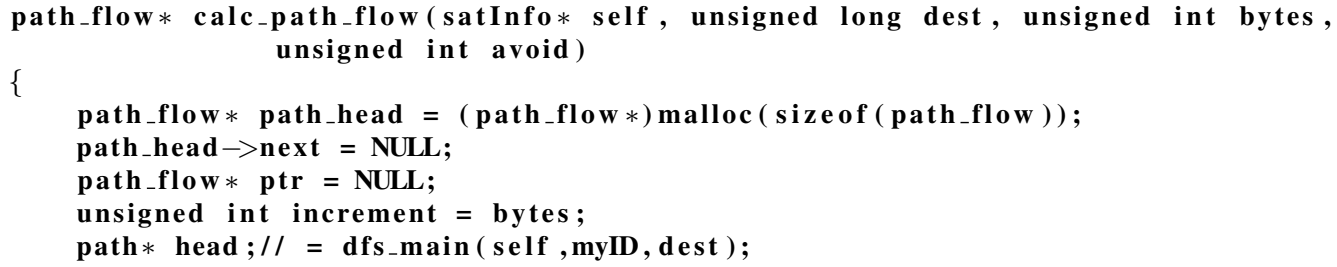




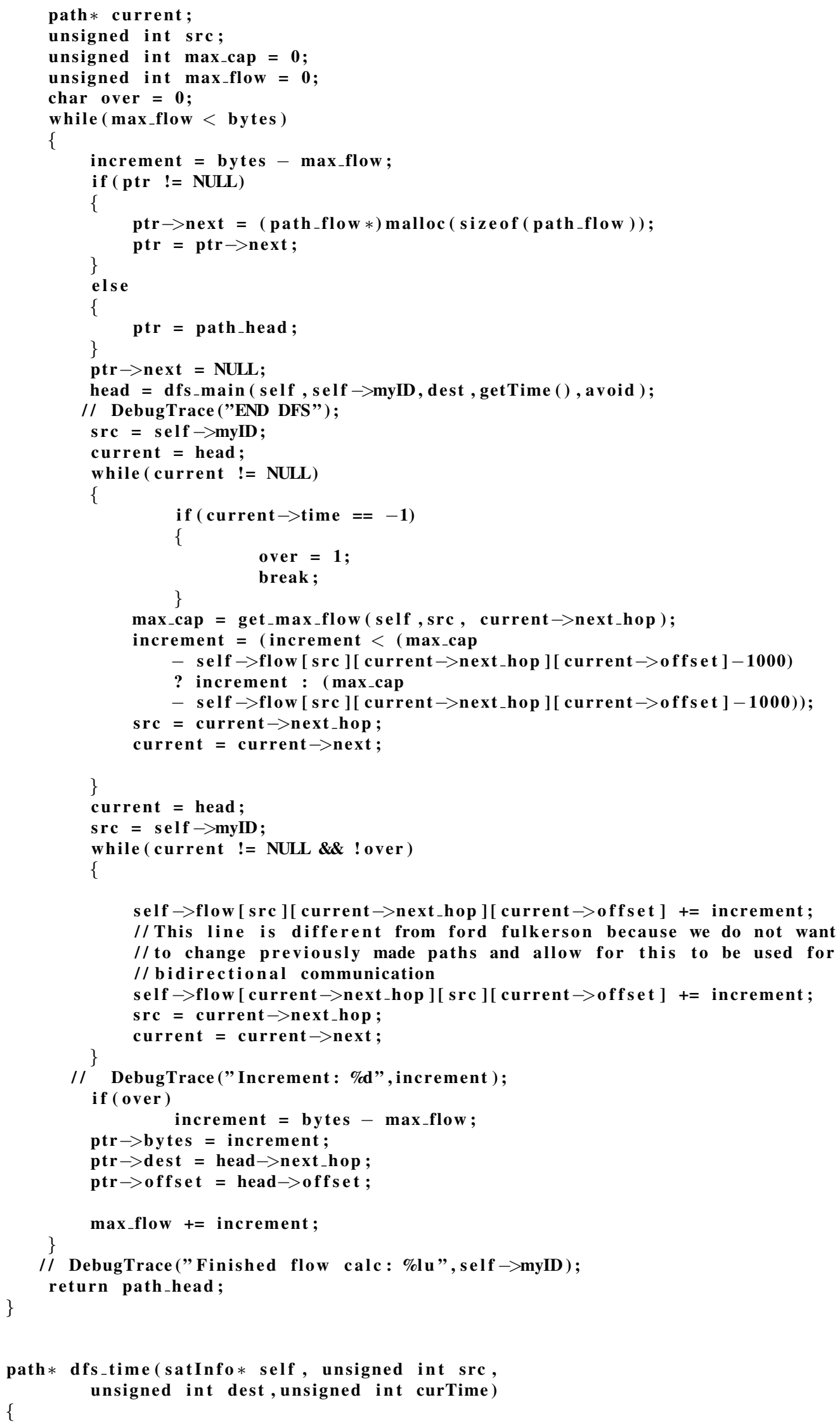




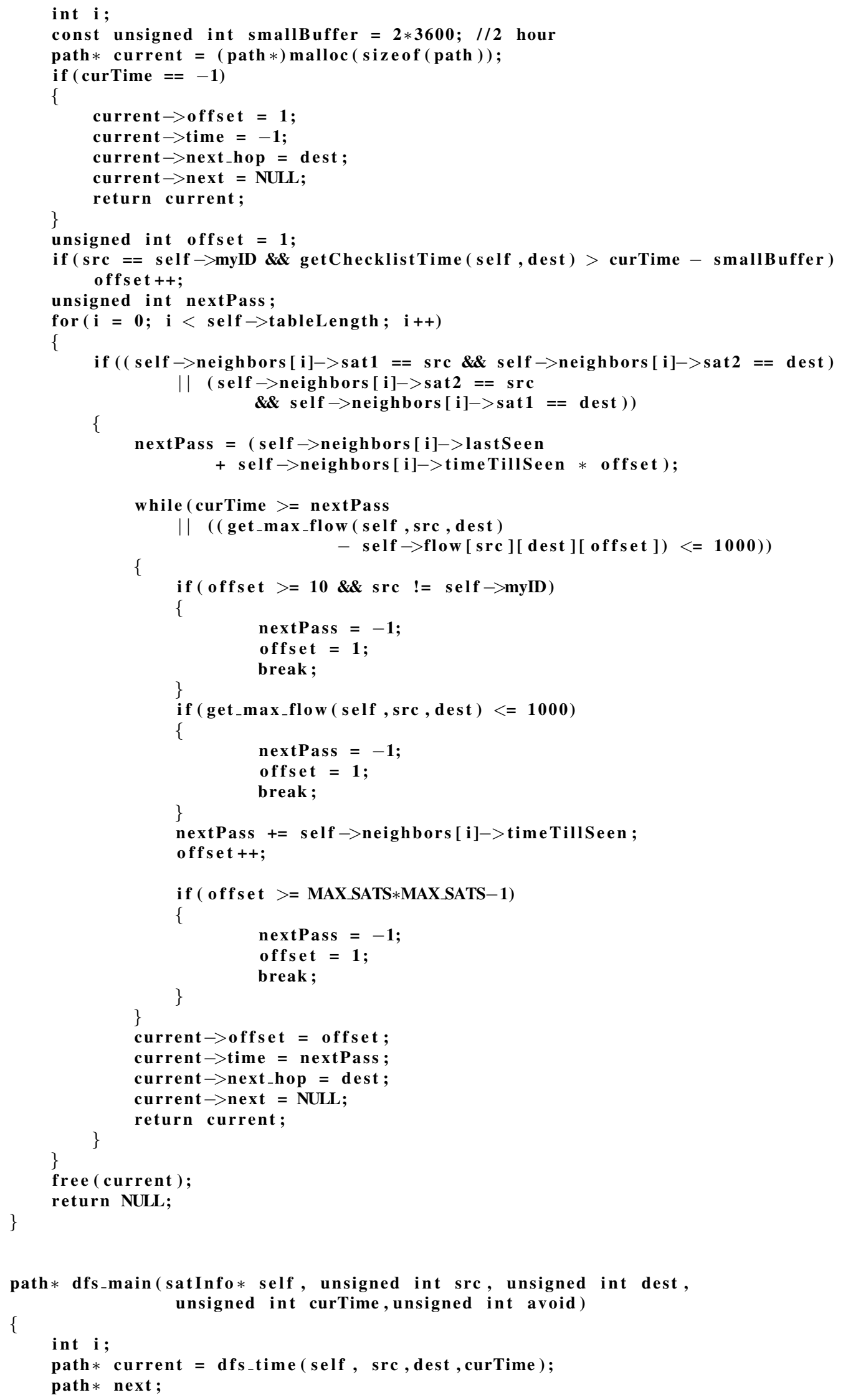




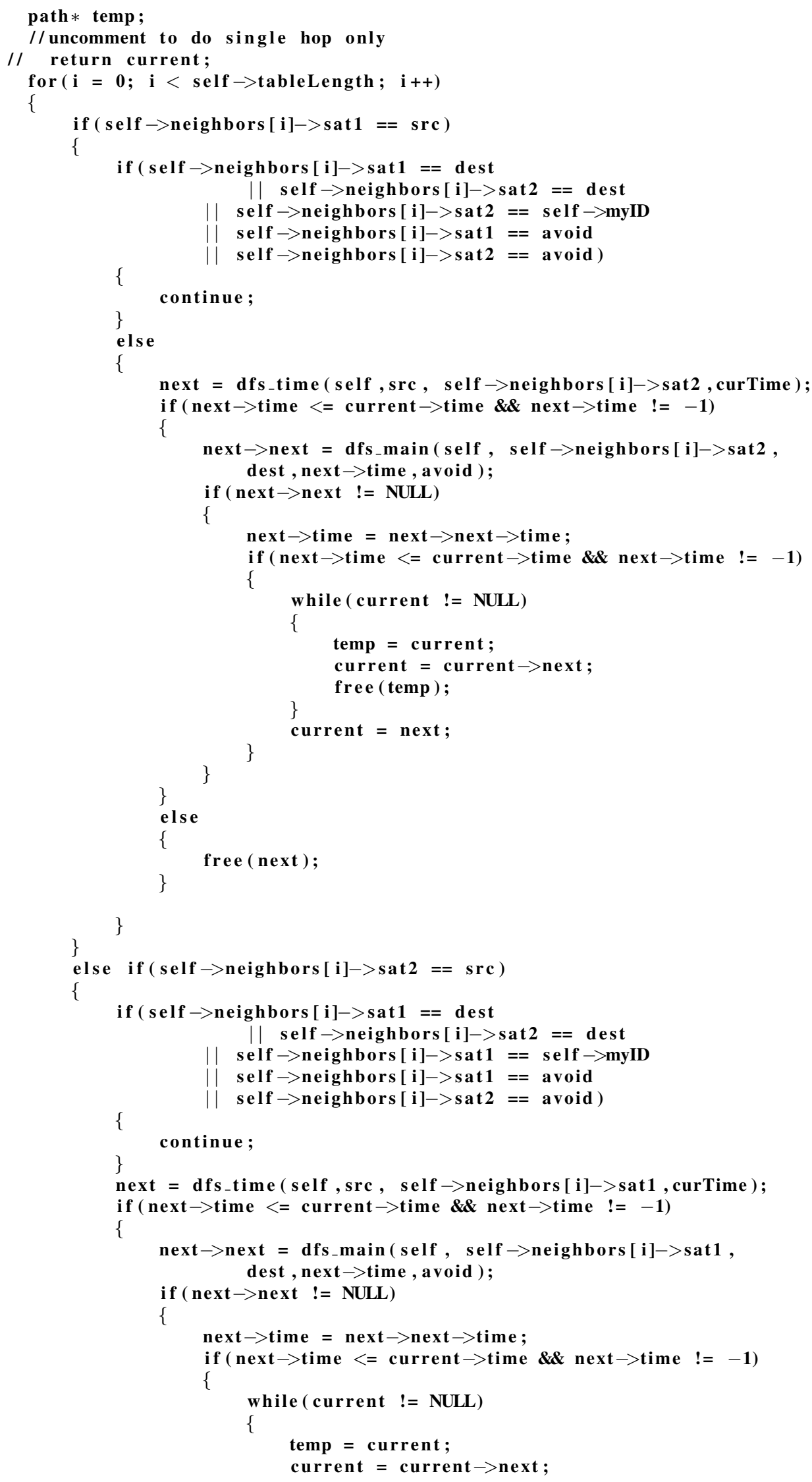




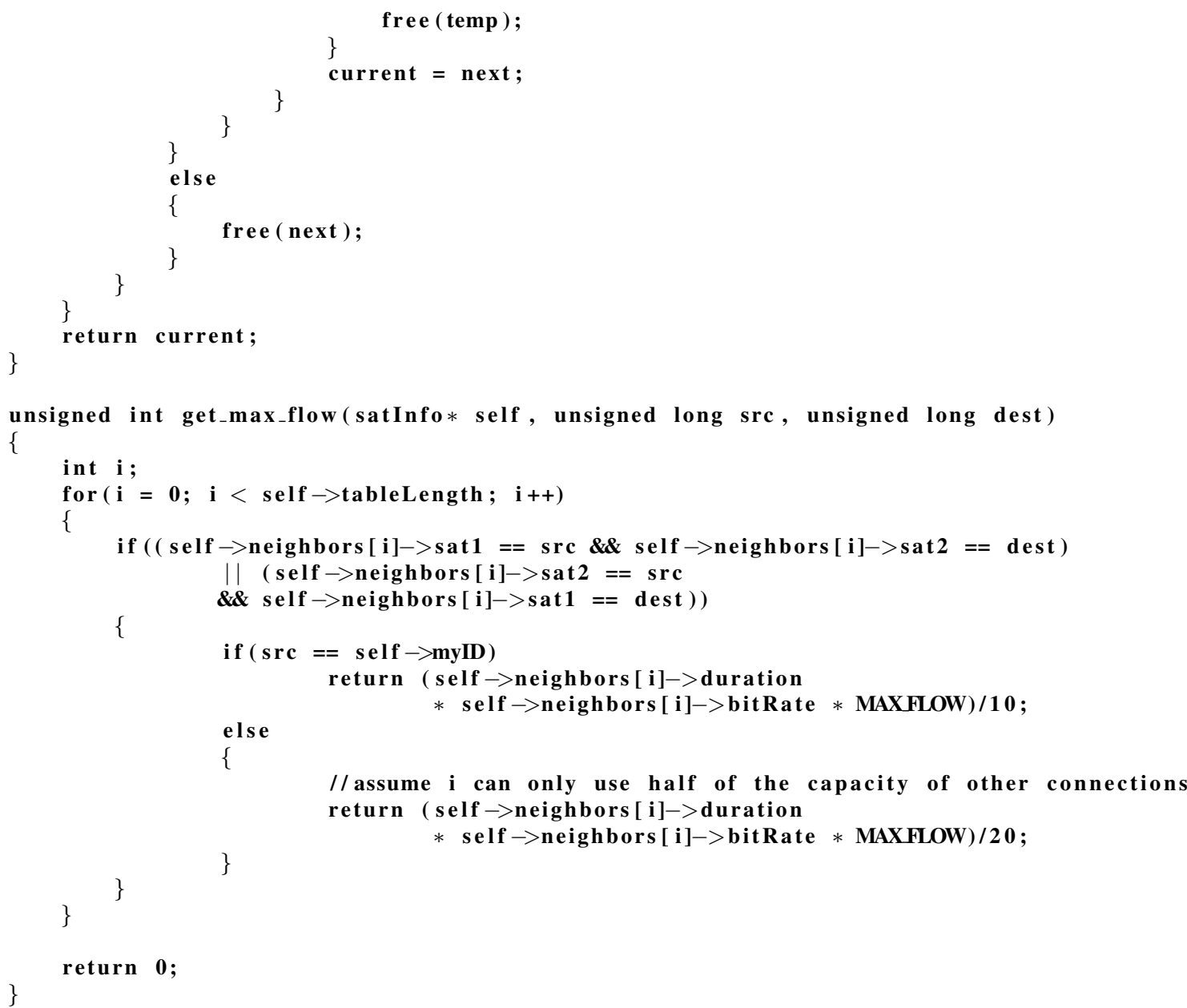

